JOURNAL OF THE

AMERICAN MATHEMATICAL SOCIETY

Volume 19, Number 2, Pages 265-326

S 0894-0347(05)00507-2

Article electronically published on November 18, 2005

\title{
CONFIGURATIONS, BRAIDS, AND HOMOTOPY GROUPS
}

\author{
A. J. BERRICK, F. R. COHEN, Y. L. WONG, AND J. WU
}

\section{Contents}

1. Introduction

2. Projective geometry and a decomposition of $F\left(S^{2}, n\right)$

3. Simplicial structures on configurations

3.1. Crossed simplicial groups

3.2. Crossed simplicial groups induced by configurations

4. $\Delta$-groups on configurations

4.1. $\Delta$-groups

4.2. $\Delta$-groups induced by configurations

5. Proof of Theorem 1.4

6. Proofs of Theorems 1.1, 1.2 and 1.3

6.1. A simplicial group model for $\Omega S^{2}$

6.2. Proof of Theorem 1.1

6.3. Artin's braids

6.4. Proof of Theorem 1.2

6.5. Proof of Theorem 1.3

6.6. Comparison of differentials

7. Low-dimensional Brunnian braids

7.1. The Moore homotopy groups $\pi_{n}\left(\mathcal{F}\left(S^{2}\right)^{\pi_{1}}\right)$ for $n \leq 3$

7.2. The Brunnian groups $\operatorname{Brun}_{n}\left(S^{2}\right)$ for $n \leq 4$

7.3. The Brunnian groups $\operatorname{Brun}_{n}\left(D^{2}\right)$ for $n \leq 4$ and relations between

$\operatorname{Brun}_{n}\left(D^{2}\right)$ and $\operatorname{Brun}_{n}\left(S^{2}\right)$ in low-dimensional cases

7.4. The 5 - and 6-strand Brunnian braids

8. Remarks

8.1. Notation

8.2. Birman's problem

8.3. Linear representation of the braid groups

Received by the editors April 28, 2003.

2000 Mathematics Subject Classification. Primary 20F36, 55Q40, 55U10; Secondary 20F12, 20F14, 57M50.

Key words and phrases. Braid group, Brunnian braid, configuration space, crossed simplicial group, Moore complex, homotopy groups of spheres.

Research of the first, third, and last authors is supported in part by the Academic Research Fund of the National University of Singapore R-146-000-048-112 and R-146-000-049-112.

The second author is partially supported by the US National Science Foundation grant DMS 0072173 and CNRS-NSF grant 17149.

(C)2005 American Mathematical Society Reverts to public domain 28 years from publication 
8.4. Artin's representation

8.5. Homotopy groups of spheres

8.6. Brunnian braids over $D^{2}$

Acknowledgments

References

\section{INTRODUCTION}

This paper introduces connections between the topology of configuration spaces and certain objects of a simplicial nature described below. These connections ultimately lead to geometric descriptions of elements of the homotopy groups of spheres in terms of special kinds of braids.

Let

$$
F(M, n)=\left\{\left(x_{0}, \ldots, x_{n-1}\right) \in M \times \cdots \times M \mid x_{i} \neq x_{j} \text { for } i \neq j\right\}
$$

be the $n$-th ordered configuration space of a space $M$. Let

$$
d_{i}: \pi_{1}(F(M, n+1)) \rightarrow \pi_{1}(F(M, n))
$$

be the group homomorphism $\pi_{1}\left(p_{i}\right)$ induced by the coordinate projection

$$
p_{i}: F(M, n+1) \rightarrow F(M, n), \quad p_{i}\left(x_{0}, \ldots, x_{n}\right)=\left(x_{0}, \ldots, x_{i-1}, x_{i+1}, \ldots, x_{n}\right),
$$

for $0 \leq i \leq n$, and suppose, for example, that $M$ is a cell complex of dimension at least 1, such that each $F(M, k)$ is path-connected. Then the sequence of groups

$$
\mathcal{F}(M)^{\pi_{1}}=\left\{\pi_{1}(F(M, n+1))\right\}_{n \geq 0}
$$

forms a $\Delta$-group; that is, the homomorphism $d_{i}$ satisfies the relation $d_{j} d_{i}=d_{i} d_{j+1}$ for $i \leq j$.

A detailed development of $\Delta$-groups is given in Section 4. A $\Delta$-set can be regarded as a simplicial set without degeneracies; thus the only operations are faces. A $\Delta$-group $\mathcal{G}=\left\{G_{n}\right\}_{n \geq 0}$ is a $\Delta$-set with the additional properties that each $G_{n}$ is a group and all faces are group homomorphisms. Let $\mathcal{X}$ be a $\Delta$-set and let $\mathbb{Z}(\mathcal{X})$ be the free abelian group generated (dimension-wise) by $\mathcal{X}$. Then $\mathbb{Z}(\mathcal{X})$ is a $\Delta$-group. Recall that the homology of $\mathcal{X}$ is obtained by taking the derived functor on $\mathbb{Z}(\mathcal{X})$. Namely, $\mathbb{Z}(\mathcal{X})$ is naturally a chain complex, and the homology of $\mathcal{X}$ is by definition the homology of the chain complex $\mathbb{Z}(\mathcal{X})$.

For a general (possibly noncommutative) $\Delta$-group $\mathcal{G}$, there is a similar derived functor described as follows. Define the Moore complex by

$$
N_{n} \mathcal{G}=\bigcap_{i \geq 1} \operatorname{Ker}\left(d_{i}: G_{n} \rightarrow G_{n-1}\right) .
$$

The homomorphism $d_{0}: G_{n} \rightarrow G_{n-1}$ induces a homomorphism $d_{0}: N_{n} \mathcal{G} \rightarrow N_{n-1} \mathcal{G}$ so as to make $N \mathcal{G}=\left\{N_{n} \mathcal{G}, d_{0}\right\}_{n \geq 0}$ a "chain complex" (of possibly noncommutative groups); that is, the composite

$$
d_{0} \circ d_{0}: N_{n+1} \mathcal{G} \rightarrow N_{n} \mathcal{G} \rightarrow N_{n-1} \mathcal{G}
$$

is the trivial map for any $n$. The set of left $\operatorname{cosets} \pi_{n}(\mathcal{G})$, which is not necessarily a group, is defined to be

$$
\pi_{n}(\mathcal{G})=\operatorname{Ker}\left(d_{0}: N_{n} \mathcal{G} \rightarrow N_{n-1} \mathcal{G}\right) / d_{0}\left(N_{n+1} \mathcal{G}\right) .
$$


Although the set of left cosets $\pi_{n}(\mathcal{G})$ need not be a group in general, it turns out that $\pi_{n}(\mathcal{G})$ is a group in many natural cases. (See Subsection 4.1 for details.)

Recall that for a surface $M$ the group $\pi_{1}(F(M, n))$ is called the $n$-strand pure braid group over $M$. The purpose of this paper is to investigate the $\Delta$-structure on $\mathcal{F}(M)^{\pi_{1}}$. As consequences, several connections between braid groups and the homotopy groups of $S^{2}$ are given. The first result concerns

$$
\mathcal{F}\left(S^{2}\right)^{\pi_{1}}=\left\{\pi_{1}\left(F\left(S^{2}, n+1\right)\right)\right\}_{n \geq 0},
$$

the sequence of pure braid groups over $S^{2}$ with faces described above.

Theorem 1.1. Let $\mathcal{F}\left(S^{2}\right)^{\pi_{1}}$ be the $\Delta$-group defined above. Then for each $n \geq 1$, $\pi_{n}\left(\mathcal{F}\left(S^{2}\right)^{\pi_{1}}\right)$ is a group, and there is an isomorphism of groups

$$
\pi_{n}\left(\mathcal{F}\left(S^{2}\right)^{\pi_{1}}\right) \cong \pi_{n}\left(S^{2}\right)
$$

for $n \geq 4$.

Low-dimensional specific computations of the groups $\pi_{n}\left(\mathcal{F}\left(S^{2}\right)^{\pi_{1}}\right)$ for $n \leq 3$ are described in Subsection [7.1. One particular point is that $\pi_{3}\left(\mathcal{F}\left(S^{2}\right)^{\pi_{1}}\right)$ is a noncommutative group (with center isomorphic to $\pi_{3}\left(S^{2}\right) \cong \mathbb{Z}$ ). This information shows that $\mathcal{F}\left(S^{2}\right)^{\pi_{1}}$ does not have a simplicial group structure. On the other hand, it emerges that the $\Delta$-group $\mathcal{F}(M)^{\pi_{1}}$ is close to being a simplicial group (see Subsection 4.2 for details). The isomorphisms in Theorem 1.1 are obtained by establishing a connection, described in Subsection 6.1, between the $\Delta$-group $\mathcal{F}\left(S^{2}\right)^{\pi_{1}}$ and Milnor's free group construction $F\left[S^{1}\right]$ with geometric realization homotopy equivalent to $\Omega S^{2}$.

Theorem 1.1 describes each (higher) homotopy group of the 2-sphere as a derived group of the pure braid groups over $S^{2}$. In other words, $\pi_{n}\left(S^{2}\right)$ is a certain "canonical" subquotient of the pure braid group $\mathcal{F}\left(S^{2}\right)_{n}^{\pi_{1}}=\pi_{1}\left(F\left(S^{2}, n+1\right)\right)$ for $n \geq 4$. In the isomorphism of the theorem, on the right, the groups $\pi_{n}\left(S^{2}\right)(n \geq 4)$ are all known to be finite in case $n \geq 4$. Next, recall the classical isomorphism $\pi_{n}\left(S^{2}\right) \cong \pi_{n}\left(S^{3}\right)$ for $n \geq 3$ that follows at once from the Hopf fibration. In addition, the following classical result due to Serre can be found in Spanier's book 66, Corollary 9.7.12]: Let $n \geq 3$ be odd and $p$ prime. Then $\pi_{i}\left(S^{n}\right)$ and $\pi_{i-n+3}\left(S^{3}\right)$ have isomorphic $p$-primary components if $i<4 p+n-6$. Some important elements in higher stable homotopy groups of spheres are suspensions of elements in the homotopy groups of the 3-sphere originating from $\pi_{*}\left(S^{2}\right)$; see for instance 67.

For the groups on the left in Theorem 1.1, recall that the center $\mathcal{Z}\left(\mathcal{F}\left(S^{2}\right)_{n}^{\pi_{1}}\right)$ is $\mathbb{Z} / 2$ for $n \geq 2$, and $\mathcal{F}\left(S^{2}\right)_{n}^{\pi_{1}} / \mathcal{Z}\left(\mathcal{F}\left(S^{2}\right)_{n}^{\pi_{1}}\right)$ is the pure mapping class group on the 2-sphere with $n+1$ punctured points; see [6]. Theorem 1.1 also describes the (higher) homotopy groups of the 2 -sphere as derived groups of pure mapping class groups.

Observe that the symmetric group $S_{n}$ acts on $F(M, n)$ by permuting the $n$ coordinates. Let $B(M, n)$ denote the quotient space $S_{n} \backslash F(M, n)$. An element in $\pi_{1}(B(M, n))$ is called a braid of $n$ strings over $M$ and $\pi_{1}(B(M, n))$ is known as the $n$-strand braid group over $M$. Since $S_{n}$ acts freely on $F(M, n)$, the quotient map $F(M, n) \rightarrow B(M, n)$ is a covering. Thus any loop in $B(M, n)$ admits a unique path-lifting to $F(M, n)$ with a specified basepoint; in other words, the elements in $\pi_{1}(B(M, n))$ are in one-to-one correspondence with the geometric braids of $n$ strings on $M$. (See Subsection 3.2 for details.) A braid of $n$ strings is called Brunnian if it becomes a trivial braid after removing any one of its strings. For instance, the 
well-known Borromean rings comprise the link obtained by closing up a Brunnian braid of three strings over $D^{2}$.

Let $\operatorname{Brun}_{n}(M)$ be the set of Brunnian braids of $n$-strings on $M$. Then $\operatorname{Brun}_{n}(M)$ is a group under composition of braids. Observe that the canonical inclusion of the disk into the sphere $f: D^{2} \hookrightarrow S^{2}$ (as northern hemisphere) induces a group homomorphism

$$
f_{*}: \operatorname{Brun}_{n}\left(D^{2}\right) \rightarrow \operatorname{Brun}_{n}\left(S^{2}\right) .
$$

As noted in Proposition 4.2.5, the groups $\operatorname{Brun}_{n}(M)$ are free for $n \geq 4$ when $M$ is a surface. Moreover, it follows from the next result (and low-dimensional information in Section 7) that the groups $\operatorname{Brun}_{n}(M)$ occurring in the theorem are all of infinite rank.

Theorem 1.2. There is an exact sequence of groups

$$
1 \longrightarrow \operatorname{Brun}_{n+1}\left(S^{2}\right) \longrightarrow \operatorname{Brun}_{n}\left(D^{2}\right) \stackrel{f_{*}}{\longrightarrow} \operatorname{Brun}_{n}\left(S^{2}\right) \longrightarrow \pi_{n-1}\left(S^{2}\right) \longrightarrow 1
$$

for $n \geq 5$.

The methods for proving this theorem are to describe the Brunnian braids $\operatorname{Brun}_{n}(M)$ as the cycles in the $\Delta$-group $\mathcal{F}(M)^{\pi_{1}}$ and to analyze the short exact sequence of $\Delta$-groups associated to the epimorphism $\mathcal{F}\left(D^{2}\right)^{\pi_{1}} \rightarrow \mathcal{F}\left(S^{2}\right)^{\pi_{1}}$. The analysis for low-dimensional cases where $n \leq 4$ is given in Subsection 7.3.

Theorem 1.2 reveals that any nontrivial element in the (higher) homotopy groups of the 2-sphere can be represented by a Brunnian braid over $S^{2}$ that is not Brunnian over $D^{2}$. Roughly speaking, the "difference" between the Brunnian braids over $S^{2}$ and those over $D^{2}$ is exactly measured by the homotopy groups. By means of canonical relations between braids and the mapping classes, this opens up the possibility of studying the homotopy groups of $S^{2}$ by geometry on braids or mapping class groups.

There is also a presentation of the homotopy groups of the 2-sphere solely in terms of Brunnian braids over the disk. First consider an operation $\tilde{\partial}: B_{n+1} \rightarrow$ $B_{n}:=\pi_{1}\left(B\left(D^{2}, n\right)\right)$ as follows. Let $\delta: F(\mathbb{C}, n+1) \longrightarrow F(\mathbb{C}, n)$ be the map defined by

$$
\delta\left(z_{0}, z_{1}, \ldots, z_{n}\right)=\left(\frac{1}{\bar{z}_{1}-\bar{z}_{0}}, \frac{1}{\bar{z}_{2}-\bar{z}_{0}}, \ldots, \frac{1}{\bar{z}_{n}-\bar{z}_{0}}\right),
$$

corresponding geometrically to inversion in $\mathbb{C}$ with respect to the unit circle centered at $z_{0}$. In Subsection 6.5 on fundamental groupoids, it is shown that $\delta$ induces a function $\tilde{\partial}: B_{n+1} \rightarrow B_{n}$ that restricts to a group homomorphism from $P_{n+1}$ to $P_{n}:=\pi_{1}\left(F\left(D^{2}, n\right)\right)$ and from $\operatorname{Brun}_{n+1}\left(D^{2}\right)$ to $\operatorname{Brun}_{n}\left(D^{2}\right)$. (However, the function $\tilde{\partial}: B_{n+1} \rightarrow B_{n}$ is not itself a group homomorphism.) Notice that there is a homomorphism $\chi: B_{n} \longrightarrow B_{n}$ that sends each standard generator to its inverse, because such a homomorphism preserves the relations for the braid group. Likewise $\chi$ restricts to a group homomorphism from $P_{n}$ to $P_{n}$ and from $\operatorname{Brun}_{n}\left(D^{2}\right)$ to $\operatorname{Brun}_{n}\left(D^{2}\right)$. Composing $\chi$ with $\tilde{\partial}$ gives a homomorphism $\partial$ on $\operatorname{Brun}_{n+1}\left(D^{2}\right)$ that maps into $\operatorname{Brun}_{n}\left(D^{2}\right)$ and has the further property that $\partial \circ \partial$ is trivial. There is the associated chain complex $\left(\operatorname{Brun}\left(D^{2}\right), \partial\right)$ in the sense of the Moore complex as described above for nonabelian groups:

$$
\cdots \rightarrow \operatorname{Brun}_{n+1}\left(D^{2}\right) \stackrel{\partial}{\rightarrow} \operatorname{Brun}_{n}\left(D^{2}\right) \stackrel{\partial}{\rightarrow} \operatorname{Brun}_{n-1}\left(D^{2}\right) \rightarrow \cdots \rightarrow \operatorname{Brun}_{1}\left(D^{2}\right)=1 .
$$


Theorem 1.3. For all $n$ there is an isomorphism of groups

$$
H_{n}\left(\operatorname{Brun}\left(D^{2}\right)\right) \cong \pi_{n}\left(S^{2}\right) \text {. }
$$

The proof arises as a construction of a $\Delta$-group $\Gamma$ that can be regarded as a model for $S^{2}$, in that $\pi_{n}(\Gamma) \cong \pi_{n}\left(S^{2}\right)$ for all $n$. Since $S^{2}$ is not an $H$-space, it is not homotopy equivalent to the geometric realization of a simplicial group. Hence the model above for $S^{2}$ can be seen as a counterpart of the usual construction of a simplicial group model for an $H$-space.

Theorems 1.1 and 1.2 are obtained by considering the special, but informative, cases given by $M=D^{2}$ or $S^{2}$. There are related simplicial groups associated to many configuration spaces as follows, where a metric space with a steady flow is used. Let $(M, d)$ be a metric space with basepoint $w$ and let $\mathbb{R}^{+}=[0, \infty)$. A steady flow over $M$ is a (continuous) map

$$
\theta: \mathbb{R}^{+} \times M \rightarrow M
$$

such that

(1) for any $x \in M, \theta(0, x)=x$ and for $t$,

$$
0<d(\theta(t, x), x) \leq t
$$

(2) $\left.\theta\right|_{\mathbb{R}^{+} \times\{w\}}: \mathbb{R}^{+} \times\{w\} \rightarrow M$ is one-to-one;

(3) there exists a function $\epsilon: \mathbb{R}^{+} \rightarrow(0,+\infty), t \mapsto \epsilon_{t}$, such that

$$
\theta\left(\left[0, \epsilon_{t}\right) \times\{\theta(t, w)\}\right) \subseteq \theta([t, \infty) \times\{w\})
$$

for any $t \in \mathbb{R}^{+}$.

Features of steady flows are developed in Section 3 below. For instance, if $M$ is a differentiable manifold with a nonvanishing vector field, then $M$ has a steady flow; see Proposition 3.2.4.

Let $[A, X]$ denote the set of pointed homotopy classes of maps. Recall that the pointed homotopy classes of maps $[A, X]$ is a group if $A$ is a cogroup space. In particular, $[A, F(M, n)]$ is a group if $A$ is a cogroup space. Moreover, the face and degeneracy operations induced by the pointed maps for a metric space $M$ with a steady flow satisfy the simplicial identities up to pointed homotopies.

Let $M$ and $A$ be pointed spaces. Define $\Gamma_{*}(A, M)$ with

$$
\Gamma_{n}(A, M)=[A, F(M, n+1)]
$$

for $n \geq 0$. A space $M$ is said to have a good basepoint $w$ if there is a continuous injection $\tilde{\theta}: \mathbb{R}^{+} \rightarrow M$ with $\tilde{\theta}(0)=w$. Thus in particular a metric space with a steady flow has a good basepoint.

Theorem 1.4. Let $M$ be a space with a good basepoint and let $A$ be a pointed space. Then the following hold.

(i) The projection maps specified by Equation (9) in Section 5 give $\Gamma_{*}(A, M)$ the structure of a $\Delta$-set.

(ii) If $M$ is a metric space with a steady flow, then the degeneracy maps specified in Equation (10) of Section 5 give $\Gamma_{*}(A, M)$ the structure of a simplicial set. Furthermore, if $A$ is a suspension (or more generally, a cogroup), then $\Gamma_{*}(A, M)$ is a simplicial group.

Remark. In case $M=\mathbb{C}^{k}$ for $k>1$, and $F\left(\mathbb{C}^{k}, n+1\right)$ is localized at the rational numbers, then $\left[\Sigma \Omega S^{2}, F\left(\mathbb{C}^{k}, n+1\right)_{\mathbb{Q}}\right]$ is isomorphic to the Malceev completion of the $(n+1)$ st pure braid group $P_{n+1}$ (see [61]). 
Some historical remarks concerning simplicial groups and this paper are given next. As a combinatorial tool for studying homotopy theory, simplicial groups were first studied by J. C. Moore [56]. The classical Moore theorem states that $\pi_{*}(|\mathcal{G}|) \cong H_{*}(N \mathcal{G})$, where $|\mathcal{G}|$ is the geometric realization of $\mathcal{G}$ and $N \mathcal{G}$ is the Moore chain complex of $\mathcal{G}$ described as above. Milnor 54 then proved that any loop space is (weakly) homotopy equivalent to a geometric realization of a simplicial group, and so, theoretically speaking, the homotopy groups of any space can be determined as the homology of a Moore chain complex. It is possible that two simplicial groups with the same homotopy type have sharply different group structures. Simplicial group models for loop spaces have been studied by many people; see for instance 3 , 13, 17, 40, 55, 56, 59, 65, 69. Different simplicial group models for the same loop space may give different homotopy information. For example, the classical Adams spectral sequence arises from the mod $p$ descending central series of Kan's $G$-construction on reduced simplicial sets, [10, 11, 21. On the other hand, one could have a perfect simplicial group model (that is, the abelianization is the trivial group) for certain loop spaces by using Carlsson's construction [13, 69]. For this model, the descending central series will not give any information as the groups are perfect, but word filtration provides different information.

Recently, by using Milnor's free group construction on the circle [55, which is a simplicial group model for $\Omega S^{2}$, it was proved that the general homotopy group $\pi_{n}\left(S^{2}\right)$ is isomorphic to the center of a combinatorially given group $G_{n}$ with $n$ generators and certain systematic relations 70 , Theorem 1.4]. Moreover, the Artin braid group $B_{n}$ acts on the group $G_{n}$, and the homotopy group $\pi_{n}\left(S^{2}\right)$ is given by the fixed set of the pure braid group $P_{n}$ action on $G_{n}$ [71, Theorem 1.2].

It was shown in [18] that Milnor's free group construction for the circle $F\left[S^{1}\right]$ admits a faithful representation into a simplicial group arising from Artin's pure braid groups with a simplicial structure analogous to that above. That the representation is faithful arises from properties of Yang-Baxter Lie algebras. Since one of the definitions of braid groups is as the fundamental groups of unordered configuration spaces, the relations between Artin braids and homotopy groups given in 18, 71 are extended in this article by studying connections between the topology of configuration spaces and variations of simplicial groups.

The methods in this article for constructing simplicial and $\Delta$-group models depart from traditional group-theoretical constructions. In particular, here grouptheoretic features of the simplicial and $\Delta$-structures on configuration spaces are considered intrinsically. This approach differs from classical approaches which address functors from sets to groups for obtaining simplicial group models.

Theorems 1.1 1.4 suggest that further simplicial group models may be obtained by systematically studying important mathematical objects in different areas. The simplicial groups that arise may give connections between homotopy theory and other areas; and the homotopy groups may describe certain global invariants in a novel yet canonical way. For instance, Theorem 1.2 describes the difference between the Brunnian braids over the sphere and those over the disk.

Configuration spaces were introduced mathematically in 1962 by E. Fadell and L. Neuwirth 26] and have been studied in various areas of mathematics and physics. In low-dimensional topology, configuration spaces form one of the basic tools for studying links and knots; for instance, for finding defining relations in the braid groups of surfaces [6] and for finding invariants of knots and links, see for instance [8, 
9, 14, 43, 44, 45, 46. In knot theory, a Brunnian link is defined to be a nontrivial link such that every proper sublink is trivial [60, page. 67]. A Brunnian braid was called a decomposable braid in [48] and a smooth braid in 39. Clearly a link obtained by closing up a Brunnian braid is a Brunnian link, but there are Brunnian links, for example the Whitehead link, that cannot be obtained by closing up a Brunnian braid. In addition, a result of Mangum and Stanford is that Brunnian links are determined by their complements [51. Additional discussions concerning the geometric properties of Brunnian links and Brunnian mapping classes are given in [51, 68.

More discussion on Brunnian links can be found in 24, 58, while some applications of Brunnian links to bio-organic chemistry occur in [49. In the terminology here, a Brunnian cycle means a Moore cycle in a $\Delta$ - or simplicial group (or set). Theorems 1.1 and 1.2 give new information on Brunnian braids and this information is related to the homotopy groups. There are some problems arising from the representations of the braid groups that are equivalent to finding a free basis for Moore cycles (see Subsection 8 for details).

In addition to low-dimensional topology, the braid groups and mapping class groups of course have wide use in many other areas such as algebraic geometry, number theory and quantum mechanics. The homotopy groups of spheres thus arise as new derived groups of these useful groups, and might thereby admit applications in many areas. On the other hand, Theorems 1.1 and 1.2 also suggest that it might be possible to study the homotopy groups of spheres by using methods in different areas of mathematics and physics. In algebraic geometry, observe that the sphere $S^{2}$ is homeomorphic to the projective space $\mathbb{C P}^{1}$. The space $F\left(\mathbb{C P}^{1}, n\right)$ is one of increasing importance in algebraic geometry.

There is a classical decomposition which has been used in several places:

$$
F\left(\mathbb{C P}^{1}, n\right) \approx F\left(\mathbb{C P}^{1}, 3\right) \times F(\mathbb{C}-\{0,1\}, n-3)
$$

for $n \geq 3$. This decomposition essentially derives from the classical "fundamental theorem of projective geometry". (See Section 2 for details.) Input from mathematical physics has recently spurred much interest in the compactifications of these spaces. Observe that the Brunnian braids over $S^{2}$ can be represented by certain loops in $F\left(\mathbb{C P}^{1}, n\right)$. It therefore seems possible that one could study the homotopy groups $\pi_{*}\left(S^{2}\right)$ by using methods in algebraic geometry prompted by physical connections. So far it is not clear whether the homotopy groups $\pi_{*}\left(S^{2}\right)$, as the derived groups of the fundamental groups of the spaces $F\left(\mathbb{C P}^{1}, n\right)$, provide new information to algebraic geometry or physics, but there are natural connections.

The homotopy groups $\pi_{*}\left(S^{2}\right)$ are known for $* \leq 64$; see [22]. Up to this range, by using Theorem 1.2, we are able to determine the cokernel of $f_{*}: \operatorname{Brun}_{n}\left(D^{2}\right) \rightarrow$ $\operatorname{Brun}_{n}\left(S^{2}\right)$. For instance, $\operatorname{Brun}_{5}\left(S^{2}\right) \bmod \operatorname{Brun}_{5}\left(D^{2}\right)$ is isomorphic to $\pi_{4}\left(S^{2}\right)=$ $\mathbb{Z} / 2$. The general homotopy groups $\pi_{*}\left(S^{2}\right)$ are unknown of course.

In this article, it will be assumed that the space $M$ has a good basepoint $w$, namely, there is a continuous injection $\tilde{\theta}: \mathbb{R}^{+} \rightarrow M$ with $\tilde{\theta}(0)=w$. The basepoint for $F(M, n+1)$ is $\left(w_{0}, \ldots, w_{n}\right)$, where $w_{0}$ is a good basepoint for $M$ and $w_{i}=\tilde{\theta}(i)$.

The article is organized as follows. Section 2 gives the decomposition of $F\left(\mathbb{C P}^{1}, n\right)$ and its connections to projective geometry. Simplicial structures of braids are given in Section 3 In Section 4 , the relationship between configuration spaces and $\Delta$ groups is given. Theorem 1.4 is proved in Section [5. The proofs of Theorems 1.1, 1.2 
and 1.3 are given in Section 6 followed by analysis of low-dimensional cases in Section 7, and miscellaneous remarks in Section 8 .

\section{Projective geometry and a Decomposition of $F\left(S^{2}, n\right)$}

Given a topological field $K$, recall that a hyperplane of the projective space

$$
K P^{m}=\left(K^{m+1}-\{0\}\right) / \mathrm{GL}_{1}(K)
$$

is the image under the projection $K^{m+1}-\{0\} \rightarrow K P^{m}$ of a subspace of $K^{m+1}$ of dimension $m$. Let $\operatorname{GP}\left(K P^{m}, r\right)$ be the configuration space of $r$ points in $K P^{m}$ in general position; in other words it is the subspace of $F\left(K P^{m}, r\right)$ such that no $m+1$ points lie on a hyperplane. In the particular case when $m=1$, this requirement is that the points in the projective line should be distinct. It follows that $\operatorname{GP}\left(K P^{1}, n\right)=F\left(K P^{1}, n\right)$. The action of $P \mathrm{GL}_{m+1}(K)$ on $K P^{m}$ extends diagonally, to give an action on $F\left(K P^{m}, r\right)$.

The classical "Fundamental Theorem of Projective Geometry" can be expressed in current language as follows.

Lemma 2.1. $P \mathrm{GL}_{m+1}(K)$ acts freely and transitively on $\mathrm{GP}\left(K P^{m}, m+2\right)$.

Proof. Freeness is proved in, for example, [34, (7.1.1)], and transitivity in, say, 42, $(2.22)]$.

It follows that the image of a fixed configuration q of $\operatorname{GP}\left(K P^{m}, m+2\right)$ determines a bijection from $P \mathrm{GL}_{m+1}(K)$ to $\mathrm{GP}\left(K P^{m}, m+2\right)$. In fact, this bijection is easily seen to be a homeomorphism labelled $\Phi$.

Next, given any configuration $\mathbf{q} \in \operatorname{GP}\left(K P^{m}, q\right)$ with underlying set $Q \subseteq K P^{m}$, define the space $\operatorname{GP}\left(K P^{m}-Q, r\right)$ by requiring that $\mathbf{r} \in \operatorname{GP}\left(K P^{m}-Q, r\right)$ if $\mathbf{q} \cup \mathbf{r}$ (ordered union) represents a configuration in $\mathrm{GP}\left(K P^{m}, q+r\right)$.

Theorem 2.2. Let $\mathbf{q} \in \operatorname{GP}\left(K P^{m}, m+2\right)$ with underlying set $Q \subseteq K P^{m}$. Then for $n \geq 0$ there are homeomorphisms from $\mathrm{GP}\left(K P^{m}-Q, n\right) \times P \mathrm{GL}_{m+1}(K)$ to $\mathrm{GP}\left(K P^{m}-Q, n\right) \times \operatorname{GP}\left(K P^{m}, m+2\right)$ and to $\operatorname{GP}\left(K P^{m}, n+m+2\right)$.

Proof. The first homeomorphism is id $\times \Phi$. The second sends a point $(\mathbf{r}, \alpha)$ in $\mathrm{GP}\left(K P^{m}-Q, n\right) \times P \mathrm{GL}_{m+1}(K)$ to $\alpha \mathbf{q} \cup \alpha \mathbf{r}$.

Since $\operatorname{GP}\left(K P^{m}-Q, 0\right)$ is a one-point space, this result generalizes what was stated before. Another case, $m=n=1$, is also classical, because the punctured line $\operatorname{GP}\left(K P^{1}-\{0,1, \infty\}, 1\right)=K P^{1}-\{0,1, \infty\}$ is just the cross-ratio. More generally, that $F\left(K P^{1}, n+3\right)$ forms a principal $P \mathrm{GL}_{2}(K)$-bundle over $F\left(K P^{1}-\{0,1, \infty\}, n\right)$ is well known to algebraic geometers. The fact that the bundle is trivial is also recorded in [7.

Other specializations (see Corollary 2.3) exploit the homeomorphisms

$$
\mathbb{C} P^{1} \approx S^{2} \approx \mathbb{R}^{2} \cup\{\infty\} \text { and } \mathbb{R} P^{1} \approx S^{1} \approx \mathbb{R}^{1} \cup\{\infty\},
$$

and the well-known homotopy equivalences $P G L_{2}(\mathbb{C}) \simeq \mathbb{R P} P^{3} \simeq P G L_{3}(\mathbb{R})$ and $P \mathrm{GL}_{2}(\mathbb{R}) \simeq S^{1}$.

Corollary 2.3. The homeomorphisms of the theorem above give rise to the following homeomorphisms and homotopy equivalences:

(i) $F\left(S^{2}, n+3\right) \approx F\left(\mathbb{R}^{2}-\{0,1\}, n\right) \times F\left(S^{2}, 3\right) \simeq F\left(\mathbb{R}^{2}-\{0,1\}, n\right) \times \mathbb{R P}^{3}$;

(ii) $F\left(S^{1}, n+3\right) \approx F\left(\mathbb{R}^{1}-\{0,1\}, n\right) \times F\left(S^{1}, 3\right) \simeq F\left(\mathbb{R}^{1}-\{0,1\}, n\right) \times S^{1}$; 
(iii) $\mathrm{GP}\left(\mathbb{R P}^{2}, n+4\right) \simeq \mathrm{GP}\left(\mathbb{R P}^{2}-\Delta, n\right) \times \mathbb{R} \mathrm{P}^{3}$. (Here, $\Delta$ represents the standard coordinate simplex of four points in $\mathbb{R P}^{2}$.)

Remark. The decomposition in part (i) has been given in [7] and also [29, Theorem 2.1]. The decomposition in (ii) is a special case of a decomposition given by Fadell and Neuwirth [26].

The authors are grateful to B. Hassett and J. Morava for helpful communications concerning the Grothendieck-Mumford-Knudsen compactification $\overline{\mathcal{M}}_{0, n+3}(\mathbb{C})$ of $F\left(\mathbb{R}^{2}-\{0,1\}, n\right)$ and its significance in algebraic geometry and mathematical physics. This is the moduli space of marked stable algebraic curves of genus zero (having at worst double points, and at least three marked points on each irreducible component). It is a smooth variety of complex dimension $n$ whose (rational) homology has been shown to be isomorphic to its Chow ring and comprises finitedimensional vector spaces concentrated in even dimensions [4]. The computation results from an explicit factorization, as a product of blowups, of the extension to $\overline{\mathcal{M}}_{0, n+3}(\mathbb{C})$ of the natural inclusion of $F\left(\mathbb{R}^{2}-\{0,1\}, n\right)$ in $\left(S^{2}\right)^{n}$. More recently, the compactification $\overline{\mathcal{M}}_{0, n+3}(\mathbb{R})$ of $F\left(\mathbb{R}^{1}-\{0,1\}, n\right)$ has also been studied; it has been shown to be aspherical [23, although its homology is less well understood 25. For further work on compactifications and homology of configuration spaces of algebraic varieties, see [31].

\section{Simplicial Structures on CONFigurations}

3.1. Crossed simplicial groups. Let $\mathcal{O}$ be the category of finite ordered sets and ordered functions, where a function $f$ is ordered if $f(x) \leq f(y)$ when $x \leq y$. The category $\mathcal{O}$ has objects $[n]=\{0, \ldots, n\}$ for $n \geq 0$, and morphisms are generated by the face functions $d^{i}:[n-1] \rightarrow[n]$ (which misses $i$ ) and the degeneracy functions $s^{i}:[n+1] \rightarrow[n]$ (which hits $i$ twice) for $0 \leq i \leq n$. Recall that a simplicial object $\mathcal{X}$ over a category $\mathcal{C}$ is a contravariant functor from $\mathcal{O}$ to $\mathcal{C}$. In other words, $\mathcal{X}=\left\{X_{n}\right\}_{n \geq 0}$, where $X_{n}=\mathcal{X}([n])$. The face $d_{i}: X_{n} \rightarrow X_{n-1}$ is given by $d_{i}=\mathcal{X}\left(d^{i}\right)$ and the degeneracy $s_{i}: X_{n} \rightarrow X_{n+1}$ is given by $s_{i}=\mathcal{X}\left(s^{i}\right)$ for $0 \leq i \leq n$. The simplicial identities follow from the well-known formulas for functions $d^{i}$ and $s^{j}$ in the category $\mathcal{O}$. A simplicial object over sets (resp. monoids, groups, Lie algebras, spaces, etc.) is called a simplicial set (resp. monoid, group, Lie algebra, space, etc.). Standard references for the theory of simplicial objects are [21, 52 .

Example 3.1.1. Let $S_{n+1}$ denote the symmetric group of bijections of the symbols $0,1, \ldots, n$. Sometimes right actions of $S_{n}$ are used by requiring $i \cdot \sigma=\sigma^{-1}(i)$. Let $\mathcal{S}=\left\{S_{n+1}\right\}_{n \geq 0}$ be the sequence of symmetric groups of degree $n+1$. Then $\mathcal{S}$ is a simplicial set in the following way. The face $d_{i}: S_{n+1} \rightarrow S_{n}$ is uniquely determined by the commutative diagram

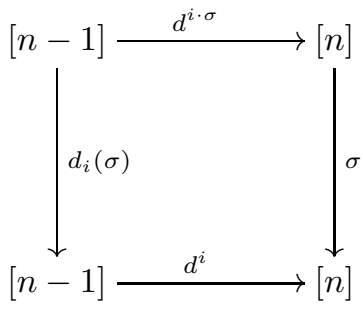


for any $\sigma \in S_{n+1}$; that is, $d_{i}(\sigma)=s^{i} \circ \sigma \circ d^{\sigma^{-1}(i)}$. The degeneracies $s_{i}: S_{n+1} \rightarrow S_{n+2}$ are determined uniquely by requiring

$$
s_{i}(\sigma)\left(\sigma^{-1}(i)\right)=i, \quad s_{i}(\sigma)\left(\sigma^{-1}(i)+1\right)=i+1
$$

and the diagram

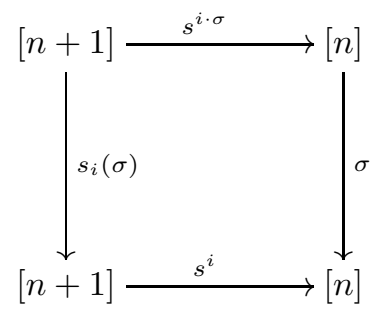

commutes; that is,

$$
s_{i}(\sigma)(k)= \begin{cases}\left(s^{i}\right)^{-1} \sigma s^{\sigma^{-1}(i)}(k) & k \neq \sigma^{-1}(i), \sigma^{-1}(i)+1 \\ i & k=\sigma^{-1}(i) \\ i+1 & k=\sigma^{-1}(i)+1\end{cases}
$$

Note that Equation 2 follows from the commutative diagrams 1 and 3 together with simplicial identities: $s_{1} s_{0}=s_{0} s_{0}$ for the case $n=0$ and the expression for $d_{k} s_{i}$, $k \neq i, i+1$, for the case $n \geq 1$.

Fiedorowicz and Loday defined crossed simplicial groups [30].

A crossed simplicial group is a simplicial set $\mathcal{G}=\left\{G_{n}\right\}_{n \geq 0}$ for which each $G_{n}$ is a group, together with a group homomorphism $\mu$ : $G_{n} \rightarrow S_{n+1}, g \mapsto \mu_{g}$ for each $n$, such that

(i) $\mu$ is a simplicial map, and

(ii) for $0 \leq i \leq n, d_{i}\left(g g^{\prime}\right)=d_{i}(g) d_{i \cdot \mu_{g}}\left(g^{\prime}\right)$ and $s_{i}\left(g g^{\prime}\right)=s_{i}(g) s_{i \cdot \mu_{g}}\left(g^{\prime}\right)$.

It is routine to see that this definition is equivalent to the characterization given in [30, Proposition 1.7]. The key example is that the simplicial set $\mathcal{S}$ with $\mu$ as the identity map is a crossed simplicial group. A simplicial group $\mathcal{G}$ becomes a crossed simplicial group when the homomorphism $\mu: G_{n} \rightarrow S_{n+1}$ is taken to be the trivial map. On the other hand, a crossed simplicial group need not be a simplicial group because the morphisms $d_{i}$ and $s_{i}$ need not be group homomorphisms.

A morphism $f: \mathcal{H} \rightarrow \mathcal{G}$ of crossed simplicial groups is a collection of group homomorphisms $f_{n}: H_{n} \rightarrow G_{n}$ such that $f=\left\{f_{n}\right\}$ is a simplicial map and also $\mu_{H}=\mu_{G} \circ f$. In particular, if each $f_{n}$ is an inclusion map, then $\mathcal{H}$ is a crossed simplicial subgroup of $\mathcal{G}$. From our definition, each crossed simplicial group $\mathcal{G}$ comes with a distinguished morphism $\mu: \mathcal{G} \rightarrow \mathcal{S}$. Then $\operatorname{Ker}(\mu: \mathcal{G} \rightarrow \mathcal{S})$ is a simplicial group and $\operatorname{Im}(\mu: \mathcal{G} \rightarrow \mathcal{S})$ is a crossed simplicial subgroup of $\mathcal{S}$. By determining the possible crossed simplicial subgroups of $\mathcal{S}$ one can thereby classify all crossed simplicial groups (cf. [30, Theorem 3.6]).

3.2. Crossed simplicial groups induced by configurations. Recall that the $n$-th ordered configuration space $F(M, n)$ of a space $M$ is defined by

$$
F(M, n)=\left\{\left(x_{0}, \ldots, x_{n-1}\right) \in M^{n} \mid x_{i} \neq x_{j} \text { for } i \neq j\right\}
$$


and has the topology of a subspace of the product space $M^{n}$. Here $S_{n}$ acts on $F(M, n)$ by permuting coordinates, that is,

$$
\begin{aligned}
\sigma \cdot\left(x_{0}, \ldots, x_{n-1}\right) & =\left(x_{0 \cdot \sigma}, \ldots, x_{(n-1) \cdot \sigma}\right) \\
& =\left(x_{\sigma^{-1}(0)}, \ldots, x_{\sigma^{-1}(n-1)}\right) .
\end{aligned}
$$

The orbit space $B(M, n)=S_{n} \backslash F(M, n)$ is called the $n$-th unordered configuration space. Let $\mathcal{F}(M)=\{F(M, n+1)\}_{n \geq 0}$ and let $\mathcal{B}(M)=\{B(M, n+1)\}_{n \geq 0}$. Define the faces $d_{i}: \mathcal{F}(M)_{n}=F(M, n+1) \rightarrow \mathcal{F}(M)_{n-1}=F(M, n)$ by

$$
d_{i}\left(x_{0}, \ldots, x_{n}\right)=\left(x_{0}, \ldots, x_{i-1}, x_{i+1}, \ldots, x_{n}\right)
$$

for $0 \leq i \leq n$. Additional assumptions concerning $M$ are required in order to construct analogues of "degeneracies" for $\mathcal{F}(M)$ as follows.

Definition 3.2.1. Let $(M, d)$ be a metric space with basepoint $w$ and let $\mathbb{R}^{+}$denote $[0, \infty)$. A steady flow over $M$ is a (continuous) map

$$
\theta: \mathbb{R}^{+} \times M \rightarrow M
$$

such that

(1) for any $x \in M, \theta(0, x)=x$ and for $t>0$,

$$
0<d(\theta(t, x), x) \leq t ;
$$

(2) $\left.\theta\right|_{\mathbb{R}^{+} \times\{w\}}: \mathbb{R}^{+} \times\{w\} \rightarrow M$ is one-to-one;

(3) there exists a function $\epsilon: \mathbb{R}^{+} \rightarrow(0,+\infty), t \mapsto \epsilon_{t}$, such that

$$
\theta\left(\left[0, \epsilon_{t}\right) \times\{\theta(t, w)\}\right) \subseteq \theta([t, \infty) \times\{w\})
$$

for any $t \in \mathbb{R}^{+}$.

Lemma 3.2.2. Let $\rho$ be a positive-valued real (not necessarily continuous) function on a paracompact topological space $M$. Then the following are equivalent.

(i) No convergent sequence $\left(y_{n}\right)$ in $M$ has $\rho\left(y_{n}\right) \rightarrow 0$.

(ii) Each $y \in M$ has $a \delta_{y}>0$ and neighborhood $V_{y}$ such that $\rho\left(V_{y}\right) \subseteq\left(\delta_{y}, \infty\right)$.

(iii) There is a continuous real-valued function $r$ on $M$ such that, for all $x$ in $M$,

$$
0<r(x) \leq \rho(x)
$$

Proof. Statements (i) and (ii) are equivalent by definition of convergence. Given (iii), any convergent sequence $y_{n} \rightarrow y$ with $\rho\left(y_{n}\right) \rightarrow 0$ also has $r\left(y_{n}\right) \rightarrow 0$. However, by continuity, $r\left(y_{n}\right) \rightarrow r(y)>0$.

Finally, to see that (ii) implies (iii), consider a partition of unity $\left\{\phi_{y}\right\}$ subordinate to the open covering $\left\{V_{y}\right\}_{y \in M}$ of $M$. Then put

$$
r(x)=\sum_{y \in M} \phi_{y}(x) \delta_{y} .
$$


The function $r(x)$ is continuous since each $\phi_{y}$ is, while

$$
r(x) \leq \max \left\{\delta_{y} \mid x \in \operatorname{supp} \phi_{y}\right\} \leq \sup \left\{\delta_{y} \mid x \in V_{y}\right\} \leq \rho(x) .
$$

Lemma 3.2.3. The above definition is equivalent to that with (1) replaced by the condition:

$\left(1^{\prime}\right)$ For each $x \in M, \theta(t, x)=x$ if and only if $t=0$, and there exists $\kappa>0$ such that $\lim _{t \rightarrow 0+} d(\theta(t, x), x) t^{-\kappa}=0$.

Proof. Clearly, if (1) holds, then choose $\kappa=\frac{1}{2}$, since

$$
d(\theta(t, x), x) t^{-1 / 2} \leq t^{1 / 2} .
$$

In the other direction, assume $\left(1^{\prime}\right)$ and the other two conditions for

$$
\theta^{\prime}: \mathbb{R}^{+} \times M \rightarrow M
$$

We first show how we may suppose that $\kappa$ is a continuous function of $x$. For each $x \in M$ let

$$
\kappa_{x}:=\sup \left\{\kappa \mid \lim _{t \rightarrow 0+} d\left(\theta^{\prime}(t, x), x\right) t^{-\kappa}=0\right\}>0 .
$$

Observe that for all $\kappa<\kappa_{x}$, it follows that $\lim _{t \rightarrow 0+} d\left(\theta^{\prime}(t, x), x\right) t^{-\kappa}=0$. In order to deny the possibility that $M$ contains a convergent sequence $y_{n} \rightarrow y$ with $\kappa_{y_{n}} \rightarrow 0$, fix $\kappa \in\left(0, \kappa_{y}\right)$. By eliminating terms as necessary from such a sequence, we may assume that, for all $n, \kappa_{y_{n}}<\kappa / 2$, so that there exists $u_{n} \in(0,1 / n)$ with

$$
d\left(\theta^{\prime}\left(u_{n}, y_{n}\right), y_{n}\right) u_{n}^{-\kappa / 2}>1 \text {. }
$$

On the other hand, $d\left(\theta^{\prime}(1 / n, y), y\right)(1 / n)^{-\kappa / 2} \rightarrow 0$ as $n \rightarrow \infty$. Since in $\mathbb{R}^{+} \times M$ both the sequences $\left(u_{n}, y_{n}\right)$ and $(1 / n, y)$ converge to $(0, y)$, the sequence

$$
\left(t_{m}, x_{m}\right)= \begin{cases}\left(u_{n}, y_{n}\right) & m=2 n+1, \\ (1 / n, y) & m=2 n,\end{cases}
$$

is a Cauchy sequence with the contradictory property that its image under the continuous function

$$
(t, x) \longmapsto d\left(\theta^{\prime}(t, x), x\right) t^{-\kappa / 2}
$$

is not Cauchy. Since metric spaces are paracompact (Michael's theorem), it follows from the preceding lemma that we may indeed suppose that $\kappa$ is a continuous function of $x$.

Now the function $f: \mathbb{R}^{+} \times M \rightarrow \mathbb{R}^{+}$defined by

$$
(t, x) \longmapsto \begin{cases}d\left(\theta^{\prime}(t, x), x\right) t^{-\kappa} & t>0, \\ 0 & t=0,\end{cases}
$$

is continuous. It follows that for all $y$ in $M$ the open set $f^{-1}([0,1))$ is a neighborhood of $(0, y)$ in $\mathbb{R}^{+} \times M$ and so contains some neighborhood of the form given by $[0, \delta(y)) \times B_{\delta(y)}(y)$. Now the function defined by

$$
\rho(x)=\sup \left\{t \mid[0, t) \times\{x\} \subseteq f^{-1}([0,1))\right\}
$$

evidently satisfies (ii) of the preceding lemma. Thus there is a continuous function $r: M \rightarrow(0, \pi / 2)$ such that for all $x$ and all $s \in[0, r(x)), f(s, x)<1$.

So define

$$
\theta: \mathbb{R}^{+} \times M \rightarrow M, \quad \theta(t, x)=\theta^{\prime}(s(t, x), x)
$$


where

$$
s(t, x):=\frac{2 r(x)}{\pi}\left(\frac{2}{\pi} \arctan t\right)^{1 / \kappa}<r(x) .
$$

Evidently condition (2) holds for $\theta$ just as for $\theta^{\prime}$, while condition (3) also holds since, for each $x$ in $M, s$ is a bijective function of $t$. Finally, for condition (1), because, for all $x \in M, f(s, x)<1$, it follows that

$$
d(\theta(t, x), x) \leq s^{\kappa}=\left(\frac{2 r}{\pi}\right)^{\kappa}\left(\frac{2}{\pi} \arctan t\right) \leq t .
$$

Proposition 3.2.4. If a differentiable manifold $M$ admits a (continuous) nonvanishing vector field, then there exists a steady flow over $M$.

Proof. First perturb the given continuous vector field to a $C^{1}$ nonvanishing vector field (by, for example, using standard results [12](II.11.9) to smooth the lifting of the classifying map for the spherical tangent bundle, so as to obtain a smooth section of a sphere bundle smoothly equivalent to the spherical tangent bundle). Then there exists a completely integrable $C^{1}$ vector field [35, p.155], so that its flow is also $C^{1}$, and has domain the whole of $\mathbb{R} \times M$. By differentiability of the flow, $\lim _{t \rightarrow 0+} d(\theta(t, x), x) t^{-\kappa}=0$ whenever $\kappa<1$. Conditions (2) and (3) may be attained by a suitable rescaling of the $t$ argument of $\theta$ in a neighborhood of $w$, as in the proof of the lemma.

Lemma 3.2.5. 35, (5.2)] If $M$ is a compact, connected differentiable manifold which either has nonempty boundary, or is oriented and has zero Euler characteristic, then $M$ admits a nonvanishing vector field.

Proposition 3.2.6. Let $M$ be a compact m-manifold without boundary. If $M$ is oriented over a field $\mathbb{F}$ and has nonzero Euler characteristic over $\mathbb{F}$, then $M$ does not have a steady flow.

Proof. The assertion will be proved by contradiction. Suppose that $M$ has a steady flow $\theta$. Consider the map $s: M \rightarrow M^{2}, x \mapsto(x, \theta(1, x))$. Since $d(x, \theta(1, x))>0$, it must be that $x \neq \theta(1, x)$ and so $s$ is a well-defined map into $F(M, 2)$, given by $s: M \rightarrow F(M, 2)$. Let $d_{0}, d_{1}: F(M, 2) \rightarrow M$ denote the first and the second coordinate projections, respectively. Then $d_{0} s=\mathrm{id}_{M}$ and $d_{1} s \simeq \operatorname{id}_{M}$ by a homotopy given by

$$
M \times I \rightarrow M, \quad(x, t) \mapsto \theta(t, x) .
$$

Thus the composite $M \stackrel{s}{\rightarrow} F(M, 2) \stackrel{j}{\hookrightarrow} M^{2}$ is homotopic to the diagonal map $\Delta: M \rightarrow M^{2}$.

Simply write $H^{*}(X)$ and $H_{*}(X)$ for $H^{*}(X ; \mathbb{F})$ and $H_{*}(X ; \mathbb{F})$, respectively. Let $t_{M} \in H^{m}\left(M^{2}, F(M, 2)\right)$ be an orientation of $M$ and let $U_{M}$ be the image of $t_{M}$ in $H^{m}\left(M^{2}\right)$. Write $z \in H_{m}(M)$ for the fundamental class of $M$. Let $\left\{u_{j}\right\}_{j \in J}$ be a (homogeneous) basis for $H^{*}(M)$. Then $H^{*}(M)$ has a dual basis $\left\{v_{k}\right\}_{k \in J}$ of $\left\{u_{j}\right\}$ with respect to the Poincaré duality, namely

$$
\left\langle v_{j} \cup u_{k}, z\right\rangle=\delta_{j k},
$$

where $\langle$,$\rangle is the Kronecker product and \delta_{j k}$ is the Kronecker $\delta$ notation. By [66, Lemma 1, p. 347], there is a formula:

$$
U_{M}=\sum_{i \in J}(-1)^{m \operatorname{deg}\left(u_{i}\right)} u_{i} \times v_{i} .
$$


(Note. Since $\left\{v_{j}\right\}$ is chosen to be the Poincaré dual basis, the matrix $B$ in 66 , Lemma 1, p. 347] is the identity matrix.) Since the diagonal $\Delta: M \rightarrow M^{2}$ maps into $F(M, 2)$ up to homotopy, from the previous paragraph,

$$
\Delta^{*}\left(U_{M}\right)=0
$$

because $U_{M}$ is the image of $t_{M} \in H^{m}\left(M^{2}, F(M, 2)\right)$ in $H^{m}\left(M^{2}\right)$. Thus

$$
\begin{aligned}
0 & =\left\langle\Delta^{*}\left(U_{M}\right), z\right\rangle=\sum_{i \in J}(-1)^{m \operatorname{deg}\left(u_{i}\right)}\left\langle u_{i} \cup v_{i}, z\right\rangle \\
& =\sum_{i \in J}(-1)^{m \operatorname{deg}\left(u_{i}\right)+\operatorname{deg}\left(u_{i}\right) \operatorname{deg}\left(v_{i}\right)}\left\langle v_{i} \cup u_{i}, z\right\rangle \\
& =\sum_{i \in J}(-1)^{m \operatorname{deg}\left(u_{i}\right)+\operatorname{deg}\left(u_{i}\right)\left(m-\operatorname{deg}\left(u_{i}\right)\right)}=\sum_{i \in J}(-1)^{\operatorname{deg}\left(u_{i}\right)} .
\end{aligned}
$$

Since the right-hand side is the Euler characteristic, it contradicts that $M$ has nonzero Euler characteristic.

The next statement for differentiable manifolds follows from the above.

Corollary 3.2.7. Let $M$ be a compact, connected oriented differentiable manifold. Then the following statements are equivalent.

(1) $M$ has nonempty boundary, or has zero Euler characteristic.

(2) $M$ admits a nonvanishing vector field.

(3) There exists a steady flow over $M$.

For the rest of this section, assume that $M$ is a metric space with a steady flow $\theta$. Let $\zeta: F(M, n+1) \rightarrow \mathbb{R}^{+}$be the map defined by

$$
\zeta(\mathbf{x})=\zeta\left(x_{0}, \ldots, x_{n}\right)=\frac{1}{2} \min \left\{1, \epsilon_{i}, d\left(x_{i}, x_{j}\right) \mid 0 \leq i<j \leq n\right\},
$$

where $\epsilon_{i}=\epsilon(i)$ for the function $\epsilon$ in Definition 3.2.1. Observe that $\zeta$ factors through $B(M, n)$. Define the function

$$
s_{i}: F(M, n+1) \rightarrow M^{n+2}
$$

by the formula

$$
s_{i}\left(x_{0}, \ldots, x_{n}\right)=\left(x_{0}, \ldots, x_{i}, \theta\left(\zeta(\mathbf{x}), x_{i}\right), x_{i+1}, \ldots, x_{n}\right)
$$

for $0 \leq i \leq n$. Let $x_{i}^{\prime}=\theta\left(\zeta(\mathbf{x}), x_{i}\right)$. Observe that, by (2), (3) of Definition 3.2.1, $0<d\left(x_{i}^{\prime}, x_{i}\right)<\zeta(\mathbf{x})$. For any $j \neq i, d\left(x_{i}, x_{j}\right) \leq d\left(x_{i}, x_{i}^{\prime}\right)+d\left(x_{i}^{\prime}, x_{j}\right)$. Hence,

$$
d\left(x_{i}^{\prime}, x_{j}\right) \geq d\left(x_{i}, x_{j}\right)-d\left(x_{i}, x_{i}^{\prime}\right) \geq d\left(x_{i}, x_{j}\right)-\zeta(x)>0 .
$$

Thus $x_{i}^{\prime}$ is distinct from $x_{0}, \ldots, x_{n}$, and so $s_{i}$ is a well-defined map into $F(M, n+2)$ given by

$$
s_{i}: F(M, n+1) \rightarrow F(M, n+2) .
$$

One consequence is that $\mathcal{F}(M)^{\pi_{1}}=\left\{\pi_{1}(F(M, n+1))\right\}_{n \geq 0}$ is a simplicial group under the faces and the degeneracies induced by the maps $d_{i}$ and $s_{i}$ above, and moreover, that $\mathcal{B}(M)^{\pi_{1}}=\left\{\pi_{1}(B(M, n+1))\right\}_{n \geq 0}$ is a crossed simplicial group. To prove this, some terminology concerning the fundamental groupoid of $\mathcal{F}(M)$ is given next.

Let $\mathbf{p}=\left(p_{0}, \ldots, p_{n}\right)$ and $\mathbf{q}=\left(q_{0}, \ldots, q_{n}\right)$ be two configurations in $F(M, n+1)$ and let $\lambda$ be a path in $F(M, n+1)$ from $\mathbf{p}$ to $\mathbf{q}$. Then $\lambda=\left(\lambda^{0}, \ldots, \lambda^{n}\right)$ is a sequence 
of paths in $M$ such that

(1) $\lambda^{i}(0)=p_{i}$ for $0 \leq i \leq n$;

(2) $\lambda^{i}(1)=q_{i}$ for $0 \leq i \leq n$;

(3) $\lambda^{i}(t) \neq \lambda^{j}(t)$ for $0 \leq i<j \leq n$ and $0 \leq t \leq 1$.

Conversely, any sequence of paths $\lambda=\left(\lambda^{0}, \ldots, \lambda^{n}\right)$ in $M$ such that the conditions above hold corresponds to a path from $\mathbf{p}$ to $\mathbf{q}$ in $F(M, n+1)$. Now let $\Lambda$ be a path homotopy from $\mathbf{p}$ to $\mathbf{q}$ in $F(M, n+1)$; that is, $\Lambda$ is a map

$$
\Lambda: I \times I \rightarrow F(M, n+1)
$$

such that $\Lambda(s, 0)=\mathbf{p}, \Lambda(s, 1)=\mathbf{q}$ for $0 \leq s \leq 1$. This gives a sequence of path homotopies $\Lambda=\left(\Lambda^{0}, \ldots, \Lambda^{n}\right)$ from $\left(\Lambda_{0}^{0}, \ldots, \Lambda_{0}^{n}\right)$ to $\left(\Lambda_{1}^{0}, \ldots, \Lambda_{1}^{n}\right)$ in $M$ such that $\Lambda^{i}(s, t) \neq \Lambda^{j}(s, t)$ for $0 \leq i<j \leq n$ and $0 \leq s, t \leq n$. Conversely, any sequence of path homotopies which satisfies these conditions induces a path homotopy in $F(M, n+1)$.

Write

$$
[\lambda]=\left[\lambda^{0}, \ldots, \lambda^{n}\right]
$$

for the path homotopy class in $F(M, n+1)$ represented by $\lambda=\left(\lambda^{0}, \ldots, \lambda^{n}\right)$. The symmetric group $S_{n+1}$-action on $F(M, n+1)$ induces an $S_{n+1}$-action on the fundamental groupoid $\varpi(F(M, n+1))$, where $\sigma \cdot[\lambda]=[\sigma \cdot \lambda]$ with

$$
\sigma \cdot \lambda=\left(\lambda^{0 \cdot \sigma}, \ldots, \lambda^{n \cdot \sigma}\right) .
$$

The maps $d_{i}$ and $s_{i}$ induce morphisms $d_{i}: \varpi(F(M, n+1)) \rightarrow \varpi(F(M, n))$ and $s_{i}: \varpi(F(n+1)) \rightarrow \varpi(F(M, n+2))$ for $0 \leq i \leq n$. The associated notation is that $(f \lambda)(t)=f(\lambda(t))$ is a path from $f(\lambda(0))$ to $f(\lambda(1))$ for any map $f$ and path $\lambda$. We write $\operatorname{Mor}(\mathbf{p}, \mathbf{q})$ for the set of path homotopy classes from $\mathbf{p}$ to $\mathbf{q}$, whenever $\mathbf{p}, \mathbf{q}$ are configurations in $F(M, m)$.

Lemma 3.2.8. Let $\mathbf{p}$ and $\mathbf{q}$ be two configurations in $F(M, n+1)$. Then there are commutative diagrams

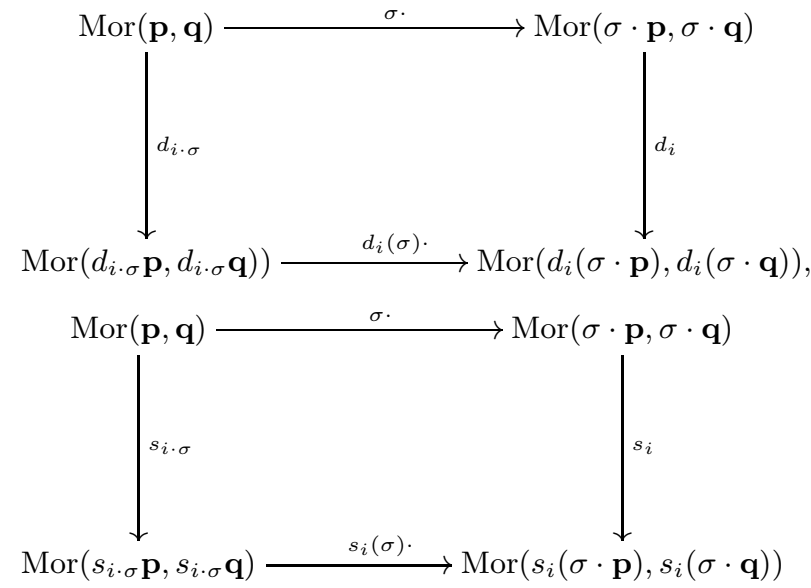

for $0 \leq i \leq n$. 
Proof. Let $\lambda$ be a path from $\mathbf{p}$ to $\mathbf{q}$. Then

$$
d_{i}(\sigma \cdot \lambda)=\left(d_{i} \sigma\right) \cdot\left(d_{i \cdot \sigma} \lambda\right) .
$$

Since $\zeta(\sigma \cdot \lambda(t))=\zeta(\lambda(t))$, we have

$$
\begin{aligned}
& s_{i}(\sigma \cdot \lambda)(t) \\
= & \left(\lambda^{0 \cdot \sigma}(t), \ldots, \lambda^{i \cdot \sigma}(t), \theta\left(\zeta(\sigma \cdot \lambda(t)), \lambda^{i \cdot \sigma}(t)\right), \ldots, \lambda^{n \cdot \sigma}(t)\right) \\
= & \left(\lambda^{0 \cdot \sigma}(t), \ldots, \lambda^{i \cdot \sigma}(t), \theta\left(\zeta(\lambda(t)), \lambda^{i \cdot \sigma}(t)\right), \ldots, \lambda^{n \cdot \sigma}(t)\right)=\left(s_{i} \sigma\right) \cdot\left(s_{i \cdot \sigma} \lambda\right) .
\end{aligned}
$$

The result follows.

The proof of the following is immediate.

Lemma 3.2.9. Let $\mathbf{p}$ and $\mathbf{q}$ be any two configurations in $F(M, n+1)$. Then there is a commutative diagram

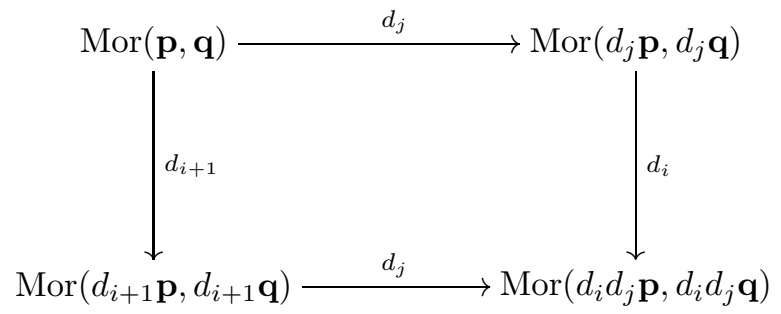

for $0 \leq j \leq i \leq n$.

Use the notation $\wedge_{j}$. in a sequence to indicate the omission of the term indexed by $j$. Let $L_{j, i}(\mathbf{p})$ be the path defined in $F(M, n+1)$ by

$$
L_{j, i}(\mathbf{p})(s)= \begin{cases}\left(p_{0}, \wedge_{j} ., p_{i}, f(\mathbf{p}, s), p_{i+1}, \ldots, p_{n}\right) & j \leq i \\ \mathbf{p} & j=i+1, \\ \left(p_{0}, \ldots, p_{i}, g(\mathbf{p}, s), p_{i+1}, \wedge^{j-1}, p_{n}\right) & j>i+1\end{cases}
$$

where

$$
\begin{gathered}
f(\mathbf{p}, s)=\theta\left((1-s) \zeta(\mathbf{p})+s \zeta\left(d_{j}(\mathbf{p})\right), p_{i}\right) \\
g(\mathbf{p}, s)=\theta\left((1-s) \zeta(\mathbf{p})+s \zeta\left(d_{j-1}(\mathbf{p})\right), p_{i}\right)
\end{gathered}
$$

Observe that $L_{j, i}(\mathbf{p})$ is a path from $L_{j, i}(\mathbf{p})(0)=d_{j} s_{i}(\mathbf{p})$ to

$$
L_{j, i}(\mathbf{p})(1)= \begin{cases}s_{i-1} d_{j}(\mathbf{p}) & j \leq i \\ \mathbf{p} & j=i+1 \\ s_{i} d_{j-1}(\mathbf{p}) & j>i+1\end{cases}
$$

Let $L_{j, i}(\mathbf{p})^{*}, L_{j, i}(\mathbf{q})_{*}$ respectively denote pre- and post-multiplication by this path. 
Lemma 3.2.10. Let $\mathbf{p}$ and $\mathbf{q}$ be two configurations in $F(M, n+1)$. Then the composite $d_{i+1} s_{i}=\mathrm{id}$ and the following diagrams are commutative:

(1)

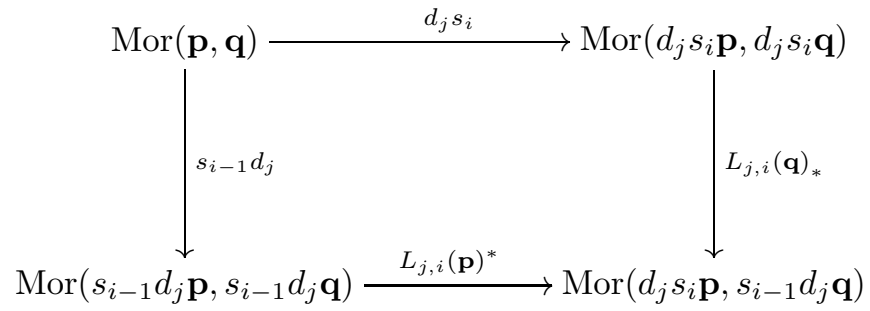

(2)

for $j \leq i$;

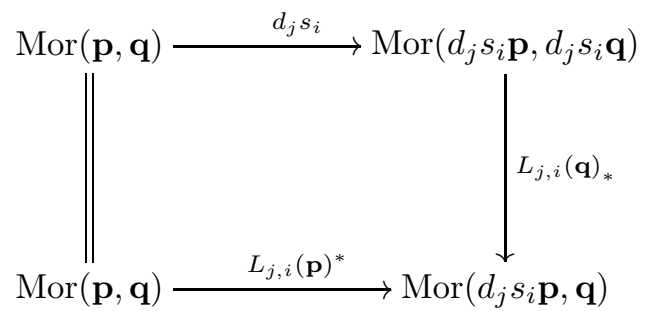

(3)

for $j=i+1$;

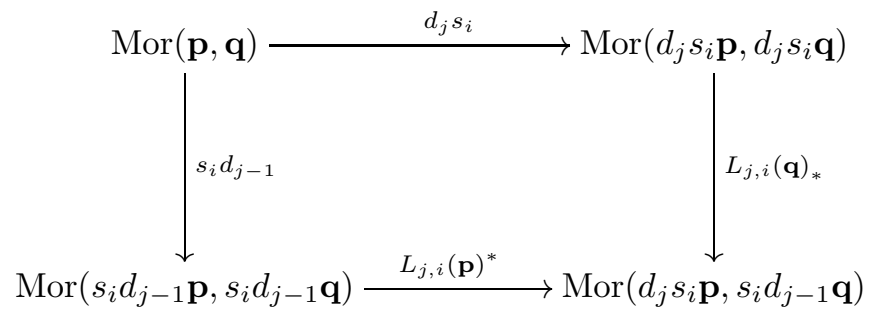

for $j>i+1$.

Proof. Let $\lambda=\left(\lambda^{0}, \ldots, \lambda^{n}\right)$ be a path in $F(M, n+1)$. Then

$d_{j} s_{i}(\lambda)(t)= \begin{cases}\left(\lambda^{0}(t), \wedge^{j} \cdot \lambda^{i}(t), \theta\left(\zeta(\lambda(t)), \lambda^{i}(t)\right), \lambda^{i+1}(t), \ldots, \lambda^{n}(t)\right) & \text { for } j \leq i, \\ \lambda(t) & \text { for } j=i+1, \\ \left(\lambda^{0}(t), \ldots, \lambda^{i}(t), \theta\left(\zeta(\lambda(t)), \lambda^{i}(t)\right), \lambda^{i+1}(t), \wedge^{j-1}, \lambda^{n}(t)\right) & \text { for } j>i+1 .\end{cases}$

On the other hand,

$$
s_{i-1} d_{j}(\lambda)(t)=\left(\lambda^{0}(t), \wedge_{j}, \lambda^{i}(t), \theta\left(\zeta\left(d_{j} \lambda(t)\right), \lambda^{i}(t)\right), \lambda^{i+1}(t), \ldots, \lambda^{n}(t)\right)
$$

for $j<i$ and

$$
s_{i} d_{j-1}(\lambda)(t)=\left(\lambda^{0}(t), \ldots, \lambda^{i}(t), \theta\left(\zeta\left(d_{j-1} \lambda(t)\right), \lambda^{i}(t)\right), \lambda^{i+1}(t), \wedge_{j-1}, \lambda^{n}(t)\right)
$$

for $j>i+1$. This gives the assertion.

Let $\mathbf{p}$ be any configuration in $F(M, n+1)$. For $0 \leq j \leq i \leq n$, we define the path $\tilde{L}_{j, i}(\mathbf{p})$ in $F(M, n+3)$ by

$$
\tilde{L}_{j, i}(\mathbf{p})(s)=\left(p_{0}, \ldots, p_{j}, \tilde{f}(\mathbf{p}, s), \ldots, p_{i}, \tilde{g}(\mathbf{p}, s), \ldots, p_{n}\right),
$$


where $\tilde{f}(\mathbf{p}, s)=\theta\left(s \zeta(\mathbf{p})+(1-s) \zeta\left(s_{i} \mathbf{p}\right), p_{j}\right)$ and $\tilde{g}(\mathbf{p}, s)=\theta\left(s \zeta\left(s_{j} \mathbf{p}\right)+(1-s) \zeta(\mathbf{p}), p_{i}\right)$. Observe that

$$
\tilde{L}_{j, i}(0)=s_{j} s_{i} \mathbf{p} \quad \text { and } \quad \tilde{L}_{j, i}(1)=s_{i+1} s_{j} \mathbf{p} .
$$

Lemma 3.2.11. Let $\mathbf{p}$ and $\mathbf{q}$ be two configurations in $F(M, n+1)$. Then there is a commutative diagram:

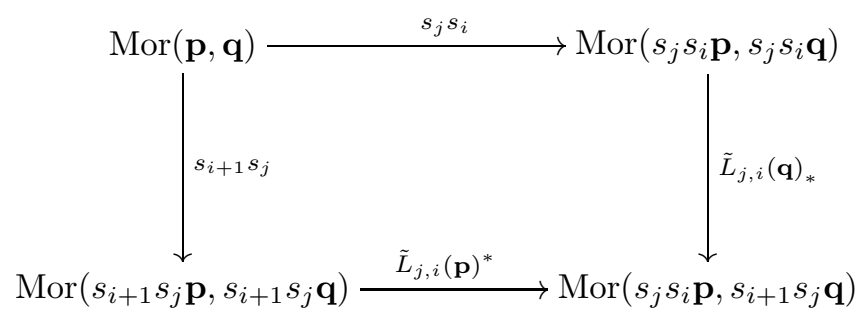

for $0 \leq j \leq i \leq n$.

Proof. Let $\lambda$ be a path from $\mathbf{p}$ to $\mathbf{q}$. Then

$$
\begin{gathered}
s_{j} s_{i}(\lambda)(t)=\left(\lambda^{0}(t), \ldots, \lambda^{j}(t), \theta\left(\zeta\left(s_{i} \lambda(t)\right), \lambda^{j}(t)\right), \ldots, \lambda^{i}(t), \theta\left(\zeta(\lambda(t)), \lambda^{i}(t)\right), \ldots, \lambda^{n}(t)\right), \\
s_{i+1} s_{j}(\lambda)(t)=\left(\lambda^{0}(t), \ldots, \lambda^{j}(t), \theta\left(\zeta(\lambda(t)), \lambda^{j}(t)\right), \ldots, \lambda^{i}(t), \theta\left(\zeta\left(s_{j} \lambda(t)\right), \lambda^{i}(t)\right), \ldots, \lambda^{n}(t)\right) .
\end{gathered}
$$

The result follows.

There is a natural choice of basepoints in $B(M, n+1)$. For the basepoint $w$ of $M$ in Definition 3.2.1, let

$$
w_{i}=\theta(i, w)
$$

for integers $i \geq 0$. Thus by (2) of Definition 3.2.1, for all $i \neq j \geq 0, w_{i} \neq w_{j}$, while by $(1) w_{0}=w$. Let $\mathbf{w}_{n}=\left(w_{0}, \ldots, w_{n}\right)$ be the basepoint for $F(M, n+1)$; then the basepoint of $B(M, n+1)$ is given by the image of $\mathbf{w}_{n}$.

Theorem 3.2.12. Let $M$ be a metric space with a steady flow. Then the sequence of groups $\mathcal{B}(M)^{\pi_{1}}=\left\{\pi_{1}(B(M, n+1))\right\}_{n \geq 0}$ is a crossed simplicial group.

Proof. Let $G_{n}$ be the set of path homotopy classes of paths in $F(M, n+1)$ starting at $\mathbf{w}_{n}$ and ending with a permutation of $\mathbf{w}_{n}$. To each such path $\lambda$, associate the permutation $\mu(\lambda)=\sigma \in S_{n+1}$, where $\lambda(1)=\left(w_{\sigma^{-1}(0)}, \ldots, w_{\sigma^{-1}(n)}\right)$. From our definition of $B(M, n+1)$, we have $\pi_{1}(B(M, n+1))=G_{n}$ as sets. The multiplication in $G_{n}$ induced from $\pi_{1}(B(M, n+1))$ is given as follows. For $i=1,2$, let $\sigma_{i}=$ $\mu\left(\lambda_{i}\right) \in S_{n+1}$, and let $\lambda_{i}$ be a path in $F(M, n+1)$ with $\lambda_{i}(0)=\mathbf{w}_{n}$ and $\lambda_{i}(1)=$ $\left(w_{\sigma_{i}^{-1}(0)}, \ldots, w_{\sigma_{i}^{-1}(n)}\right)$. Then $\left[\lambda_{1}\right]\left[\lambda_{2}\right]$ in $G_{n}$ is represented by the path-product $\lambda_{1} *\left(\sigma_{1} \cdot \lambda_{2}\right)$ from $\left(w_{0}, \ldots, w_{n}\right)$ to $\left(w_{\sigma_{2}^{-1}\left(\sigma_{1}^{-1}(0)\right)}, \ldots, w_{\sigma_{2}^{-1}\left(\sigma_{1}^{-1}(n)\right)}\right)$. Therefore the map $\mu: G_{n} \rightarrow S_{n+1}$ is a group homomorphism.

By assumption (2) in Definition 3.2.1, $\tilde{\theta}=\left.\theta\right|_{\mathbb{R}^{+} \times\{w\}}: \mathbb{R}^{+} \rightarrow M$ is one-to-one and so induces a map

$$
\tilde{\theta}_{n+1}=F(\tilde{\theta}, n+1): F\left(\mathbb{R}^{+}, n+1\right) \rightarrow F(M, n+1)
$$

for each $n$. The subset $\operatorname{Im}\left(\tilde{\theta}: \mathbb{R}^{+} \rightarrow M\right)$ is ordered by $\theta(t, w) \leq \theta\left(t^{\prime}, w\right)$ if $t \leq t^{\prime}$. Thus it follows that $w_{i}<w_{j}$ for $i<j$.

For each $i=0,1, \ldots, n-1$, define a canonical path $\eta_{i}=\left(\eta_{i}^{0}, \ldots, \eta_{i}^{n-1}\right)$ from $(0, \ldots, n-1)$ to $(0, \ldots, i-1, i+1, \ldots, n)$ in $F\left(\mathbb{R}^{+}, n\right)$ by

$$
\eta_{i}^{j}(t)= \begin{cases}j & j \leq i-1, \\ j+t & j \geq i .\end{cases}
$$


Put $\delta_{i}=\tilde{\theta}_{n} \circ \eta_{i}$, a path from $\mathbf{w}_{n-1}$ to $d_{i} \mathbf{w}_{n}=\left(w_{0}, \ldots, w_{i-1}, w_{i+1}, \ldots, w_{n}\right)$ in $F(M, n)$. Define

$$
d_{i}[\lambda]=\left[\delta_{i}\right]\left[d_{i} \lambda\right]\left[\left(d_{i} \sigma\right) \cdot \delta_{\sigma^{-1}(i)}\right]^{-1}
$$

which lies in $G_{n-1}$. This gives a function $d_{i}: G_{n} \rightarrow G_{n-1}$ for $0 \leq i \leq n$. Since $d_{i}[\lambda]$ ends at $\left(w_{\left(d_{i} \sigma\right)^{-1}(0)}, \ldots, w_{\left(d_{i} \sigma\right)^{-1}(n-1)}\right)$, there is a commutative diagram

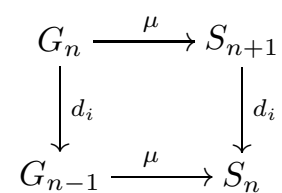

and so the map $\mu: \mathcal{G} \rightarrow \mathcal{S}$ preserves faces.

By assumption (3) in Definition 3.2.1, the point $\theta\left(\zeta\left(\mathbf{w}_{n}\right), w_{i}\right)$ lies in the image of $\tilde{\theta}$ and

$$
w_{i}<\theta\left(\zeta\left(\mathbf{w}_{n}\right), w_{i}\right)<w_{i+1}
$$

for $0 \leq i \leq n$. Thus there is a path $\kappa_{i}$ from $\mathbf{w}_{n+1}$ to

$$
s_{i}\left(w_{0}, \ldots, w_{n}\right)=\left(w_{0}, \ldots, w_{i}, \theta\left(\zeta\left(\mathbf{w}_{n}\right), w_{i}\right), w_{i+1}, \ldots, w_{n}\right)
$$

induced by a path in $F\left(\mathbb{R}^{+}, n+2\right)$ for $0 \leq i \leq n$. Let $[\lambda] \in G_{n}$ with $\mu(\lambda)=\sigma$; in other words, $\lambda(1)=\left(w_{\sigma^{-1}(0)}, \ldots, w_{\sigma^{-1}(n)}\right)=\sigma \cdot \mathbf{w}_{n}$. Then the path $s_{i} \lambda$ runs from $s_{i} \mathbf{w}_{n}$ to

$$
\left(w_{\sigma^{-1}(0)}, \ldots, w_{\sigma^{-1}(i)}, \theta\left(\zeta\left(\sigma \cdot \mathbf{w}_{n}\right), w_{\sigma^{-1}(i)}\right), w_{\sigma^{-1}(i+1)}, \ldots, w_{\sigma^{-1}(n)}\right) .
$$

Since $\zeta\left(\sigma \cdot \mathbf{w}_{n}\right)=\zeta\left(\mathbf{w}_{n}\right)$, the paths $s_{i} \lambda$ and $\left(s_{i} \sigma\right) \cdot \kappa_{\sigma^{-1}(i)}$ have the same final point. Define

$$
s_{i}[\lambda]=\left[\kappa_{i}\right]\left[s_{i} \lambda\right]\left[\left(s_{i} \sigma\right) \cdot \kappa_{\sigma^{-1}(i)}\right]^{-1}
$$

in $G_{n+1}$. Thereby there is the function

$$
s_{i}: G_{n} \rightarrow G_{n+1}
$$

for $0 \leq i \leq n$. Since $s_{i}[\lambda]$ ends at $\left(w_{\left(s_{i} \sigma\right)^{-1}(0)}, \ldots, w_{\left(s_{i} \sigma\right)^{-1}(n+1)}\right)$, there is a commutative diagram

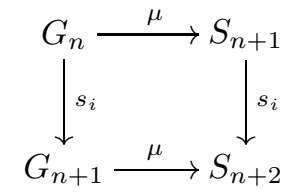

and so the map $\mathcal{G} \rightarrow \mathcal{S}$ preserves degeneracies.

By using Lemmas 3.2.8 3.2 .11 , it is routine to show that $\mathcal{G}$ is a crossed simplicial group. For example, consider the special case that $d_{j} s_{i}=s_{i-1} d_{j}$ for $j<i$. With the convention that $\mathbf{p} \stackrel{\alpha}{-\rightarrow} \mathbf{q}$ denotes a path $\alpha$ running from $\mathbf{p}$ to $\mathbf{q}$, consider the 
diagram

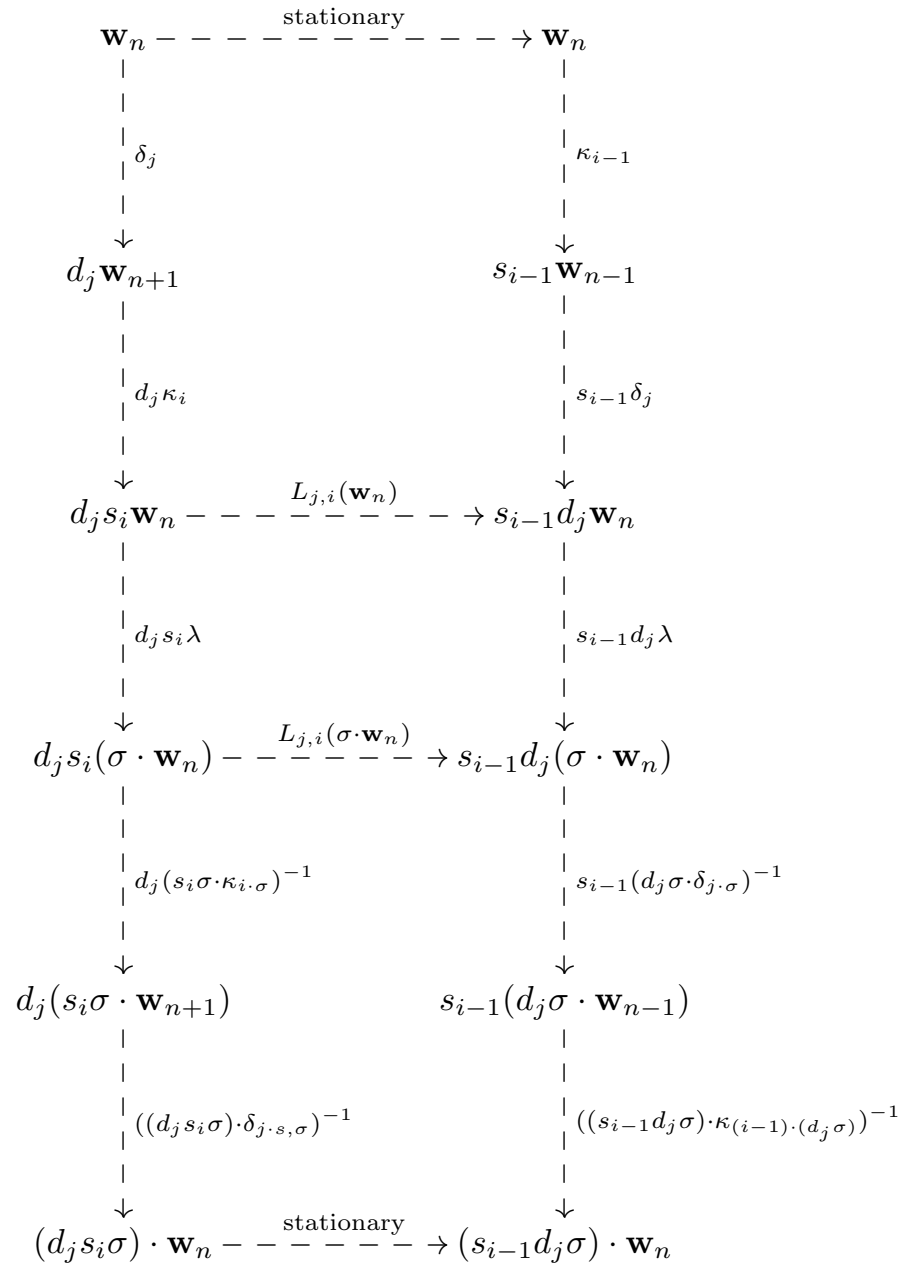

where the left and right columns are the paths that represent $d_{j} s_{i}[\lambda]$ and $s_{i-1} d_{j}[\lambda]$, respectively. By Lemma 3.2.10, the middle square commutes up to path homotopy. Since the paths in the top and bottom squares are induced by paths in $F\left(\mathbb{R}^{+}, n+1\right)$, the top and bottom squares are also commutative diagrams up to path homotopy, as required.

Since $\mathcal{F}(M)^{\pi_{1}}$ is the kernel of the canonical map $\mu: \mathcal{B}(M)^{\pi_{1}} \rightarrow \mathcal{S}$, the following consequence is immediate.

Corollary 3.2.13. Let $M$ be a metric space with a steady flow. Then the sequence of groups $\mathcal{F}(M)^{\pi_{1}}=\left\{\pi_{1}(F(M, n+1))\right\}_{n \geq 0}$ is a simplicial group.

However, in general the sequence of groups $\mathcal{F}(M)^{\pi_{1}}$ need not admit a simplicial group structure, as the next result shows.

Proposition 3.2.14. Let $M=S^{2}$. Then $\mathcal{F}\left(S^{2}\right)^{\pi_{1}}$ does not admit a simplicial group structure. 
Proof. Write $\mathcal{G}$ for $\mathcal{F}\left(S^{2}\right)^{\pi_{1}}$. Thus, $G_{n}=\pi_{1}\left(F\left(S^{2}, n+1\right)\right)$ for $n \geq 0$, with the groups $G_{0}=G_{1}=1$. By Corollary 2.3. $G_{2}=\pi_{1}\left(F\left(S^{2}, 3\right)\right)=\mathbb{Z} / 2$ and

$$
\pi_{1}\left(F\left(S^{2}, 4\right)\right) \cong \pi_{1}\left(S^{2}-Q_{3}\right) \times \pi_{1}\left(F\left(S^{2}, 3\right)\right) \cong F_{2} \times \mathbb{Z} / 2,
$$

where $F_{2}$ denotes the free group of rank 2 . Thus there is a unique element of order 2 in $\pi_{1}\left(F\left(S^{2}, 4\right)\right)$. Let $\sigma$ be the generator for $\pi_{1}\left(F\left(S^{2}, 3\right)\right)$. Now suppose that $\mathcal{G}=\mathcal{F}\left(S^{2}\right)^{\pi_{1}}$ were a simplicial group. Then $\left(s_{i} \sigma\right)^{2}=s_{i}\left(\sigma^{2}\right)=1$ for $0 \leq i \leq 2$ and so $s_{0} \sigma=s_{1} \sigma=s_{2} \sigma$. On the other hand, by the simplicial identities,

$$
\sigma=d_{0} s_{0} \sigma=d_{0} s_{1} \sigma=s_{0} d_{0} \sigma=s_{0}(1)=1,
$$

a contradiction.

Note. It can happen that the sequence of groups $\mathcal{F}(M)^{\pi_{1}}$ is a simplicial group although $M$ does not have a steady flow. For example, if $M$ is a simply connected manifold with $\operatorname{dim} M \geq 3$, then $\pi_{1}(F(M, n+1))=1$ for each $n$. In this case, nontrivial information for higher-dimensional manifolds is given in Section 5, for the sequence of sets $\{[A, F(M, n+1)]\}_{n \geq 0}$ for general spaces $A$.

\section{4. $\Delta$-GROUPS ON CONFIGURATIONS}

4.1. $\Delta$-groups. A sequence of sets $\mathcal{X}=\left\{X_{n}\right\}_{n \geq 0}$ is called a $\Delta$-set if there are functions $d_{i}: X_{n} \rightarrow X_{n-1}$ for $0 \leq i \leq n$ such that $d_{j} d_{i}=d_{i} d_{j+1}$ for $i \leq j$. $f=\left\{f_{n}\right\}_{n \geq 0}: \mathcal{X} \rightarrow \mathcal{X}^{\prime}$ is called a $\Delta$-map if $d_{i} f_{n}=f_{n-1} d_{i}$. The set of $\Delta$-maps from $\mathcal{X}$ to $\mathcal{X}^{\prime}$ is denoted by $\operatorname{Map}\left(\mathcal{X}, \mathcal{X}^{\prime}\right)_{0}$. A $\Delta$-group $\mathcal{G}=\left\{G_{n}\right\}_{n \geq 0}$ consists of a $\Delta$-set $\mathcal{G}$ for which each $G_{n}$ is a group and each $d_{i}$ is a group homomorphism. The Moore complex $N \mathcal{G}=\left\{N_{n} \mathcal{G}\right\}_{n \geq 0}$ of a $\Delta$-group $\mathcal{G}$ is defined by

$$
N_{n} \mathcal{G}=\bigcap_{i=1}^{n} \operatorname{Ker}\left(d_{i}: G_{n} \rightarrow G_{n-1}\right) .
$$

Lemma 4.1.1. Let $\mathcal{G}$ be a $\Delta$-group. Then $d_{0}\left(N_{n} \mathcal{G}\right) \subseteq N_{n-1} \mathcal{G}$ and $N \mathcal{G}$ with $d_{0}$ is a chain complex of groups.

Proof. The assertion follows from the relation

$$
d_{j} d_{0}=d_{0} d_{j+1}
$$

for $j \geq 0$.

Let $\mathcal{G}$ be a $\Delta$-group. An element in $\mathrm{B}_{n} \mathcal{G}=d_{0}\left(N_{n+1} \mathcal{G}\right)$ is called a Moore boundary and an element in $\mathrm{Z}_{n} \mathcal{G}=\operatorname{Ker}\left(d_{0}: N_{n} \mathcal{G} \rightarrow N_{n-1} \mathcal{G}\right)$ is called a Moore cycle. The $n$-th homotopy $\pi_{n}(\mathcal{G})$ is defined to be the coset

$$
\pi_{n}(\mathcal{G})=H_{n}(N \mathcal{G})=\mathrm{Z}_{n} \mathcal{G} / \mathrm{B}_{n} \mathcal{G} .
$$

The homotopy set $\pi_{n}(\mathcal{G})$ need not be a group in general because the boundaries $\mathrm{B}_{n} \mathcal{G}$ need not form a normal subgroup of the cycles $\mathrm{Z}_{n} \mathcal{G}$ in general. For instance, let $B$ be a nonnormal subgroup of $A$ and let $\mathcal{G}$ be a $\Delta$-group given by $G_{0}=A$, $G_{1}=B$ and $G_{n}=\{1\}$ for $n \geq 2$ with the inclusion $d_{0}: G_{1} \rightarrow G_{0}$ and the trivial map $d_{1}: G_{1} \rightarrow G_{0}$. Then $\pi_{0}(\mathcal{G})$ is the coset $B / A$. Under a weak condition, $\pi_{n}(\mathcal{G})$ has a group structure.

Lemma 4.1.2. Let $\mathcal{G}$ be a $\Delta$-group. Then, for each $n \geq 0, \mathrm{~B}_{n} \mathcal{G}$ is a normal subgroup of $\operatorname{Im}\left(d_{0}: G_{n+1} \rightarrow G_{n}\right)$. In particular, $\pi_{n}(\mathcal{G})$ is a group if $Z_{n}(\mathcal{G})$ is contained in $\operatorname{Im}\left(d_{0}: G_{n+1} \rightarrow G_{n}\right)$. 
Proof. Let $x \in \mathrm{B}_{n} \mathcal{G}$ and let $w$ be such that there exists $\tilde{w} \in G_{n+1}$ with $d_{0} \tilde{w}=w$. By definition, there exists $y \in N_{n+1} \mathcal{G}$ such that $d_{0} y=x$. Now

$$
d_{j}[y, \tilde{w}]=\left[1, d_{j} \tilde{w}\right]=1
$$

for $j \geq 1$ and $d_{0}[y, \tilde{w}]=[x, w]$. Thus the commutator $[x, w] \in \mathrm{B}_{n} \mathcal{G}$ as required.

In the above, we have defined $\pi_{n}(\mathcal{G})$ with reference to $d_{0}$. In principle, using a different $d_{i}$ could result in different homotopy sets (as in the example above), although always the cycles are given by

$$
\mathrm{Z}_{n} \mathcal{G}=\bigcap_{0 \leq j \leq n} \operatorname{Ker}\left(d_{j}: G_{n} \rightarrow G_{n-1}\right) .
$$

By the relations $d_{k} d_{i}=d_{i-1} d_{k}$ for $k<i$ and $d_{k} d_{i}=d_{i} d_{k+1}$ for $k \geq i$, we also have

$$
d_{i}\left(\bigcap_{j \neq i} \operatorname{Ker}\left(d_{j}: G_{n+1} \rightarrow G_{n}\right)\right) \subseteq \mathrm{Z}_{n} \mathcal{G}
$$

for each $0 \leq i \leq n+1$. So in general the boundary set is well-defined; however, it may differ from $\mathrm{B}_{n} \mathcal{G}$. We now investigate circumstances in which these possible sets of boundaries coincide.

Let $\mathcal{X}$ be a $\Delta$-set. The elements $x_{0}, \ldots, x_{i-1}, x_{i+1}, \ldots, x_{n} \in X_{n-1}$ are called matching faces with respect to $i$ if

$$
d_{j} x_{k}=d_{k} x_{j+1}
$$

for $k \leq j$ and $k, j+1 \neq i$. Recall that a $\Delta$-set $\mathcal{X}$ is fibrant if it satisfies the following homotopy extension condition for each $i$ :

Let $x_{0}, \ldots, x_{i-1}, x_{i+1}, \ldots, x_{n} \in X_{n-1}$ be any elements that are matching faces with respect to $i$. Then there exists an element $w \in X_{n}$ such that $d_{j} w=x_{j}$ for $j \neq i$.

The nondegenerate $n$-simplex $\Delta[n]$ is defined as:

$$
\Delta[n]_{k}=\left\{\left(i_{0}, \ldots, i_{k}\right) \in \mathbb{Z}^{k+1} \mid 0 \leq i_{0}<i_{1}<\cdots<i_{k} \leq n\right\}
$$

with the usual face maps given by deleting coordinates. (Note. The difference between the nondegenerate $n$-simplex and the usual simplicial $n$-simplex is that a simplicial $n$-simplex allows degeneracies, and so the strict inequality notation $<$ in the above definition is replaced by $\leq$ in the definition of simplicial $n$-simplex.) Let $\mathcal{X}$ be a $\Delta$-set and let $x \in X_{n}$. Then there is a unique map of $\Delta$-sets

$$
f_{x}: \Delta[n] \rightarrow X_{n}
$$

such that $f_{x}\left(\sigma_{n}\right)=x$, where $\sigma_{n}=(0, \ldots, n)$. The map $f_{x}$ is called the representing map of the element $x$. Let $\Lambda^{i}[n]$ be the $\Delta$-subset of $\Delta[n]$ generated by $d_{j} \sigma_{n}$ for $j \neq i$. In geometry, $\Lambda^{i}[n]$ is obtained from the simplex $\Delta[n]$ by deleting the $i$-face. The elements $x_{0}, \ldots, x_{i-1}, x_{i+1}, \ldots, x_{n} \in X_{n-1}$ are matching faces with respect to $i$ if and only if there is a map of $\Delta$-sets $\phi: \Lambda^{i}[n] \rightarrow \mathcal{X}$ such that $\phi\left(d_{j} \sigma_{n}\right)=f_{x_{j}}$ for each $j \neq i$. It follows that any $\Delta$-map sends matching faces to matching faces. Thus $\mathcal{X}$ is fibrant if and only if the induced mapping in dimension zero

$$
\operatorname{Map}(\Delta[n], \mathcal{X})_{0} \rightarrow \operatorname{Map}\left(\Lambda^{i}[n], \mathcal{X}\right)_{0}
$$

is onto whenever $0 \leq i \leq n$. 
Proposition 4.1.3. Let $\mathcal{G}$ be a fibrant $\Delta$-group. Then

(1) $\mathrm{B}_{n} \mathcal{G}$ is a normal subgroup of $\mathrm{Z}_{n} \mathcal{G}$ for each $n$;

(2) $d_{i}\left(\bigcap_{j \neq i} \operatorname{Ker}\left(d_{j}: G_{n+1} \rightarrow G_{n}\right)\right)=\mathrm{B}_{n} \mathcal{G}$ for each $0 \leq i \leq n+1$;

(3) $\pi_{n}(\mathcal{G})$ is an abelian group for $n \geq 1$.

Proof. (1). Let $x_{0} \in \mathrm{Z}_{n} \mathcal{G}$ and let $x_{1}=x_{2}=\cdots=x_{n}=1$. Then the elements $x_{i}$ are matching faces with respect to $n+1$, and so there is an element $y \in G_{n+1}$ such that $d_{0} y=x_{0}$ and $d_{j} y=1$ for $0<j<n+1$. So the result follows from the preceding lemma.

(2). Fix $i>0$. Let $x \in G_{n+1}$ such that $d_{j} x=1$ for $j \neq i$. Let $x_{0}=x$ and $x_{k}=1$ for $k \neq 0, i+1$. Then $x_{0}, \ldots, x_{i}, x_{i+2}, \ldots, x_{n+2}$ are matching faces with respect to $i+1$, and so there is an element $z \in G_{n+2}$ such that $d_{0} z=x_{0}=x$ and $d_{k} z=1$ for $k \neq 0, i+1$. Let $y=d_{i+1} z$. Then

$$
d_{i} x=d_{i} d_{0} z=d_{0} d_{i+1} z=d_{0} y,
$$

where

$$
d_{j} y=d_{j} d_{i+1} z=d_{i+1} d_{j+1} z=1
$$

for $j \geq i+1$, and

$$
d_{j} y=d_{j} d_{i+1} z=d_{i} d_{j} z=1
$$

for $0<j \leq i$, so that $y \in N_{n+1} \mathcal{G}$. Thus

$$
d_{i}\left(\bigcap_{j \neq i} \operatorname{Ker}\left(d_{j}: G_{n+1} \rightarrow G_{n}\right)\right) \subseteq \mathrm{B}_{n} \mathcal{G} .
$$

Similarly, notice that

$$
\mathrm{B}_{n} \mathcal{G}=d_{0}\left(\bigcap_{j \neq 0} \operatorname{Ker}\left(d_{j}: G_{n+1} \rightarrow G_{n}\right)\right) \subseteq d_{i}\left(\bigcap_{j \neq i} \operatorname{Ker}\left(d_{j}: G_{n+1} \rightarrow G_{n}\right)\right) .
$$

This yields Assertion (2).

(3). Let $z_{0}, z_{1} \in \mathrm{Z}_{n} \mathcal{G}$ with $n \geq 1$. Then there exists an element $w_{0} \in G_{n+1}$ such that $d_{0} w_{0}=z_{0}$ and $d_{k} w_{0}=1$ for $k>1$, because the elements

$$
x_{0}=z_{0}, \quad x_{2}=1, \ldots, x_{n+1}=1
$$

are matching faces with respect to 1 . Similarly, there is an element $w_{1} \in G_{n+1}$ such that $d_{0} w_{1}=z_{1}, d_{1} w_{1}=1$ and $d_{j} w_{1}=1$ for $j>2$. It follows that $d_{j}\left[w_{0}, w_{1}\right]=1$ for $j>0$ and $d_{0}\left[w_{0}, w_{1}\right]=\left[z_{0}, z_{1}\right]$. Hence $\left[\mathrm{Z}_{n} \mathcal{G}, \mathrm{Z}_{n} \mathcal{G}\right] \subseteq \mathrm{B}_{n} \mathcal{G}$.

Proposition 4.1.4. The functor $N: \mathcal{G} \rightarrow N \mathcal{G}$ is a left exact functor from $\Delta$ groups to chain complexes of groups. Moreover, $N$ is an exact functor from fibrant $\Delta$-groups to chain complexes of groups, and so $\left\{\pi_{n}\right\}$ is a derived functor from fibrant $\Delta$-groups to sequences of abelian groups.

Proof. Clearly $N$ is left exact. Let

$$
1 \longrightarrow \mathcal{G}^{\prime} \longrightarrow \mathcal{G} \stackrel{\epsilon}{\longrightarrow} \mathcal{G}^{\prime \prime} \longrightarrow 1
$$

be a short exact sequence of $\Delta$-groups. It suffices to show that when $\mathcal{G}^{\prime}$ is fibrant, $N \mathcal{G} \rightarrow N \mathcal{G}^{\prime \prime}$ is onto. Let $x \in N_{n} \mathcal{G}^{\prime \prime}$ and let $y \in G_{n}$ such that $\epsilon(y)=x$. Then $d_{j} y \in G_{n-1}^{\prime}$ for $j \geq 1$. Since $\left\{d_{j} y\right\}_{j \geq 1}$ are matching faces with respect to 0 , there exists an element $z \in G_{n}^{\prime}$ such that $d_{j} z=d_{j} y$ for $j \geq 1$. Thus $y z^{-1} \in N_{n} \mathcal{G}$ with $\epsilon\left(y z^{-1}\right)=x$ and hence the result. 
For any $\Delta$-group $\mathcal{G}$, the Moore path $P \mathcal{G}$ of $\mathcal{G}$ is defined by

$$
(P \mathcal{G})_{n}=\left\{x \in G_{n+1} \mid d_{1} \circ d_{2} \circ \cdots \circ d_{n+1}(x)=1\right\}
$$

with $d_{j}^{P}:(P \mathcal{G})_{n} \rightarrow(P \mathcal{G})_{n-1}$, where $d_{j}^{P}(x)=d_{j+1}(x)$ for $0 \leq j \leq n$. The map $p: P \mathcal{G} \rightarrow \mathcal{G}$ is defined by

$$
p:(P \mathcal{G})_{n} \hookrightarrow G_{n+1} \stackrel{d_{0}}{\longrightarrow} G_{n} .
$$

Since $d_{j} d_{0}=d_{0} d_{j+1}$, the map $p$ is a morphism of $\Delta$-groups. The Moore loop $\Omega \mathcal{G}$ of $\mathcal{G}$ is defined to be the kernel of $p$. In other words,

$$
(\Omega \mathcal{G})_{n}=\left\{x \in G_{n+1} \mid d_{0}(x)=1 \text { and } d_{1} \circ d_{2} \circ \cdots \circ d_{n+1}(x)=1\right\}
$$

and each face $d_{j}^{\Omega}=d_{j+1}$.

Proposition 4.1.5. Let $\mathcal{G}$ be a $\Delta$-group. Then

(1) For each $n \geq 0, \pi_{n}(\Omega \mathcal{G})=\pi_{n+1}(\mathcal{G})$ if and only if

$$
d_{1}\left(\bigcap_{j \neq 1} \operatorname{Ker}\left(d_{j}: G_{n+1} \rightarrow G_{n}\right)\right)=\mathrm{B}_{n} \mathcal{G} .
$$

(2) If $\mathcal{G}$ is fibrant, then $P \mathcal{G}$ and $\Omega \mathcal{G}$ are fibrant. In particular, for any fibrant $\Delta$-group $\mathcal{G}$ it is always true that $\pi_{*}(\Omega \mathcal{G})=\pi_{*+1}(\mathcal{G})$ and $\pi_{*}(P \mathcal{G})=1$.

Proof. Statement (1) is a routine calculation. To check statement (2), let

$$
x_{0}, \ldots, x_{i-1}, x_{i+1}, \ldots, x_{n} \in(P \mathcal{G})_{n-1}
$$

be matching faces with respect to $i$. Since $p$ is a $\Delta$-map,

$$
d_{0} x_{0}, \ldots, d_{0} x_{i-1}, d_{0} x_{i+1}, \ldots, d_{0} x_{n} \in G_{n-1}
$$

are also matching faces with respect to $i$. By the fibrant condition for $\mathcal{G}$, there exists $v \in G_{n}$ such that $d_{j} v=d_{0} x_{j}$ for all $j \neq i$. Observe that

$$
v, x_{0}, \ldots, x_{i-1}, x_{i+1}, \ldots, x_{n} \in G_{n}
$$

are matching faces with respect to $i+1$. The result follows from another application of the fibrant condition. The case of $\Omega \mathcal{G}$ is straightforward. The last assertion follows from the two previous propositions.

Let $R_{n} \mathcal{G}=\left\{\left(R_{n} \mathcal{G}\right)_{q}\right\}_{q \geq 0}$ be the sequence of groups defined by $\left(R_{n} \mathcal{G}\right)_{q}=1$ for $q \leq n$ and, for $q>n,\left(R_{n} \mathcal{G}\right)_{q}$ consists of all elements $x \in G_{q}$ such that

$$
d_{i_{1}} \circ \cdots \circ d_{i_{q-n}}(x)=1
$$

for all sequences $0 \leq i_{1}<\cdots<i_{q-n} \leq q$. Then $R_{n} \mathcal{G}$, with each face $d_{i}^{R}=d_{i}$, is a $\Delta$-subgroup of $\mathcal{G}$ such that each $\left(R_{n} \mathcal{G}\right)_{q}$ is a normal subgroup of $G_{q}$. Let $\mathrm{P}_{n} \mathcal{G}=\mathcal{G} / R_{n} \mathcal{G}$ be the $\Delta$-quotient group of $\mathcal{G}$. The tower of $\Delta$-groups

$$
\mathcal{G} \rightarrow \cdots \rightarrow \mathrm{P}_{n} \mathcal{G} \rightarrow \mathrm{P}_{n-1} \mathcal{G} \rightarrow \cdots \rightarrow \mathrm{P}_{0} \mathcal{G}
$$

is called the Moore-Postnikov system of $\mathcal{G}$. Let $K_{n} \mathcal{G}$ be the kernel of $\mathrm{P}_{n} \mathcal{G} \longrightarrow$ $\mathrm{P}_{n-1} \mathcal{G}$. 
Proposition 4.1.6. Let $\mathcal{G}$ be a $\Delta$-group. Then

(1) The Moore complex of $\mathrm{P}_{n} \mathcal{G}$ is given by

$$
N_{q} \mathrm{P}_{n} \mathcal{G}= \begin{cases}1 & q \geq n+2, \\ N_{n+1} \mathcal{G} / \mathrm{Z}_{n+1} \mathcal{G} & q=n+1, \\ N_{q} \mathcal{G} & q \leq n\end{cases}
$$

(2) $\pi_{j}\left(\mathrm{P}_{n} \mathcal{G}\right)=1$ for $j>n$, and $\pi_{j}(\mathcal{G}) \longrightarrow \pi_{j}\left(\mathrm{P}_{n} \mathcal{G}\right)$ is an isomorphism for $j \leq n$.

(3) $\pi_{j}\left(K_{n} \mathcal{G}\right)=1$ for $j \neq n$, and $\pi_{n}\left(K_{n} \mathcal{G}\right) \cong \pi_{n}(\mathcal{G})$.

Proof. Assertions (2) and (3) follow immediately from assertion (1), using Proposition 4.1.4

(1). Let $\theta: \mathcal{G} \rightarrow \mathrm{P}_{n} \mathcal{G}$ be the quotient map. For any $x \in N_{q} \mathrm{P}_{n} \mathcal{G}$, there exists an element $y \in G_{q}$ such that $\theta(y)=x$. If $q \leq n+1$, then $y \in N_{q} \mathcal{G}$ because $G_{s}=\left(\mathrm{P}_{n} \mathcal{G}\right)_{s}$ for $s \leq n$. Thus

$$
N \theta: N_{q} \mathcal{G} \rightarrow N_{q} \mathrm{P}_{n} \mathcal{G}
$$

is onto for $q \leq n+1$. Clearly, $N \theta: N_{q} \mathcal{G} \rightarrow N_{q} \mathrm{P}_{n} \mathcal{G}$ is an isomorphism for $q \leq n$. Since $N_{n+1} R_{n} \mathcal{G}=\left(R_{n} \mathcal{G}\right)_{n+1}=\mathrm{Z}_{n+1} \mathcal{G}$, it follows that $N_{n+1} \mathrm{P}_{n} \mathcal{G}=N_{n+1} \mathcal{G} / \mathrm{Z}_{n+1} \mathcal{G}$. Now assume that $q>n+1$. From the identity $d_{s} d_{0}=d_{0} d_{s+1}$ for all $s$, the iterated faces

$$
d_{i_{1}} \circ \cdots \circ d_{i_{q-n}}(y)=1
$$

for any sequence $0 \leq i_{1}<\cdots<i_{q-n} \leq q$, and so $y \in\left(R_{n} \mathcal{G}\right)_{q}$ or $N_{q} \mathrm{P}_{n} \mathcal{G}=1$ for $q>n+1$. This finishes the proof.

4.2. $\Delta$-groups induced by configurations. A space $M$ is said to have a good basepoint $w_{0}$ if there is a continuous injection $\tilde{\theta}: \mathbb{R}^{+} \rightarrow M$ with $\tilde{\theta}(0)=w_{0}$. (This is clearly equivalent to the existence of an embedding of $[0,1]$ in $M$ that sends 0 to $w_{0}$, also known as a whisker.) Thus in particular if a space $M$ contains a subspace $A$ such that

(1) $A$ is a metric space, and

(2) $A$ has a steady flow,

then $M$ has a good basepoint. A cell complex of positive dimension also has a good basepoint.

Recall that the face $d_{i}: F(M, n+1) \rightarrow F(M, n)$ is given by

$$
d_{i}\left(x_{0}, \ldots, x_{n}\right)=\left(x_{0}, \ldots, x_{i-1}, x_{i+1}, \ldots, x_{n}\right)
$$

for $0 \leq i \leq n$. The formula $d_{j} d_{i}=d_{i} d_{j+1}$ for $i \leq j$ is evident. The basepoint for $F(M, n+1)$ is $\left(w_{0}, \ldots, w_{n}\right)$, where $w_{0}$ is a good basepoint for $M$ and $w_{i}=\tilde{\theta}(i)$.

A crossed $\Delta$-group is a $\Delta$-set $\mathcal{G}=\left\{G_{n}\right\}_{n \geq 0}$ for which each $G_{n}$ is a group, together with a group homomorphism $\mu: G_{n} \stackrel{\rightarrow}{\rightarrow} S_{n+1}, g \mapsto \mu_{g}$ for each $n$, such that

(i) the collection of homomorphisms $\mu$ forms a $\Delta$-map, and

(ii) for $0 \leq i \leq n, d_{i}\left(g g^{\prime}\right)=d_{i}(g) d_{i \cdot \mu_{g}}\left(g^{\prime}\right)$.

Proposition 4.2.1. Let $M$ be a space with a good basepoint. Then

(1) $\mathcal{B}(M)^{\pi_{1}}$ is a crossed $\Delta$-group,

(2) $\mathcal{F}(M)^{\pi_{1}}$ is a $\Delta$-group,

(3) $\pi_{n}\left(\Omega \mathcal{F}(M)^{\pi_{1}}\right)=\pi_{n+1}\left(\mathcal{F}(M)^{\pi_{1}}\right)$ for each $n \geq 0$. 
Proof. Parts (1) and (2) follow from the proof of Theorem 3.2.12, and Corollary 3.2.13 Part (3) follows from part (1) of Proposition 4.1.5 by considering the transposition $\tau$ sending $\left(x_{0}, x_{1}, x_{2}, \ldots, x_{n+1}\right)$ to $\left(x_{1}, x_{0}, x_{2}, \ldots, x_{n+1}\right)$. Write

$$
K=\bigcap_{j \neq 1} \operatorname{Ker}\left(d_{j}: \pi_{1}(F(M, n+2)) \rightarrow \pi_{1}(F(M, n+1))\right) .
$$

It is easy to check that $\tau$ induces a bijection $\tau: K \rightarrow N_{n+1} \mathcal{F}(M)^{\pi_{1}}$ and commuting diagram

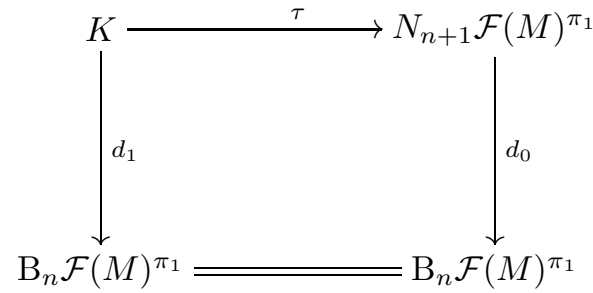

This gives the result.

Recall that an element in $\pi_{1}(B(M, n))$ is called a braid of $n$ strings over $M$. Observe that any braid over $M$ can be described as a path homotopy class in $F(M, n)$. A braid is called Brunnian if it becomes a trivial braid when any one of its strings is removed. In the terminology of $\Delta$-groups, a braid

$$
\beta \in \pi_{1}(B(M, n+1))=\mathcal{B}(M)_{n}^{\pi_{1}}
$$

is Brunnian if and only if $d_{i} \beta=1$ for $0 \leq i \leq n$. In other words, the Brunnian braids over $M$ are the Moore cycles in the $\Delta$-group $\mathcal{B}(M)^{\pi_{1}}$. The group of Brunnian braids of $n$ strings over $M$ is denoted by $\operatorname{Brun}_{n}(M)$. A pure braid of $n$ strings over $M$ means an element in $\pi_{1}(F(M, n))$. Clearly a braid $\beta$ is pure if and only if $\beta$ lies in the kernel of the homomorphism $\mu: \pi_{1}(B(M, n)) \rightarrow S_{n}$. (See section 3.1 for the $\operatorname{map} \mu$.)

Proposition 4.2.2. Let $\beta$ be a Brunnian braid of $n$ strings over a space $M$ with a good basepoint. If $n \geq 3$, then $\beta$ is a pure braid.

Proof. Let $\beta$ be a Brunnian braid of $n$ strings over $M$. Then $\beta$ is a Moore cycle in $\mathcal{B}(M)_{n-1}^{\pi_{1}}$ and so $\mu(\beta)$ is a Moore cycle in $\mathcal{S}=\left\{S_{n+1}\right\}_{n \geq 0}$. By direct calculation, it follows that $\mathrm{Z}_{1} \mathcal{S}=S_{2}$ and $\mathrm{Z}_{n-1} \mathcal{S}=\{1\}$ for $n \geq 3$, giving the result.

In the case where $M$ is a manifold without boundary, the map

$$
d_{i}: F(M, n+1) \rightarrow F(M, n)
$$

is a fibre bundle projection with fibre $M-Q_{n}$, where $Q_{n}=\left\{q_{0}, \ldots, q_{n-1}\right\}$; see [26]. The following will be used in Section 6 .

Definition 4.2.3. A sequence of spaces $\mathcal{E}=\left\{E_{n}\right\}_{n \geq 0}$ is called a $\Delta$-bundle if

(1) there exist faces $d_{i}: E_{n} \rightarrow E_{n-1}$ such that $d_{i}$ is a fibre bundle projection, with the same fibre for $0 \leq i \leq n$;

(2) $d_{j} d_{i}=d_{i} d_{j+1}: E_{n} \rightarrow E_{n-2}$ for $0 \leq i \leq j<n$. 
A $\Delta$-bundle $\mathcal{E}$ is called crossed if each $E_{n}$ admits a left $S_{n+1}$-action such that the following diagram commutes:

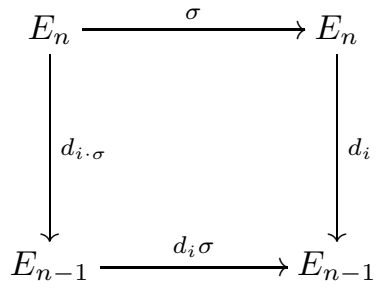

for $\sigma \in S_{n+1}$ and $0 \leq i \leq n$.

Let $\mathcal{E}=\left\{E_{n}\right\}_{n \geq 0}$ be a $\Delta$-bundle such that each $E_{n}$ is path-connected. Define $\tilde{\Omega} \mathcal{E}$ to be the sequence of spaces having $(\tilde{\Omega} \mathcal{E})_{n}$ as the fibre of $d_{0}: E_{n+1} \rightarrow E_{n}$, with faces $d_{j}^{\tilde{\Omega}}=\left.d_{j+1}\right|_{\tilde{\Omega} \mathcal{E}}$. Then $\tilde{\Omega} \mathcal{E}$ is a $\Delta$-space, meaning that each face is continuous.

Proposition 4.2.4. Let $\mathcal{E}$ be a $\Delta$-bundle such that each $E_{n}$ is path-connected. Suppose that $E_{0}$ is simply-connected. Then there is an exact sequence of $\Delta$-groups

$$
\mathcal{E}^{\pi_{2}} \longrightarrow(\tilde{\Omega} \mathcal{E})^{\pi_{1}} \longrightarrow \Omega\left(\mathcal{E}^{\pi_{1}}\right) \longrightarrow 1 \text {. }
$$

Proof. From the commutative diagram of fibrations

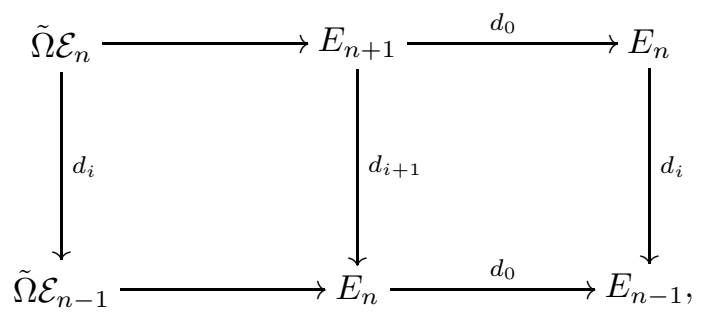

there is a commutative diagram of exact sequences

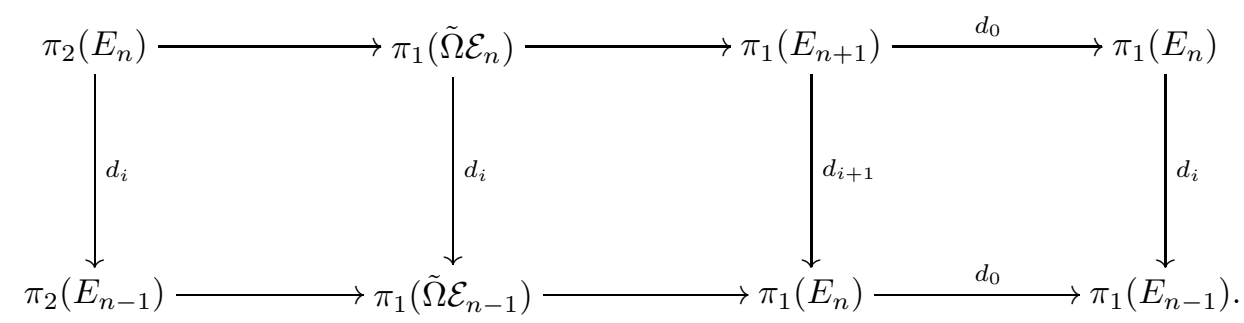

Since $\pi_{1}\left(E_{0}\right)=1$, it follows that

$$
\Omega\left(\mathcal{E}^{\pi_{1}}\right)_{n}=\operatorname{Ker}\left(d_{0}: \pi_{1}\left(E_{n+1}\right) \rightarrow \pi_{1}\left(E_{n}\right)\right)
$$

with $d_{i}^{\Omega \mathcal{E}^{\pi_{1}}}=d_{i+1}^{\mathcal{E}_{1}}$, and hence the result.

Proposition 4.2.5. Let $M$ be a surface. Then $\operatorname{Brun}_{n}(M)$ is a free group for $n \geq 4$ in general, and for $n \geq 3$ when $M$ is not $S^{2}$. (However $\operatorname{Brun}_{3}\left(S^{2}\right)=\mathbb{Z} / 2$.)

Proof. From the fibre sequence

$$
M-Q_{n-1} \longrightarrow F(M, n) \stackrel{d_{0}}{\longrightarrow} F(M, n-1),
$$


there is an exact sequence

$$
\pi_{2}(F(M, n-1)) \stackrel{\partial_{*}}{\longrightarrow} \pi_{1}\left(M-Q_{n-1}\right) \longrightarrow \pi_{1}(F(M, n)) \stackrel{d_{0 *}}{\longrightarrow} \pi_{1}(F(M, n-1)) .
$$

It is standard (see [26]) that $F(M, n)$ is a $K(\pi, 1)$ in case $M$ is any surface not

equal to $S^{2}$ or $\mathbb{R P}^{2}$. For $M=\mathbb{R P}^{2}$ and $n \geq 3$, since $\mathbb{R P}^{2}-Q_{n-1} \simeq \bigvee_{j=1}^{n-1} S^{1}$, from the above exact sequence via the fact that $\partial_{*}$ maps into the center of the free group $F_{n-1}$ and $F_{n-1}$ is centerless for $n \geq 3, \operatorname{Brun}_{n}\left(\mathbb{R P}^{2}\right)$ is a subgroup of the free group

$$
\pi_{1}\left(\bigvee_{j=1}^{n-1} S^{1}\right)=F_{n-1}
$$

and thus it is free. In case $M=S^{2}$ and $n \geq 4$, Corollary 2.3 gives a homotopy equivalence

$$
F(M, n) \simeq F\left(\mathbb{R}^{2}-Q_{2}, n-3\right) \times \mathbb{R P}^{3} .
$$

By the previous case, $\pi_{2}\left(F\left(\mathbb{R}^{2}-Q_{2}, n-3\right)\right)=0$, as is $\pi_{2}\left(\mathbb{R P}^{3}\right)$. So always

$$
\pi_{2}(F(M, n-1))=0
$$

for the asserted range of $n$. Therefore

$$
\operatorname{Ker}\left(d_{0 *}: \pi_{1}(F(M, n)) \rightarrow \pi_{1}(F(M, n-1))\right)
$$

is isomorphic to the free group $\pi_{1}\left(M-Q_{n-1}\right)$. Thus $\operatorname{Brun}_{n}(M)$ is a subgroup of the free group $\pi_{1}\left(M-Q_{n-1}\right)$, hence the result. In the special case of $S^{2}$ with $n=3$, notice that $\pi_{1}\left(F\left(S^{2}, 3\right)\right)=\mathbb{Z} / 2$ by Corollary 2.3 , while $\pi_{1}\left(F\left(S^{2}, 2\right)\right)=1$.

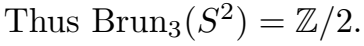

Remark 4.2.6. There was a problem of Makanin in Kourovka Notebook (Problem Book in group theory) in 1980 that Brunnian braids over the disk (called smooth braids in this book) form a free subgroup, with generators to be described. Gurzo gave a solution in the 1981 Leningrad Algebra conference. A published solution was given by Johnson 39.

\section{Proof of Theorem 1.4}

Let $M$ be a space with a good basepoint $w_{0}$ as described in Subsection 4.2. Thus the basepoint for $F(M, n+1)$ is $\left(w_{0}, w_{1}, \ldots, w_{n}\right)$, where $w_{i}=\tilde{\theta}(i)$. Let $E(n)$ denote the path-connected component of $F\left(\mathbb{R}^{+}, n+1\right)$ that contains the point $(0,1, \ldots, n)$. Let $C E(n)=(E(n) \times[0,1]) /(E(n) \times\{1\})$ be the (unreduced) cone of $E(n)$ and let

$$
\tilde{F}(M, n+1)=F(M, n+1) \cup_{j_{n}} C E(n)
$$

be the unreduced mapping cone of the map $j_{n}$ given by the composite

$$
j_{n}: E(n) \hookrightarrow F\left(\mathbb{R}^{+}, n+1\right) \stackrel{F(\tilde{\theta}, n+1)}{\longrightarrow} F(M, n+1) .
$$

Note that $\left(w_{0}, \ldots, w_{n}\right)$ is identified with $((0,1, \ldots, n), 0) \in C E(n)$. The basepoint of $\tilde{F}(M, n+1)$ is the point given by the image of $E(n) \times\{1\}$. Let

$$
\bar{F}(M, n+1)=\tilde{F}(M, n+1) /((0,1, \ldots, n) \times[0,1])
$$

be the reduced mapping cone of $j_{n}$. The quotient map

$$
q_{n}: \tilde{F}(M, n+1) \longrightarrow \bar{F}(M, n+1)
$$


and the composite

$$
f_{n}: F(M, n+1) \hookrightarrow \tilde{F}(M, n+1) \stackrel{q_{n}}{\longrightarrow} \bar{F}(M, n+1)
$$

are pointed maps. Clearly $q_{n}: \tilde{F}(M, n+1) \rightarrow \bar{F}(M, n+1)$ is a (pointed) homotopy equivalence. Recall that each path-connected component of $F\left(\mathbb{R}^{+}, n+1\right)$ is contractible [53]. The space $E(n)$ is contractible, and so the map

$$
f_{n}: F(M, n+1) \longrightarrow \bar{F}(M, n+1)
$$

is a pointed homotopy equivalence.

Observe that the face $d_{i}: F\left(\mathbb{R}^{+}, n+1\right) \rightarrow F\left(\mathbb{R}^{+}, n\right)$ maps $E(n)$ into $E(n-1)$. This defines a map

$$
\tilde{d}_{i}=d_{i} \cup C d_{i}: F(M, n+1) \cup_{j_{n}} C E(n) \longrightarrow F(M, n) \cup_{j_{n-1}} C E(n-1)
$$

for $0 \leq i \leq n$. The resulting faces $\tilde{d}_{i}: \tilde{F}(M, n+1) \rightarrow \tilde{F}(M, n)$ are pointed maps satisfying $\tilde{d}_{j} \tilde{d}_{i}=\tilde{d}_{i} \tilde{d}_{j+1}$ for $i \leq j$.

Let $A$ be any pointed map. For $0 \leq i \leq n$, define

$$
d_{i}:[A, F(M, n+1)] \rightarrow[A, F(M, n)]
$$

to be the unique function such that the diagram

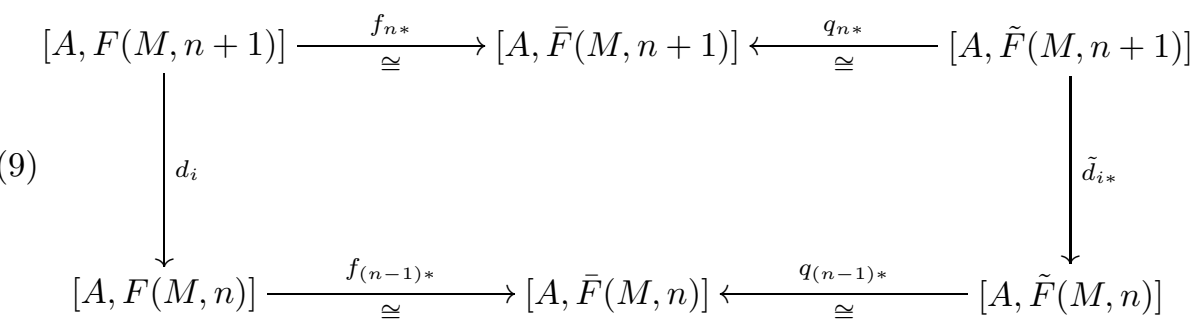

commutes. This proves the following.

Proposition 5.1. Let $M$ be a space with a good basepoint and let $A$ be any pointed space. Then the sequence of sets of (pointed) homotopy classes

$$
\Gamma_{*}(A, M)=\{[A, F(M, n+1)]\}_{n \geq 0}
$$

is a $\Delta$-set under the faces defined above.

Corollary 5.2. Let $M$ be a space with a good basepoint. Then the sequence of groups $\{[A, F(M, n+1)]\}_{n \geq 0}$ is a $\Delta$-group for any cogroup space $A$.

Now let $M$ be a metric space with a steady flow $\theta$. Consider the degeneracy

$$
s_{i}: F(M, n+1) \rightarrow F(M, n+2)
$$

defined in Equation (4). Observe that $s_{i}: F\left(\mathbb{R}^{+}, n+1\right) \rightarrow F\left(\mathbb{R}^{+}, n+2\right)$ maps $E(n)$ into $E(n+1)$. This defines a map

$$
\tilde{s}_{i}=s_{i} \cup C s_{i}: F(M, n+1) \cup_{j_{n}} C E(n) \longrightarrow F(M, n+2) \cup_{j_{n+1}} C E(n+1)
$$

for $0 \leq i \leq n$. The resulting degeneracy $\tilde{s}_{i}: \tilde{F}(M, n+1) \rightarrow \tilde{F}(M, n+1)$ is a pointed map. For $0 \leq i \leq n$, define

$$
s_{i}:[A, F(M, n+1)] \rightarrow[A, F(M, n+2)]
$$


to be the unique function such that the diagram

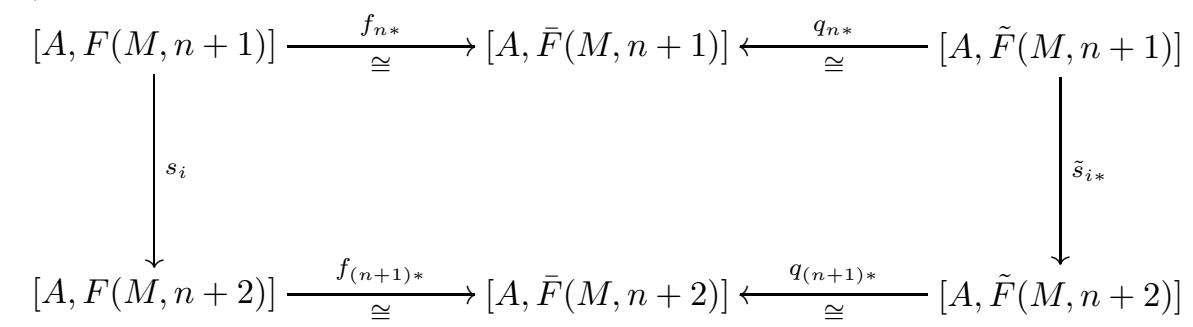

commutes.

Proof of Theorem 1.4, Part (i) is given in Proposition 5.1 and Corollary 5.2 , (ii). The identity $d_{j} d_{i}=d_{i} d_{j+1}$ for $i \leq j$ has been proved in Proposition 5.1. Let

$$
L_{j, i}: F(M, n+1) \times I \rightarrow F(M, n+1)
$$

be the map defined by Equation (5) and let

$$
\tilde{L}_{j, i}: F(M, n+1) \times I \rightarrow F(M, n+3)
$$

be the map defined by Equation (77). The maps $L_{j, i}$ and $\tilde{L}_{j, i}$ induce pointed homotopies

$$
\begin{gathered}
F_{j, i}=L_{j, i} \cup C L_{j, i}:\left(F(M, n+1) \cup_{j_{n}} C E(n)\right) \times I \longrightarrow F(M, n+1) \cup_{j_{n}} C E(n), \\
G_{j, i}=\tilde{L}_{j, i} \cup C \tilde{L}_{j, i}:\left(F(M, n+1) \cup_{j_{n}} C E(n)\right) \times I \longrightarrow F(M, n+3) \cup_{j_{n}} C E(n+2),
\end{gathered}
$$

respectively. By Equations (6) and (8), the simplicial identities hold, up to pointed homotopy given by $F_{j, i}$ and $G_{j, i}$, for the sequence of spaces $\{\tilde{F}(M, n+1)\}_{n \geq 0}$. So the theorem follows.

Proposition 5.3. Let $M$ be a compact, connected oriented differentiable manifold. Then the following statements are equivalent.

(1) For any pointed space $A$, there exist degeneracies on the $\Delta$-set $\Gamma_{*}(A, M)$ such that $\Gamma_{*}(A, M)$ is a simplicial set.

(2) There exist degeneracies on the $\Delta$-set $\Gamma_{*}(M, M)$ such that $\Gamma_{*}(M, M)$ is a simplicial set.

(3) $M$ has nonempty boundary, or has zero Euler characteristic.

(4) $M$ admits a nonvanishing vector field.

(5) There exists a steady flow over $M$.

Proof. The equivalence of Statements (3)-(5) was given in Corollary 3.2.7. Theorem 1.4 shows that $(5) \Rightarrow(1)$. It is immediate that $(1) \Rightarrow(2)$. Suppose that Statement (2) is true. Let $\alpha=s_{0}([\mathrm{id}]) \in \Gamma_{1}(M, M)=[M, F(M, 2)]$ be represented by a map $s: M \rightarrow F(M, 2)$. Since [id] $=d_{0} s_{0} \alpha=d_{1} s_{0} \alpha$, the composite

$$
M \stackrel{s}{\longrightarrow} F(M, 2) \hookrightarrow M^{2}
$$

is homotopic to the diagonal map. According to the second paragraph in the proof of Proposition 3.2.6. Statement (3) now holds. This finishes the proof. 


\section{Proofs of Theorems 1.1, 1.2 And 1.3}

6.1. A simplicial group model for $\Omega S^{2}$. Let $\mathcal{X}$ be a pointed simplicial set. Let $* \in X_{0}$ be the basepoint. The basepoint in $X_{n}$ is $s_{0}^{n} *$. Let $F[\mathcal{X}]_{n}$ be the free group generated by $X_{n}$ subject to the single relation that $s_{0}^{n} *=1$. (Note. By the simplicial identities, $s_{0}^{n}=s_{i_{n}} s_{i_{n-1}} \cdots s_{i_{1}}$ for any sequence $\left(i_{1}, i_{2}, \ldots, i_{n}\right)$ with $0 \leq i_{k} \leq k-1$.) Then we obtain the simplicial group $F[\mathcal{X}]=\left\{F[\mathcal{X}]_{n}\right\}_{n \geq 0}$ with the faces and the degeneracies induced by those of $\mathcal{X}$. The simplicial group $F[\mathcal{X}]$ is called Milnor's free group construction on $\mathcal{X}$. An important property of the construction of $F[\mathcal{X}]$ is as follows.

Theorem 6.1.1 (Milnor [55]). If $X$ is a reduced simplicial set, then the geometric realization $|F[\mathcal{X}]|$ of $F[\mathcal{X}]$ is homotopy equivalent to $\Omega \Sigma|\mathcal{X}|$.

Note. The geometric realization of $F[\mathcal{X}]$ is the group completion of the James construction on $|\mathcal{X}|$. There are natural extensions of the above theorem in case $X$ is not assumed to be reduced. However, those extensions are not given in Milnor's original article.

Now let $S^{1}$ be the simplicial 1-sphere. The elements in $S_{n}^{1}$ can be listed as follows.

$S_{0}^{1}=\{*\}, S_{1}^{1}=\left\{s_{0} *, \sigma\right\}, S_{2}^{1}=\left\{s_{0}^{2} *, s_{0} \sigma, s_{1} \sigma\right\}, S_{3}^{1}=\left\{s_{0}^{3} *, s_{2} s_{1} \sigma, s_{2} s_{0} \sigma, s_{1} s_{0} \sigma\right\}$, and in general $S_{n+1}^{1}=\left\{s_{0}^{n+1} *, x_{0}, \ldots, x_{n}\right\}$, where $x_{j}=s_{n} \cdots \hat{s}_{j} \cdots s_{0} \sigma$. The face

$$
d_{i}: S_{n+1}^{1}=\left\{*, x_{0}, \ldots, x_{n}\right\} \longrightarrow S_{n}^{1}=\left\{*, x_{0}, \ldots, x_{n-1}\right\}
$$

is given by $d_{i} s_{0}^{n+1} *=s_{0}^{n} *$ and

$$
d_{i} x_{j}=d_{i} s_{n} \cdots \hat{s}_{j} \cdots s_{0} \sigma= \begin{cases}s_{0}^{n} * & \text { if } j=i=0 \quad \text { or } \quad i=j+1=n+1, \\ x_{j} & \text { if } j<i \\ x_{j-1} & \text { if } j \geq i .\end{cases}
$$

Similarly,

$$
s_{i} x_{j}=s_{i} s_{n} \cdots \hat{s}_{j} \cdots s_{0} \sigma=\left\{\begin{array}{lll}
x_{j} & \text { if } & j<i \\
x_{j+1} & \text { if } & j \geq i .
\end{array}\right.
$$

Consider the special case of Milnor's free group construction for $S^{1}, F\left[S^{1}\right]$. According to Theorem 6.1.1, $F\left[S^{1}\right]$ is a simplicial group model for $\Omega S^{2}$. As a sequence of groups, the group $F\left[S^{1}\right]_{n}$ is the free group of rank $n$ generated by $x_{0}, x_{1}, \ldots, x_{n-1}$ with faces as above. Tietze transformations may be used to change the basis of the free group $F\left[S^{1}\right]_{n+1}$, so as to reformulate the faces $d_{i}$ in a canonical way.

Let $y_{0}=x_{0} x_{1}^{-1}, \ldots, y_{n-1}=x_{n-1} x_{n}^{-1}$ and $y_{n}=x_{n}$ in $F\left[S^{1}\right]_{n+1}$. Clearly $\left\{y_{0}, y_{1}, \ldots, y_{n}\right\}$ is a set of free generators for $F\left[S^{1}\right]_{n+1}$ with

$$
d_{i} y_{j}(0 \leq i \leq n+1,-1 \leq j \leq n)
$$

given by

$$
d_{i} y_{j}=d_{i}\left(x_{j} x_{j+1}^{-1}\right)=\left\{\begin{array}{ll}
y_{j} & \text { if } j<i-1, \\
1 & \text { if } j=i-1, \\
y_{j-1} & \text { if } j \geq i,
\end{array} \quad s_{i} y_{j}= \begin{cases}y_{j} & \text { if } j<i-1, \\
y_{j} y_{j+1} & \text { if } j=i-1, \\
y_{j+1} & \text { if } j \geq i\end{cases}\right.
$$

where $y_{-1}=\left(y_{0} y_{1} \cdots y_{n-1}\right)^{-1}$ and in this formula $x_{n+1}=1$. Under the generating system of $y_{j}$ 's, the faces $d_{i}$ with $i>0$ are projection maps in the sense that $d_{i}$ sends $y_{i-1}$ to 1 and other generators to the generators for $F\left[S^{1}\right]_{n}$ so as to retain 
the order. The first face $d_{0}$ differs from the others as $d_{0}$ sends $y_{0}$ to the product element $\left(y_{0} y_{1} \cdots y_{n-1}\right)^{-1}$ and each other generator $y_{j}$ to $y_{j-1}$ for $F\left[S^{1}\right]_{n}$.

To describe all faces $d_{i}$ systematically in terms of projections, consider the free group of rank $n$ in the following way. Let $\hat{F}_{n+1}$ be the quotient of the free group $F\left(z_{0}, z_{1}, \ldots, z_{n}\right)$ subject to the single relation

$$
z_{0} z_{1} \cdots z_{n}=1 .
$$

Let $\hat{z}_{j}$ be the image of $z_{j}$ in $\hat{F}_{n+1}$. The group $\hat{F}_{n+1}$ is written $\hat{F}\left(\hat{z}_{0}, \hat{z}_{1}, \ldots, \hat{z}_{n}\right)$ in case the generators $\hat{z}_{j}$ are used. Clearly

$$
\hat{F}_{n+1} \cong F\left(\hat{z}_{0}, \hat{z}_{1}, \ldots, \hat{z}_{n-1}\right)
$$

is a free group of rank $n$. Define the faces $d_{i}$ and degeneracies $s_{i}$ on $\left\{\hat{F}_{n+1}\right\}_{n \geq 0}$ as follows:

$$
d_{i} \hat{z}_{j}=\left\{\begin{array}{ll}
\hat{z}_{j} & \text { if } \quad j<i, \\
1 & \text { if } \quad j=i, \\
\hat{z}_{j-1} & \text { if } \quad j>i,
\end{array} \quad s_{i} \hat{z}_{j}=\left\{\begin{array}{lll}
\hat{z}_{j} & \text { if } \quad j<i, \\
\hat{z}_{i} \hat{z}_{i+1} & \text { if } \quad j=i, \\
\hat{z}_{j+1} & \text { if } \quad j>i .
\end{array}\right.\right.
$$

It is straightforward to check that the sequence of groups $\hat{F}=\left\{\hat{F}_{n+1}\right\}_{n \geq 0}$ is a simplicial group under $d_{i}$ and $s_{i}$ defined as above. Let

$$
\phi_{n}: \hat{F}_{n+1} \rightarrow F\left[S^{1}\right]_{n}
$$

be the group homomorphism given by $\phi_{n}\left(\hat{z}_{0}\right)=\left(y_{0} y_{1} \cdots y_{n-1}\right)^{-1}$ and $\phi_{n}\left(\hat{z}_{j}\right)=y_{j-1}$ for $1 \leq j \leq n$. It is routine to check part (1) of the following result. Then Theorem 6.1.1 has part (2) as an application.

Proposition 6.1.2. Let $\hat{F}$ be the simplicial group defined above. Then

(1) $\phi=\left\{\phi_{n}\right\}: \hat{F} \rightarrow F\left[S^{1}\right]$ is an isomorphism of simplicial groups; and

(2) the geometric realization of $\hat{F}$ is homotopy equivalent to $\Omega S^{2}$.

6.2. Proof of Theorem 1.1. In this subsection, we prove Theorem 1.1, while the computations of low-dimensional Brunnian braids over $S^{2}$ will be given in Section 7 Let $\mathcal{E}=\left\{F\left(S^{2}, n+1\right)\right\}_{n \geq 0}$. Then $\mathcal{E}$ is a $\Delta$-bundle with $E_{n}=F\left(S^{2}, n+1\right)$ pathconnected and $E_{0}=S^{2}$ simply-connected. So from Definition 4.2.3, there is a fibration

$$
(\tilde{\Omega} \mathcal{E})_{n} \longrightarrow F\left(S^{2}, n+2\right) \stackrel{d_{0}}{\longrightarrow} F\left(S^{2}, n+1\right) .
$$

Part (a) of the next lemma follows at once by comparing with the canonical fibration (with $Q_{n+1}=\left\{q_{0}, \ldots, q_{n}\right\}$ )

$$
S^{2}-Q_{n+1} \longrightarrow F\left(S^{2}, n+2\right) \stackrel{d_{0}}{\longrightarrow} F\left(S^{2}, n+1\right) .
$$

Lemma 6.2.1. (a) For all $n$ there is a homotopy equivalence $(\tilde{\Omega} \mathcal{E})_{n} \simeq S^{2}-Q_{n+1}$.

(b) There are isomorphisms of $\Delta$-groups

$$
(\tilde{\Omega} \mathcal{E})^{\pi_{1}} \cong \hat{F} \cong F\left[S^{1}\right] .
$$

Proof. The face map $d_{i}: \tilde{\Omega} E_{n} \rightarrow \tilde{\Omega} E_{n-1}$ is the inclusion

$$
S^{2}-Q_{n+1} \subseteq S^{2}-\left\{q_{0}, \ldots, q_{i-1}, q_{i+1}, \ldots, q_{n}\right\} .
$$

Thus $\pi_{1}\left((\tilde{\Omega} \mathcal{E})_{n}\right)=\hat{F}_{n+1}$, and it is easy to see that the respective faces agree. Therefore, by Part (1) of Proposition 6.1.2, there is an isomorphism of $\Delta$-groups

$$
F\left[S^{1}\right] \cong(\tilde{\Omega} \mathcal{E})^{\pi_{1}} .
$$


Part (2) of Proposition 6.1.2 has the following consequence.

Corollary 6.2.2. There are isomorphisms

$$
\pi_{n}\left((\tilde{\Omega} \mathcal{E})^{\pi_{1}}\right) \cong \pi_{n}\left(F\left[S^{1}\right]\right) \cong \pi_{n+1}\left(S^{2}\right)
$$

for any $n$.

Proof of Theorem 1.1. Observe that, by definition,

$$
\mathcal{E}^{\pi_{1}}=\mathcal{F}\left(S^{2}\right)^{\pi_{1}} .
$$

Recall from Corollary 2.3 that $\pi_{1}\left(F\left(S^{2}, 3\right)\right)=\mathbb{Z} / 2$ and also $\pi_{2}\left(F\left(S^{2}, n\right)\right)=0$ for $n \geq 3$. From the exact sequence of Proposition 4.2.4.

$$
\pi_{2}\left(F\left(S^{2}, n+1\right)\right) \longrightarrow \pi_{1}\left((\tilde{\Omega} \mathcal{E})_{n}\right) \longrightarrow \Omega\left(\mathcal{E}^{\pi_{1}}\right)_{n} \longrightarrow 1,
$$

we have

$$
\pi_{1}\left((\tilde{\Omega} \mathcal{E})_{n}\right) \cong \Omega\left(\mathcal{E}^{\pi_{1}}\right)_{n}
$$

for $n \geq 2$. Thus, via Part (3) of Proposition 4.2.1, for $n \geq 3$,

$$
\pi_{n+1}\left(\mathcal{F}\left(S^{2}\right)^{\pi_{1}}\right)=\pi_{n+1}\left(\mathcal{E}^{\pi_{1}}\right)=\pi_{n}\left(\Omega\left(\mathcal{E}^{\pi_{1}}\right)\right) \cong \pi_{n}\left((\tilde{\Omega} \mathcal{E})^{\pi_{1}}\right) \cong \pi_{n+1}\left(S^{2}\right) .
$$

This completes the proof.

Theorem 6.2.3. The group of Brunnian braids $\operatorname{Brun}_{n}\left(S^{2}\right)$ is isomorphic to the group of Moore cycles $\mathrm{Z}_{n-2} F\left[S^{1}\right]$ for $n \geq 5$.

Proof. From the definitions,

$$
\operatorname{Brun}_{n}\left(S^{2}\right)=\mathrm{Z}_{n-1} \mathcal{F}\left(S^{2}\right)^{\pi_{1}}=\mathrm{Z}_{n-2} \Omega\left(\mathcal{F}\left(S^{2}\right)^{\pi_{1}}\right),
$$

while the above proof also gives

$$
\mathrm{Z}_{n-2} \Omega\left(\mathcal{F}\left(S^{2}\right)^{\pi_{1}}\right) \cong \mathrm{Z}_{n-2}\left(\tilde{\Omega} \mathcal{F}\left(S^{2}\right)\right)^{\pi_{1}} \cong \mathrm{Z}_{n-2} F\left[S^{1}\right]
$$

for $n-2 \geq 3$.

6.3. Artin's braids. In this subsection, Artin's braid groups are considered. The main reference is Birman's book [6]. Let $B_{n+1}=\pi_{1}\left(B\left(D^{2}, n+1\right)\right)$ denote the Artin braid group and let $P_{n+1}=\pi_{1}\left(F\left(D^{2}, n+1\right)\right) \subseteq B_{n+1}$ denote the Artin pure braid group.

Let $D^{2}$ be the unit disc and let $Q_{n+1}=\left\{q_{0}, q_{1}, \ldots, q_{n}\right\}$ be a set of distinct fixed points of $D^{2}-\partial D^{2}$. Then $\pi_{1}\left(D^{2}-Q_{n+1}\right)$ is a free group $F_{n+1}$ of rank $n+1$. Let $z_{0}, z_{1}, \ldots, z_{n}$ be a basis for $\pi_{1}\left(D^{2}-Q_{n+1}\right)$, where $z_{i}$ is represented by a simple loop that encloses the point $q_{i}$, but no point $q_{j}$ for $j \neq i$. According to [6. Theorem 1.10], the braid group $B_{n+1}$ is isomorphic to the group of automorphisms of $\pi_{1}\left(D^{2}-Q_{n+1}\right)$ induced by the self-homeomorphisms of $D^{2}-Q_{n+1}$ that keep the boundary of $D^{2}$ fixed pointwise. (This result is due to Artin and can be regarded as another definition of the group $B_{n+1}$.)

According to [6. pp. 33-34], this isomorphism can be described as follows. Let $h$ be a self-homeomorphism of $D^{2}-Q_{n+1}$. Then $h$ has a unique extension $\bar{h}$ to $D^{2}$ which permutes the points of $Q_{n+1}$. The map $\bar{h}$ is isotopic to the identity map of $D^{2}$ relative to the boundary. Let $F^{h}: D^{2} \times I \rightarrow D^{2}$ be such an isotopy, with $F_{0}^{h}=\operatorname{id}_{D^{2}}$ and $F_{1}^{h}=\bar{h}$. The image $\beta=\beta_{h}$ of $Q_{n+1} \times I$ under $F^{h}$ is a geometric braid of $n+1$ strings over $D^{2}$.

Let $Q_{n+1, i}=Q_{n+1}-\left\{q_{i}\right\}$, and define $\mu_{\beta} \in S_{n+1}$ by $q_{\mu_{\beta}(j)}=\bar{h}\left(q_{j}\right)$ for $0 \leq j \leq n$. Recall that the simplicial structure on $\left\{B_{n+1}\right\}$ is given by deleting and doubling the 
strings. More precisely, $d_{i} \beta$ is the braid of $n$ strings obtained by taking the image of $Q_{n+1, i}$ under $F^{h}$ in $D^{2} \times I$, and $s_{i} \beta$ is the braid of $n+2$ strings obtained by taking the image of $Q_{n+1} \cup\left\{q_{i}^{\prime}\right\}$ under $F^{h}$ in $D^{2} \times I$, where $q_{i}^{\prime}$ is a point sufficiently close to but different from $q_{i}$. (See Subsection 3.2 for the choice of the point $q_{i}^{\prime}$.) Observe that the geometric braids $d_{i} \beta$ and $s_{i} \beta$ are induced by the restricted homeomorphisms $\bar{h}: D^{2}-Q_{n+1, i} \rightarrow D^{2}-Q_{n+1, \mu_{\beta}(i)}$ and

$$
\bar{h}: D^{2}-\left(Q_{n+1} \cup\left\{q_{i}^{\prime}\right\}\right) \rightarrow D^{2}-\left(Q_{n+1} \cup\left\{\bar{h}\left(q_{i}^{\prime}\right)\right\}\right),
$$

respectively. (Note. According to Subsection 3.2, the point $q_{i}^{\prime}$ is chosen in a good way such that the string from $q_{i}^{\prime}$ to $\bar{h}\left(q_{i}^{\prime}\right)$ lies inside a small tubular neighborhood $V_{i}$ of the $i$-th string from $q_{i}$ to $\bar{h}\left(q_{i}\right)$ and the string from $q_{j}$ to $\bar{h}\left(q_{j}\right)$ lies outside of $V_{i}$ for $j \neq i$.) Define the faces $d_{i}$ and the degeneracies $s_{i}$ on $\left\{F_{n+1}\right\}_{n \geq 0}$ as in equation (12) (with $\hat{z}$ replaced by $z$ ). The next lemma follows from a routine check.

Lemma 6.3.1. Let $\beta \in P_{n+1}$ be a pure braid, regarded as above as an automorphism of the free group $F_{n+1} \cong \pi_{1}\left(D^{2}-Q_{n+1}\right)$. Then there is a commutative diagram

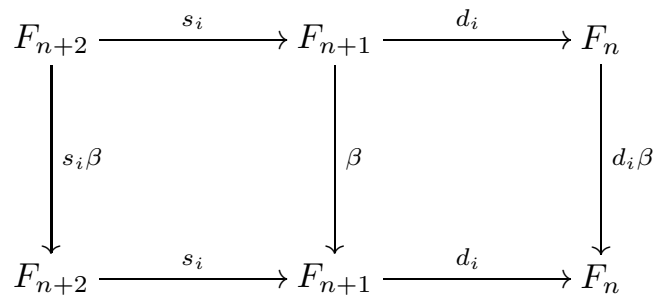

for $0 \leq i \leq n$.

Recall that the group $B_{n+1}$ admits a representation with generators $\sigma_{0}, \sigma_{1}, \ldots$, $\sigma_{n-1}$ and defining relations

(1) $\sigma_{i} \sigma_{j}=\sigma_{j} \sigma_{i}$ for $|i-j| \geq 2$ and $0 \leq i, j \leq n-1$, and

(2) $\sigma_{i} \sigma_{i+1} \sigma_{i}=\sigma_{i+1} \sigma_{i} \sigma_{i+1}$ for $0 \leq i \leq n-2$.

(Note that our labelling system starts with 0 .) As a geometric braid, $\sigma_{i}$ is the canonical $i$-th elementary braid of $n+1$ strings pictured below, which twists the positions $i$ and $i+1$ once and puts trivial strings on the remaining positions.

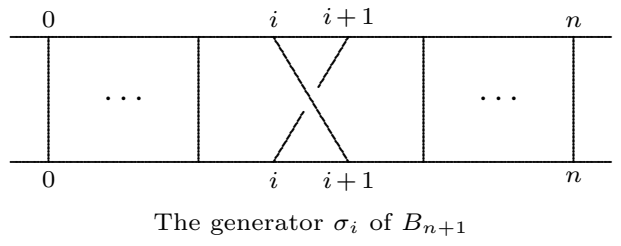

The action of $\sigma_{i}$ on $F_{n+1}=\pi_{1}\left(D^{2}-Q_{n+1}\right)$ is given as follows:

$$
\sigma_{i}\left(z_{i}\right)=z_{i+1}, \sigma_{i}\left(z_{i+1}\right)=z_{i+1}^{-1} z_{i} z_{i+1} \text { and } \sigma_{i}\left(z_{j}\right)=z_{j} \text { for } j \neq i, i+1 .
$$

The classical Artin theorem [2] describes $B_{n+1}$ as a subgroup of $\operatorname{Aut}\left(F_{n+1}\right)$. 
Theorem 6.3.2. [6, Theorem 1.9] Let $\beta$ be an endomorphism of $F_{n+1}$. Then $\beta$ lies in $B_{n+1} \subseteq \operatorname{Aut}\left(F_{n+1}\right)$ if and only if $\beta$ satisfies two conditions:

(1) $\beta\left(z_{i}\right)=A_{i}^{-1} z_{\sigma(i)} A_{i}, 0 \leq i \leq n$, for some permutation $\sigma \in S_{n+1}$ and words $A_{i} \in F_{n+1}$, and

(2) $\beta\left(z_{0} z_{1} \cdots z_{n}\right)=z_{0} z_{1} \cdots z_{n}$.

In fact, in the above result $\sigma$ can be taken as $\mu_{\beta}$. The center $\mathcal{Z}\left(B_{n+1}\right)$ of $B_{n+1}$ is as follows.

Lemma 6.3.3 (Chow 20]). If $n \geq 2$, then $\mathcal{Z}\left(B_{n+1}\right)=\mathcal{Z}\left(P_{n+1}\right)$ is the infinite cyclic subgroup generated by $\left(\sigma_{0} \sigma_{1} \cdots \sigma_{n-1}\right)^{n+1}$.

(Note. If $n=1$, then $\mathcal{Z}\left(P_{2}\right)=P_{2} \cong \mathbb{Z}$ is generated by $\sigma_{0}^{2}$. Thus $\mathcal{Z}\left(P_{n+1}\right)$ is in fact the subgroup generated by $\left(\sigma_{0} \sigma_{1} \cdots \sigma_{n-1}\right)^{n+1}$ for each $n$.) Let $\hat{F}_{n+1}$ (as before) denote the group generated by $\hat{z}_{0}, \ldots, \hat{z}_{n}$, with the single relation $\hat{z}_{0} \hat{z}_{1} \cdots \hat{z}_{n}=1$. It is a free group of rank $n$ with a basis $\hat{z}_{0}, \ldots, \hat{z}_{n-1}$. By the second condition of Theorem 6.3.2, the product element $z_{0} z_{1} \cdots z_{n}$ is a fixed point of the $B_{n+1}$-action, and so the action of $B_{n+1}$ on $F_{n+1}$ factors through the quotient group $\hat{F}_{n+1}$. The resulting representation $B_{n+1} \rightarrow \operatorname{Aut}\left(\hat{F}_{n+1}\right)$ is called the reduced Artin representation. Let $\phi: P_{n+1} \subseteq B_{n+1} \rightarrow \operatorname{Aut}\left(\hat{F}_{n+1}\right)$ be the reduced Artin representation of the pure braid group $P_{n+1}$. This representation is not faithful.

Lemma 6.3.4. [6, Lemma 3.17.2] The kernel of $\phi: P_{n+1} \rightarrow \operatorname{Aut}\left(\hat{F}_{n+1}\right)$ is the center $\mathcal{Z}\left(P_{n+1}\right)$.

Lemma 6.3.5. Let $\beta \in B_{n+1}$ be a braid. If the reduced Artin representation of $\beta$ is an inner automorphism of $\hat{F}_{n+1}$, then $\beta$ is a pure braid.

Proof. The assertion follows immediately from the commutative diagram

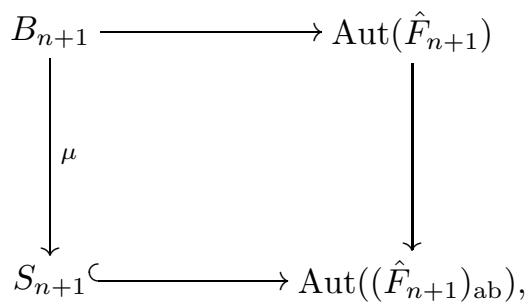

where the abelianization $\left(\hat{F}_{n+1}\right)$ ab of $\hat{F}_{n+1}$ is the quotient of $\mathbb{Z}^{n+1}$ with the reduced basis $\left\{\hat{e}_{0}, \ldots, \hat{e}_{n}\right\}$ subject to the relation $\hat{e}_{0}+\hat{e}_{1}+\cdots+\hat{e}_{n}=0$, and $S_{n+1}$ acts on $\left(\hat{F}_{n+1}\right)_{\text {ab }}$ by permuting the reduced basis.

Let $R_{n+1}=\phi^{-1}\left(\operatorname{Inn}\left(\hat{F}_{n+1}\right)\right)$ be the subgroup of $P_{n+1}$ consisting of those braids $\beta$ whose reduced Artin representation is an inner automorphism of $\hat{F}_{n+1}$. It is clear that $R_{n+1}$ is a normal subgroup of $B_{n+1}$.

Proposition 6.3.6. Let $R=\left\{R_{n+1}\right\}_{n \geq 0}$ and let $C=\left\{\mathcal{Z}\left(P_{n+1}\right)\right\}_{n \geq 0}$. Then the following hold.

(1) Both $C$ and $R$ are $\Delta$-subgroups of $\mathcal{P}=\left\{P_{n+1}\right\}_{n \geq 0}$.

(2) The Moore chains of $C$ are given by $N_{1} C \cong \mathbb{Z}$ and $N_{j} C=1$ for $j \neq 1$.

(3) There are isomorphisms $N_{n} R \cong N_{n} F\left[S^{1}\right]$ and $\mathrm{Z}_{n} R \cong \mathrm{Z}_{n} F\left[S^{1}\right]$ for $n \geq 3$. 
Proof. Because the face maps are epimorphisms, they send centers to centers; and so $C$ is a $\Delta$-subgroup of $\mathcal{P}$. By Lemma 6.3.1 there is a commutative diagram

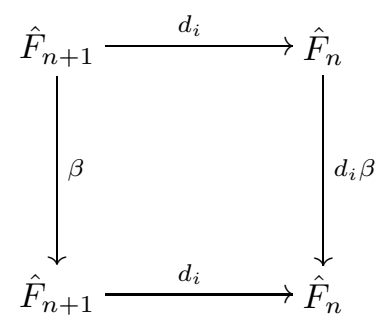

for $\beta \in P_{n+1}$ and $0 \leq i \leq n$. Let $\beta \in R_{n+1}$. Then there is a word $w \in \hat{F}_{n+1}$ such that $\beta(z)=w^{-1} z w$ for any $z \in \hat{F}_{n+1}$. Thus

$$
\left(d_{i} \beta\right)\left(d_{i}(z)\right)=\left(d_{i}(w)\right)^{-1} d_{i}(z) d_{i}(w) .
$$

Since $d_{i}: \hat{F}_{n+1} \rightarrow \hat{F}_{n}$ is onto, the reduced Artin representation of $d_{i} \beta$ is the inner automorphism induced by $d_{i} w$. Thus $d_{i} \beta \in R_{n}$ for each $0 \leq i \leq n$ and assertion (1) follows. Now determine the Moore chains of $C$ and $R$. Since $\mathcal{B}\left(D^{2}\right)^{\pi_{1}}=\left\{B_{n+1}\right\}_{n \geq 0}$ is a crossed simplicial group, from (3.1),

$$
d_{i}\left(x^{k}\right)=d_{i}(x) d_{i \cdot \mu_{x}}(x) \cdots d_{i \cdot \mu_{x}^{k-1}}(x)
$$

for $x \in \mathcal{B}\left(D^{2}\right)^{\pi_{1}}$ and $k \geq 1$. Let $\beta_{n}=\sigma_{0} \sigma_{1} \cdots \sigma_{n-1} \in\left(\mathcal{B}\left(D^{2}\right)^{\pi_{1}}\right)_{n}$. Then $d_{0} \beta_{n}=1$ and $d_{j} \beta_{n}=\beta_{n-1}$ for $j>0$. It follows that

$$
d_{i}\left(\beta_{n}^{n+1}\right)=d_{i}\left(\beta_{n}\right) d_{i \cdot \rho}\left(\beta_{n}\right) \cdots d_{i \cdot \rho^{n}}\left(\beta_{n}\right)=\beta_{n-1}^{n}
$$

because $\rho=\mu_{\beta_{n}}$ is a cyclic permutation of order $n+1$. Assertion (2) follows. (3). By [6, Lemmas 3.17.1 and 3.17.2], there is a split short exact sequence

$$
1 \longrightarrow \mathcal{Z}\left(P_{n+1}\right) \longrightarrow R_{n+1} \stackrel{\phi}{\longrightarrow} \operatorname{Inn}\left(\hat{F}_{n+1}\right) \longrightarrow 1 \text {. }
$$

Thus

$$
\operatorname{Inn}(\hat{F})=\left\{\operatorname{Inn}\left(\hat{F}_{n+1}\right)\right\}_{n \geq 0}
$$

is a $\Delta$-quotient group of $R$. Let $\theta: \hat{F}_{n+1} \rightarrow \operatorname{Inn}\left(\hat{F}_{n+1}\right)_{n \geq 0}$ be the map defined by $\theta(w)(z)=w^{-1} z w$. By equation (13), the map

$$
\theta: F\left[S^{1}\right]=\hat{F} \rightarrow \operatorname{Inn}(\hat{F})
$$

is a morphism of $\Delta$-groups. Since $\theta: \hat{F}_{n+1} \rightarrow \operatorname{Inn}\left(\hat{F}_{n+1}\right)$ is an isomorphism for $n \geq 2$, it follows that $\theta_{*}: N_{n} F\left[S^{1}\right] \rightarrow N_{n} \operatorname{Inn}(\hat{F})$ and $\theta_{*}: \mathrm{Z}_{n} F\left(S^{1}\right) \rightarrow \mathrm{Z}_{n} \operatorname{Inn}(\hat{F})$ are isomorphisms for $n \geq 3$. It remains to show that $\phi_{*}: N_{n} R \rightarrow N_{n} \operatorname{Inn}(\hat{F})$ and $\phi_{*}: \mathrm{Z}_{n} R \rightarrow \mathrm{Z}_{n} \operatorname{Inn}(\hat{F})$ are isomorphisms for $n \geq 3$. By the exact sequence

$$
1 \longrightarrow N C \longrightarrow N R \stackrel{\phi_{*}}{\longrightarrow} N \operatorname{Inn}(\hat{F}),
$$

the map $\phi_{*}: N_{n} R \rightarrow N_{n} \operatorname{Inn}(\hat{F})$ is a monomorphism for $n \geq 2$ because $N_{n} C=1$ for $n \geq 2$. Let $\bar{\beta} \in N_{n} \operatorname{Inn}(\hat{F})$ with $n \geq 3$ and let $\beta$ be an element in $R_{n+1}$ such that $\phi(\beta)=\bar{\beta}$. Let $\alpha_{n}=\beta_{n}^{n+1}$ denote the generator of the infinite cyclic group $\mathcal{Z}\left(P_{n+1}\right)=C_{n}$ for $n \geq 1$. Since $d_{i} \bar{\beta}=1$ for $i>0$, there exist integers $k_{i}$ such that

$$
d_{i} \beta=\alpha_{n-1}^{k_{i}}
$$

for $1 \leq i \leq n$. For each $1 \leq j<i$, observe that

$$
\alpha_{n-2}^{k_{i}}=d_{j} \alpha_{n-1}^{k_{i}}=d_{j} d_{i} \beta=d_{i-1} d_{j} \beta=d_{i-1} \alpha_{n-1}^{k_{j}}=\alpha_{n-2}^{k_{j}} .
$$


It follows that $k_{1}=k_{2}=\cdots=k_{n}$. Let $\tilde{\beta}=\alpha_{n}^{-k_{1}} \beta$. Then $d_{i} \tilde{\beta}=1$ for $i>0$ or $\tilde{\beta} \in N_{n} R$ with $\phi(\tilde{\beta})=\bar{\beta}$. Thus $N_{n} R \rightarrow N_{n} \operatorname{Inn}(\hat{F})$ is an epimorphism for $n \geq 3$. Finally, since $\phi_{*}: N_{n} R \rightarrow N_{n} \operatorname{Inn}(\hat{F})$ is an isomorphism for $n \geq 3$ and a monomorphism for $n=2, \phi_{*}: \mathrm{Z}_{n} R \rightarrow \mathrm{Z}_{n} \operatorname{Inn}(\hat{F})$ is an isomorphism for $n \geq 3$. The proof is complete.

The next corollary is an immediate consequence of Theorem 6.2.3 and Proposition 6.1 .2

Corollary 6.3.7. There are isomorphisms of groups

$$
\mathrm{Z}_{n} R \cong \mathrm{Z}_{n} F\left[S^{1}\right] \cong \operatorname{Brun}_{n+2}\left(S^{2}\right) \quad \text { and } \quad \pi_{n}(R) \cong \pi_{n}\left(F\left[S^{1}\right]\right)=\pi_{n+1}\left(S^{2}\right)
$$

for $n \geq 3$.

Let $S_{+}^{2}$ denote the upper hemisphere. The canonical inclusion $D^{2} \approx S_{+}^{2} \subseteq S^{2}$ induces a map $f: B\left(D^{2}, n+1\right) \rightarrow B\left(S^{2}, n+1\right)$ and so a group homomorphism $f_{*}: \pi_{1}\left(B\left(D^{2}, n+1\right)\right) \rightarrow \pi_{1}\left(B\left(S^{2}, n+1\right)\right)$.

Lemma 6.3.8 (Fadell and Van Buskirk [27]). The group homomorphism

$$
f_{*}: B_{n+1}=\pi_{1}\left(B\left(D^{2}, n+1\right)\right) \rightarrow \pi_{1}\left(B\left(S^{2}, n+1\right)\right)
$$

is an epimorphism, and the kernel $\tilde{R}_{n+1}$ of $f_{*}$ is the normal subgroup of $B_{n+1}$ generated by the single element $\nu_{n}=\sigma_{0} \sigma_{1} \cdots \sigma_{n-2} \sigma_{n-1}^{2} \sigma_{n-2} \cdots \sigma_{0}$.

Notice that

$$
\nu_{n}\left(z_{0}\right)=\left(z_{0} z_{1} z_{2} \cdots z_{n}\right) z_{0}\left(z_{0} z_{1} z_{2} \cdots z_{n}\right)^{-1}
$$

and

$$
\nu_{n}\left(z_{i}\right)=\left(z_{1} z_{2} \cdots z_{n}\right)^{-1} z_{0}^{-1}\left(z_{1} z_{2} \cdots z_{n}\right) z_{i}\left(z_{1} z_{2} \cdots z_{n}\right)^{-1} z_{0}\left(z_{1} z_{2} \cdots z_{n}\right)
$$

for $i>0$. Thus the reduced Artin representation of $\nu_{n}$ is the inner automorphism of $\hat{F}_{n+1}$ induced by $\hat{z}_{0}$ and therefore lies in $R_{n+1}$. Since $R_{n+1}$ is a normal subgroup of $B_{n+1}$, so $\tilde{R}_{n+1}$ is a subgroup of $R_{n+1}$. The following lemma is asserted as a footnote in [6, p. 35]. A short proof is given next.

Lemma 6.3.9. The composite

$$
\tilde{\phi}: \tilde{R}_{n+1} \hookrightarrow R_{n+1} \stackrel{\phi}{\longrightarrow} \operatorname{Inn}\left(\hat{F}_{n+1}\right)
$$

is an epimorphism.

Proof. Observe that the image

$$
\operatorname{Im}\left(\tilde{\phi}: \tilde{R}_{n+1} \rightarrow \operatorname{Inn}\left(\hat{F}_{n+1}\right)\right)
$$

is the subgroup of $\operatorname{Inn}\left(\hat{F}_{n+1}\right)$ generated by inner automorphisms induced by the words $\beta\left(\hat{z}_{0}\right)$ for $\beta \in B_{n+1}$. The assertion follows from the fact that

$$
\sigma_{i-1} \cdots \sigma_{0}\left(\hat{z}_{0}\right)=\hat{z}_{i}
$$

for $1 \leq i \leq n$.

Lemma 6.3.10 (Gillette and Van Buskirk [32]). The center of $\pi_{1}\left(B\left(S^{2}, n+1\right)\right)$ is the subgroup of order 2 generated by the element

$$
f_{*}\left(\left(\sigma_{0} \sigma_{1} \cdots \sigma_{n-1}\right)^{n+1}\right)
$$

for $n \geq 2$. 
Let $\tilde{R}=\left\{\tilde{R}_{n+1}\right\}_{n \geq 0}$ and let $\tilde{C}=C \cap \tilde{R}$. By Lemma 6.3.9, there is a commutative diagram of short exact sequences of $\Delta$-groups

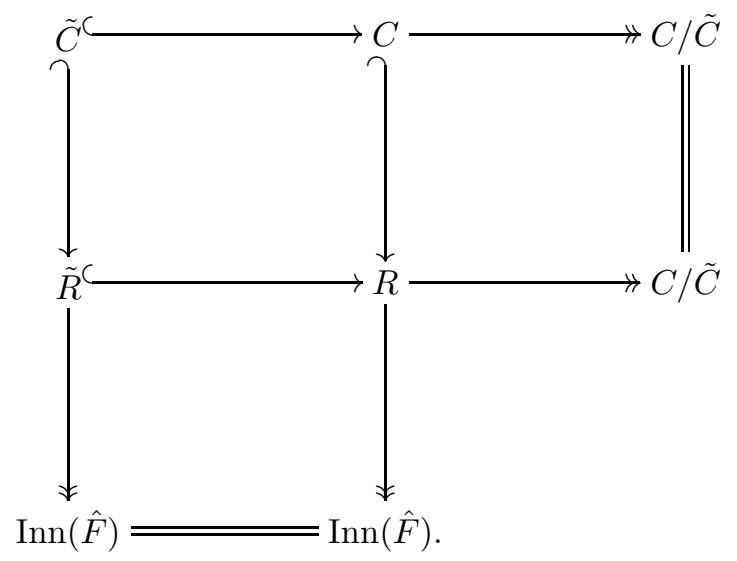

From Lemma 6.3.10, notice that $(C / \tilde{C})_{n}=1$ for $n \leq 1$ and $\mathbb{Z} / 2$ for $n \geq 2$. By Proposition 6.3.6 and Corollary 6.3.7, we have the following.

Proposition 6.3.11. There are isomorphisms of groups

$$
N_{n} \tilde{R} \cong N_{n}\left(F\left[S^{1}\right]\right), \quad \mathrm{Z}_{n} \tilde{R} \cong \mathrm{Z}_{n}\left(F\left[S^{1}\right]\right) \quad \text { and } \quad \pi_{n}(\tilde{R}) \cong \pi_{n}\left(F\left[S^{1}\right]\right)=\pi_{n+1}\left(S^{2}\right)
$$

for $n \geq 3$.

6.4. Proof of Theorem 1.2, The terminology of Subsection 6.3 continues here. Recall that $\mathcal{F}(M)^{\pi_{1}}=\left\{\pi_{1}(F(M, n+1))\right\}_{n \geq 0}$ and that

$$
f: B\left(D^{2}, n+1\right) \longrightarrow B\left(S^{2}, n+1\right)
$$

is the canonical inclusion. Later, in Proposition 7.2.2, $f_{*}\left(\sigma_{i}\right)$ is written as $\delta_{i}$.

Proof of Theorem 1.2, By Theorem 3.2.12, $\mathcal{P}=\mathcal{F}\left(D^{2}\right)^{\pi_{1}}$ is a simplicial group, while by Lemma 6.3.8 $f_{*}: \mathcal{P} \rightarrow \mathcal{F}\left(S^{2}\right)^{\pi_{1}}$ is an epimorphism of $\Delta$-groups. Thus there is a left exact sequence of Moore chains

$$
1 \longrightarrow N \tilde{R} \longrightarrow N \mathcal{P} \stackrel{N f_{*}}{\longrightarrow} N \mathcal{F}\left(S^{2}\right)^{\pi_{1}} .
$$

It will be checked next that

$$
N f_{*}: N_{n} \mathcal{P} \longrightarrow N_{n} \mathcal{F}\left(S^{2}\right)^{\pi_{1}}
$$

is an epimorphism for each $n$.

First, a check for the cases $n=0,1,2$ is given. Recall that $N_{0} \mathcal{F}\left(S^{2}\right)^{\pi_{1}}=1$, $N_{1} \mathcal{F}\left(S^{2}\right)^{\pi_{1}}=1$ and $N_{2} \mathcal{F}\left(S^{2}\right)^{\pi_{1}}=\pi_{1}\left(F\left(S^{2}, 3\right)\right)=\mathbb{Z} / 2$ generated by $\left(\delta_{0} \delta_{1}\right)^{3}=\delta_{1}^{2}$. Observe that $d_{1} \sigma_{1}^{2}=d_{2} \sigma_{1}^{2}=1$. Thus $\sigma_{1}^{2} \in N_{2} \mathcal{P}$ with $f_{*}\left(\sigma_{1}^{2}\right)=\left(\delta_{0} \delta_{1}\right)^{3}$. So the statement holds for $n \leq 2$.

Next, assume that $n \geq 3$. Let $\bar{\beta} \in N_{n} \mathcal{F}\left(S^{2}\right)^{\pi_{1}}$ and let $\beta \in \mathcal{P}_{n}$ be such that $f_{*}(\beta)=\bar{\beta}$. Then

$$
d_{i} \beta \in \tilde{R}_{n}
$$

for $i>0$. Consider the map of $\Delta$-groups $\tilde{\phi}: \tilde{R} \rightarrow \operatorname{Inn}(\hat{F})$. Then the elements

$$
\tilde{\phi}\left(d_{j} \beta\right) \in \operatorname{Inn}(\hat{F})_{n-1}, \quad 0<j \leq n,
$$


are matching faces with respect to 0. Recall from the proof of Proposition 6.3.6 that the morphism of $\Delta$-groups $F\left[S^{1}\right] \cong \hat{F} \rightarrow \operatorname{Inn}(\hat{F})$ induces an isomorphism

$$
F\left[S^{1}\right]_{j} \cong \operatorname{Inn}(\hat{F})_{j}
$$

for $j \geq 2$. Since $F\left[S^{1}\right]$ is a simplicial group, the $\Delta$-group $F\left[S^{1}\right]$ is fibrant. Thus there is an element $\alpha \in \operatorname{Inn}(\hat{F})_{n}$ such that

$$
d_{j} \alpha=\tilde{\phi}\left(d_{j} \beta\right)
$$

for $0<j \leq n$. By Lemma 6.3.9, the morphism of $\Delta$-groups $\tilde{\phi}: \tilde{R} \rightarrow \operatorname{Inn}(\hat{F})$ is an epimorphism. Therefore there is an element $\tilde{\alpha} \in(\tilde{R})_{n}=\tilde{R}_{n+1}$ such that $\tilde{\phi}(\tilde{\alpha})=\alpha$. Let $\tilde{\beta}=\beta \cdot \tilde{\alpha}^{-1} \in(\mathcal{P})_{n}$. Then $f_{*}(\tilde{\beta})=\bar{\beta}, \tilde{\phi}\left(d_{j} \tilde{\beta}\right)=1$ and $f_{*}\left(d_{j} \tilde{\beta}\right)=1$ for $j>0$. It follows that there exist integers $k_{j}$ such that $d_{j} \tilde{\beta}=\alpha_{n-1}^{k_{j}}$ for $0<j \leq n$, where, as in the proof of Proposition 6.3.6, $\alpha_{n-1}$ generates the center $\mathcal{Z}\left(P_{n}\right)$. By equation (14),

$$
k_{1}=k_{2}=\cdots=k_{n} \text {. }
$$

Let $\tilde{\beta}^{\prime}=\tilde{\beta} \cdot \alpha_{n}^{-k_{1}}$. Then $\tilde{\beta}^{\prime} \in N_{n} \mathcal{P}$ with $f_{*}\left(\tilde{\beta}^{\prime}\right)=\bar{\beta}$. This proves that

$$
N f_{*}: N \mathcal{P} \rightarrow N \mathcal{F}\left(S^{2}\right)^{\pi_{1}}
$$

is an epimorphism. Hence there is a short exact sequence of chains

$$
1 \longrightarrow N \tilde{R} \longrightarrow N \mathcal{P} \longrightarrow N \mathcal{F}\left(S^{2}\right)^{\pi_{1}} \longrightarrow 1 \text {. }
$$

For any Moore cycle $\beta \in \mathrm{Z}_{n} \mathcal{P}$, let $\tilde{\beta}$ be the braid in $\mathcal{P}_{n+1}$ obtained by adding the trivial string to the left of $\beta$; in other words, $d_{j} \tilde{\beta}=1$ for $j>0$ and $d_{0} \tilde{\beta}=\beta$. Thus $\beta \in \mathrm{B}_{n} \mathcal{P}$. Hence $\mathrm{B} \mathcal{P}=\mathrm{ZP}$, and so

$$
\begin{aligned}
\mathrm{B}_{n} \mathcal{F}\left(S^{2}\right)^{\pi_{1}} & =\operatorname{Im}\left(\mathrm{B}_{n} \mathcal{P} \rightarrow \mathcal{F}\left(S^{2}\right)_{n}^{\pi_{1}}\right) \\
& =\operatorname{Im}\left(\mathrm{Z}_{n} \mathcal{P} \rightarrow \mathcal{F}\left(S^{2}\right)_{n}^{\pi_{1}}\right) \\
& =\operatorname{Im}\left(\mathrm{Z}_{n} \mathcal{P} \rightarrow \mathrm{Z}_{n} \mathcal{F}\left(S^{2}\right)^{\pi_{1}}\right) \\
& =\operatorname{Im}\left(\operatorname{Brun}_{n+1}\left(D^{2}\right) \rightarrow \operatorname{Brun}_{n+1}\left(S^{2}\right)\right)
\end{aligned}
$$

for $n \geq 2$. By Theorem 1.1, there is an exact sequence

$$
1 \longrightarrow T_{n+1} \longrightarrow \operatorname{Brun}_{n+1}\left(D^{2}\right) \stackrel{f_{*}}{\longrightarrow} \operatorname{Brun}_{n+1}\left(S^{2}\right) \longrightarrow \pi_{n}\left(S^{2}\right) \longrightarrow 1
$$

for $n \geq 4$, where $T_{n+1}$ is the kernel of $f_{*}$. So $T_{n+1}=\mathrm{Z}_{n} \mathcal{P} \cap(\tilde{R})_{n}=\mathrm{Z}_{n} \tilde{R}$. By Proposition 6.3.11 and Theorem 6.2.3 there are isomorphisms

$$
\mathrm{Z}_{n} \tilde{R} \cong \mathrm{Z}_{n}\left(F\left[S^{1}\right]\right) \cong \operatorname{Brun}_{n+2}\left(S^{2}\right),
$$

completing the proof.

Note. From the proof, $\pi_{*}(\mathcal{P})=0$, and so $\mathcal{P}$ is a contractible simplicial group. Thus the simplicial group $\mathcal{P}$ can be regarded as a model for the total space of the classifying spaces of simplicial subgroups of $\mathcal{P}$. More generally, if $\mathcal{G}$ is a simplicial subgroup of $\mathcal{P}$, then the simplicial coset $\mathcal{P} / \mathcal{G}$ is homotopy equivalent to the classifying space of $\mathcal{G}$. 
6.5. Proof of Theorem 1.3. In higher dimensions, there is a version of Theorem 1.3 that follows from Theorem 1.2 by means of the following result.

Lemma 6.5.1. Suppose that for $n \geq k$ there is an exact sequence of groups

$$
1 \longrightarrow A_{n+1} \stackrel{\alpha_{n+1}}{\longrightarrow} B_{n} \stackrel{\beta_{n}}{\longrightarrow} A_{n} \stackrel{\gamma_{n}}{\longrightarrow} C_{n-1} \longrightarrow 1
$$

and a monomorphism $\alpha_{k}: A_{k} \rightarrow B_{k-1}$. For $n \geq k$, define $\partial_{n}=\alpha_{n} \beta_{n}: B_{n} \rightarrow B_{n-1}$. Then for $n \geq k$ we have $\partial_{n} \partial_{n+1}$ trivial, $\operatorname{Im}\left(\partial_{n+1}\right) \unlhd \operatorname{Ker}\left(\partial_{n}\right)$, and

$$
H_{n}\left(B_{*}, \partial\right) \cong C_{n} .
$$

Proof. The proof is largely straightforward. The only subtlety occurs in the assertion of normality, where the fact that for $n \geq k, \alpha_{n}$ is a monomorphism implies that $\operatorname{Ker}\left(\partial_{n}\right)=\operatorname{Ker}\left(\beta_{n}\right)=\alpha_{n+1}\left(A_{n+1}\right)$. Then

$$
\begin{aligned}
\operatorname{Im}\left(\partial_{n+1}\right) & =\alpha_{n+1}\left(\operatorname{Im}\left(\beta_{n+1}\right)\right) \\
& =\alpha_{n+1}\left(\operatorname{Ker}\left(\gamma_{n+1}\right)\right) \\
& \unlhd \alpha_{n+1}\left(A_{n+1}\right) .
\end{aligned}
$$

It follows that $H_{n}\left(B_{*}, \partial\right) \cong \alpha_{n+1}\left(A_{n+1} / \operatorname{Ker}\left(\gamma_{n+1}\right)\right) \cong \operatorname{Im}\left(\gamma_{n+1}\right)$.

In order to obtain the theorem in all dimensions, consider the following geometric construction of $\partial$. Let $\delta: F(\mathbb{C}, n+1) \longrightarrow F(\mathbb{C}, n)$ be the map defined by

$$
\delta\left(z_{0}, z_{1}, \ldots, z_{n}\right)=\left(\frac{1}{\bar{z}_{1}-\bar{z}_{0}}, \frac{1}{\bar{z}_{2}-\bar{z}_{0}}, \ldots, \frac{1}{\bar{z}_{n}-\bar{z}_{0}}\right),
$$

corresponding geometrically (coordinatewise) to inversion in $\mathbb{C}$ with respect to the unit circle centered at $z_{0}$. Consider the effect of $\delta$ on $B_{n+1}$ regarded as the subset (labelled $G_{n}$ in the proof of Theorem 3.2.12) of the fundamental groupoid consisting of path homotopy classes of paths starting at the basepoint. Choose the basepoint $\mathbf{q}_{n+1}=\left(q_{0}, q_{1}, \ldots, q_{n}\right)$ as follows. In order that the basepoint $\left(q_{0}, \ldots, q_{n}\right)$ behave well with respect to $\delta$, choose $q_{0}=0$ and let $q_{1}, \ldots, q_{n}$ be points, ordered clockwise, lying in the first quadrant of the unit circle. More precisely, as in Subsection 3.2. consider an embedding $\theta$ of $\mathbb{R}^{+}$in $D^{2} \subseteq \mathbb{C}$, say

$$
\theta: t \longmapsto \begin{cases}i t & 0 \leq t \leq 1, \\ \exp \left(\frac{i \pi}{2 t}\right) & t \geq 1,\end{cases}
$$

which induces an ordering $0=q_{0}<q_{1}<\cdots<q_{j}=\theta(j)<\cdots<q_{n}$. Since $q_{0}=0$ and for $i \geq 1, q_{i} \bar{q}_{i}=1$, we have

$$
\delta\left(q_{0}, q_{1}, \ldots, q_{n}\right)=\left(q_{1}, \ldots, q_{n}\right) .
$$

Choose the canonical path $\gamma$ in $F(\mathbb{C}, n)$ from

$$
\mathbf{q}_{n}=\left(q_{0}, q_{1}, \ldots, q_{n-1}\right)=(\theta(0), \theta(1), \ldots, \theta(n-1))
$$

to

$$
\left(q_{1}, q_{2}, \ldots, q_{n}\right)=(\theta(1), \theta(2), \ldots, \theta(n))
$$

given by

$$
u \mapsto(\theta(u), \theta(1+u), \ldots, \theta(n-1+u)), \quad 0 \leq u \leq 1 .
$$

This gives rise to an isomorphism of fundamental groups

$$
h_{[\gamma]}: \pi_{1}\left(F(\mathbb{C}, n),\left(q_{1}, q_{2}, \ldots, q_{n}\right)\right) \longrightarrow \pi_{1}\left(F(\mathbb{C}, n), \mathbf{q}_{n}\right) .
$$


There is a homomorphism

$$
\tilde{\partial}=h_{[\gamma]} \circ \delta_{*}: P_{n+1}=\pi_{1}\left(F(\mathbb{C}, n+1), \mathbf{q}_{n+1}\right) \longrightarrow P_{n}=\pi_{1}\left(F(\mathbb{C}, n), \mathbf{q}_{n}\right)
$$

with

$$
d_{i} \circ \tilde{\partial}=\tilde{\partial} \circ d_{i+1}
$$

for $0 \leq i \leq n-1$.

From the braid relations, there is an automorphism $\chi: B_{n} \longrightarrow B_{n}$ such that $\chi\left(\sigma_{i}\right)=\sigma_{i}^{-1}$ for all $i$. Since

$$
\chi\left(\sigma_{0} \sigma_{1} \cdots \sigma_{n-3} \sigma_{n-2}^{2} \sigma_{n-3} \cdots \sigma_{1} \sigma_{0}\right)=\left(\sigma_{0} \sigma_{1} \cdots \sigma_{n-3} \sigma_{n-2}^{2} \sigma_{n-3} \cdots \sigma_{1} \sigma_{0}\right)^{-1},
$$

by Lemma 6.3.8 the map $\chi: B_{n} \longrightarrow B_{n}$ induces an automorphism of $B_{n}\left(S^{2}\right)$. Because each $\sigma_{i}$ is an involution in the symmetric group, $\chi$ acts as the identity map on $S_{n}$, and so $\chi$ restricts to an automorphism on $P_{n}\left(S^{2}\right)$.

Let $\left\{A_{i, j} \mid 0 \leq i<j \leq n\right\}$ be the set of generators for $P_{n+1}$ defined in [6, Fig. 4 , p.21]; that is,

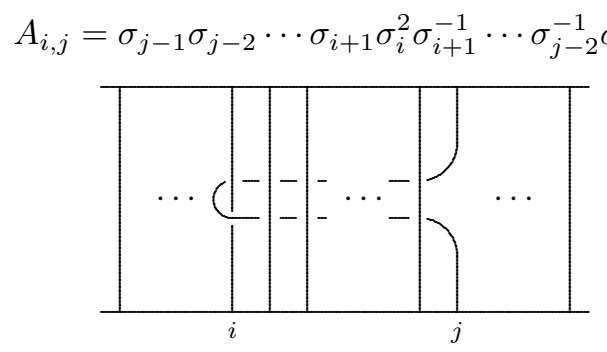

The generator $A_{i j}$ of $P_{n+1}$

To help with the computation of $\tilde{\partial}\left(A_{i, j}\right)$, it is convenient to introduce a new notation

$$
\begin{aligned}
A_{-1, j} & =\left(A_{j, j+1} A_{j, j+2} \cdots A_{j, n}\right)^{-1}\left(A_{0, j} A_{1, j} \cdots A_{j-1, j}\right)^{-1} \\
& =\left(\sigma_{j} \sigma_{j+1} \cdots \sigma_{n-2} \sigma_{n-1}^{2} \sigma_{n-2} \cdots \sigma_{j}\right)^{-1} \cdot\left(\sigma_{j-1} \cdots \sigma_{1} \sigma_{0}^{2} \sigma_{1} \cdots \sigma_{j-1}\right)^{-1}
\end{aligned}
$$

in $P_{n+1}$ for $0 \leq j \leq n$. This corresponds to the braid:

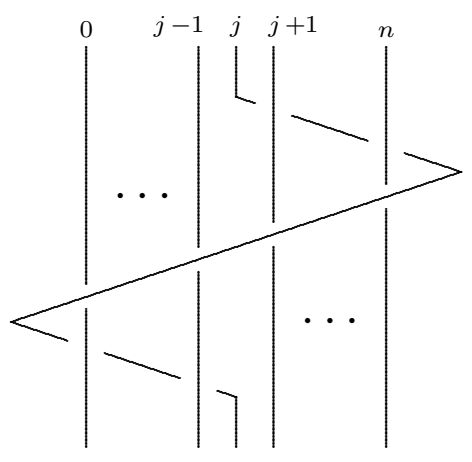

$A_{-1, j}$

Let $\partial=\chi \circ \tilde{\partial}: P_{n+1} \longrightarrow P_{n}$. 
Lemma 6.5.2. With the above notation, for each $n$ the group homomorphism $\partial: P_{n+1} \longrightarrow P_{n}$ satisfies:

(1) $\partial\left(A_{i, j}\right)=A_{i-1, j-1}$ whenever $0 \leq i<j$, and $\partial\left(A_{-1, j}\right)=1$;

(2) $d_{i} \circ \partial=\partial \circ d_{i+1}$ whenever $0 \leq i \leq n-1$;

(3) $\partial \circ \partial=\partial \circ d_{0}$.

Proof. As in the picture below, we have, for $0<i<j$,

$$
\partial\left(A_{i, j}\right)=\chi \circ \chi\left(A_{i-1, j-1}\right)=A_{i-1, j-1} .
$$
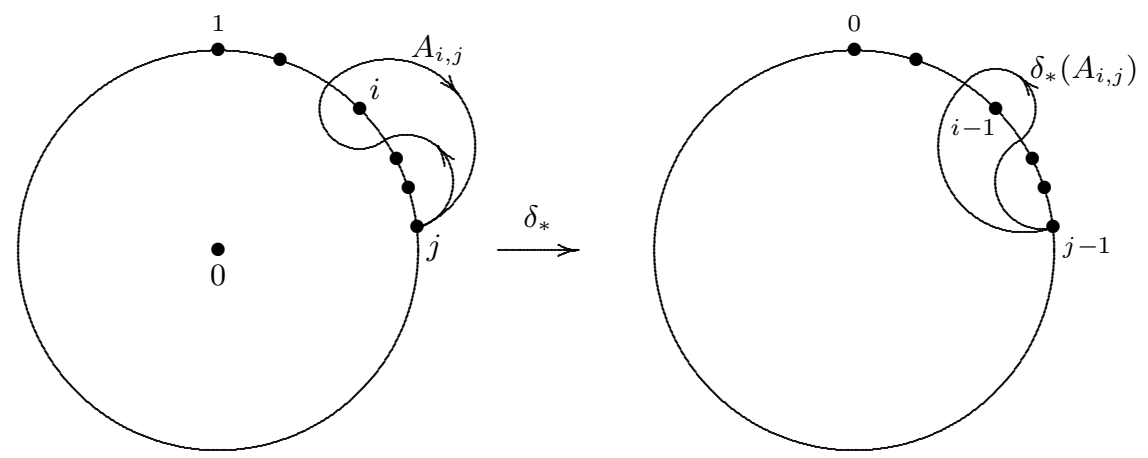

These pictures represent the view from above. Under $\delta$, the original 0-th strand is deleted, the labelling of the remainder of the configuration is shifted down by one, while the original $j$-th strand of $A_{i, j}$ is the only one to move, in the direction indicated by the arrow. For $i=0<j$, the situation looks as follows:
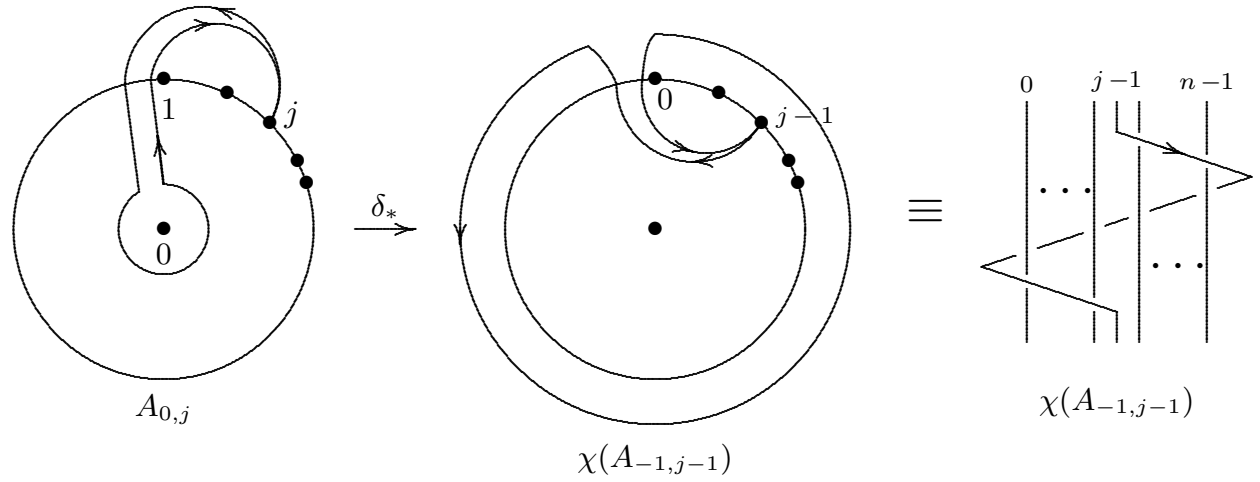

Thus

$$
\begin{aligned}
\partial\left(A_{0, j}\right) & =\chi\left(\sigma_{j-1} \cdots \sigma_{n-3} \sigma_{n-2}^{2} \sigma_{n-3} \cdots \sigma_{j-1}\right) \cdot \chi\left(\sigma_{j-2} \cdots \sigma_{1} \sigma_{0}^{2} \sigma_{1} \cdots \sigma_{j-2}\right) \\
& =\left(\sigma_{j-1} \cdots \sigma_{n-3} \sigma_{n-2}^{2} \sigma_{n-3} \cdots \sigma_{j-1}\right)^{-1} \cdot\left(\sigma_{j-2} \cdots \sigma_{1} \sigma_{0}^{2} \sigma_{1} \cdots \sigma_{j-2}\right)^{-1} \\
& =A_{-1, j-1} .
\end{aligned}
$$

These formulae imply that

$$
\begin{aligned}
\partial\left(A_{-1, j}\right)= & \left(A_{j-1, j} A_{j-1, j+1} \cdots A_{j-1, n-1}\right)^{-1} \\
& \cdot\left(A_{0, j-1} A_{1, j-1} \cdots A_{j-2, j-1}\right)^{-1} \cdot A_{-1, j-1}^{-1} \\
= & 1 .
\end{aligned}
$$


Hence assertion (1) follows. Note that

$$
d_{k}\left(A_{i, j}\right)= \begin{cases}A_{i-1, j-1} & k<i<j \\ 1 & k=i, j \\ A_{i, j-1} & i<k<j \\ A_{i, j} & k>j .\end{cases}
$$

Then assertions (2) and (3) follow from (1) by easy computation.

From formulae (2) and (3), the following is immediate.

Corollary 6.5.3. Let $\Gamma=\left\{\Gamma_{n}\right\}_{n \geq 0}$ be the sequence of groups defined by $\Gamma_{0}=1$ and, for $n \geq 1$,

$$
\Gamma_{n}=\mathcal{P}_{n-1}=P_{n}
$$

with faces $d_{0}(\Gamma)=\partial$ and $d_{k}(\Gamma)=d_{k-1}(\mathcal{P})$ for $k>0$. Then $\Gamma$ is a $\Delta$-group.

Proof of Theorem 1.3. From the fibre sequence

$$
\mathbb{R}^{2}-Q_{n} \longrightarrow F\left(\mathbb{R}^{2}, n+1\right) \stackrel{d_{n}}{\longrightarrow} F\left(\mathbb{R}^{2}, n\right),
$$

the kernel $G_{n}:=\operatorname{Ker}\left(d_{n}: P_{n+1} \longrightarrow P_{n}\right)$ is the free group of rank $n$ generated by

$$
A_{0, n}, A_{1, n}, \ldots, A_{n-1, n} .
$$

Observe that $d_{i}$ maps $G_{n}$ into $G_{n-1}$ for $0 \leq i \leq n-1$. By the above lemma, we have

$$
\partial\left(A_{i, n}\right)= \begin{cases}A_{i-1, n-1} & 0<i<n, \\ \left(A_{0, n-1} A_{1, n-1} \cdots A_{n-2, n-1}\right)^{-1} & i=0<n .\end{cases}
$$

Thus $\partial$ maps $G_{n}$ into $G_{n-1}$. Let $F\left[S^{1}\right]$ be the Milnor construction of $S^{1}$ with $F\left[S^{1}\right]_{n}=F\left(y_{0}, y_{1}, \ldots, y_{n-1}\right)$ and the faces described in Equation (11). Let

$$
\varphi: F\left[S^{1}\right]_{n} \longrightarrow G_{n}
$$

be the group isomorphism defined by

$$
\varphi\left(y_{i}\right)=A_{i, n}
$$

for $0 \leq i \leq n-1$. Then

$$
d_{i} \circ \varphi(w)=\varphi \circ d_{i+1}(w), \quad \partial \circ \varphi(w)=\varphi\left(d_{0}(w)\right)
$$

for $w \in F\left[S^{1}\right]_{n}$. Thus the map $\varphi$ induces an isomorphism

$$
N_{n} F\left[S^{1}\right] \stackrel{\cong}{\longrightarrow} G_{n} \cap \bigcap_{i=0}^{n-1} \operatorname{Ker}\left(d_{i}: P_{n+1} \rightarrow P_{n}\right)=\operatorname{Brun}_{n+1}\left(D^{2}\right)
$$

with $\partial \circ \varphi(w)=\varphi\left(d_{0}(w)\right)$ for $w \in N_{n} F\left[S^{1}\right]$. Hence

$$
\partial \circ \partial: \operatorname{Brun}_{n+1}\left(D^{2}\right) \longrightarrow \operatorname{Brun}_{n-1}\left(D^{2}\right)
$$

is trivial and

$$
H_{n+1}\left(\operatorname{Brun}\left(D^{2}\right), \partial\right) \cong H_{n}\left(N F\left[S^{1}\right], d_{0}\right) \cong \pi_{n}\left(\Omega S^{2}\right) \cong \pi_{n+1}\left(S^{2}\right)
$$

for all $n$. This finishes the proof.

Observe that we have the Moore chains $N \Gamma_{n}=\operatorname{Brun}_{n}\left(D^{2}\right)$ with $d_{0}(\Gamma)=\partial$. So the proof gives the following. 
Corollary 6.5.4. Let $\Gamma$ be the $\Delta$-group defined in Corollary 6.5.3, Then

$$
\pi_{n}(\Gamma) \cong \pi_{n}\left(S^{2}\right)
$$

for all $n$.

6.6. Comparison of differentials. In this subsection, we investigate the relation between the differentials in Lemmas 6.5.1 and 6.5.2. This enables us to deduce Theorem 1.2 from Theorem 1.3 . The group homomorphism

$$
\chi: B_{n} \longrightarrow B_{n}
$$

is in fact induced by complex conjugation. The map

$$
J: F(\mathbb{C}, n) \longrightarrow F(\mathbb{C}, n) \quad\left(z_{0}, \ldots, z_{n-1}\right) \mapsto\left(\bar{z}_{0}, \ldots, \bar{z}_{n-1}\right)
$$

induces a map $\bar{J}: B(\mathbb{C}, n)=F(\mathbb{C}, n) / S_{n} \longrightarrow B(\mathbb{C}, n)$ and so a group homomorphism $\bar{J}_{*}: B_{n}=\pi_{1}(B(\mathbb{C}, n)) \longrightarrow B_{n}$. By choosing the basepoint with real coordinates, one can easily show the following proposition, where the generators $\sigma_{i}$ of $B_{n}$ are given in Birman's sense [6].

Proposition 6.6.1. With the above notation, $\bar{J}_{*}=\chi: B_{n} \longrightarrow B_{n}$.

Recall that the group $\hat{F}_{n}$ is generated by $\hat{z}_{0}, \ldots, \hat{z}_{n-1}$ with the single relation $\hat{z}_{0} \hat{z}_{1} \cdots \hat{z}_{n-1}=1$. Let $\phi: B_{n} \longrightarrow \operatorname{Aut}\left(\hat{F}_{n}\right)$ denote the reduced Artin representation. Recall from Lemma 6.3.8 that

$$
\nu_{n}=\sigma_{0} \sigma_{1} \cdots \sigma_{n-2} \sigma_{n-1}^{2} \sigma_{n-2} \cdots \sigma_{1} \sigma_{0}=A_{-1,0}^{-1}
$$

is the normal generator for the kernel of $f_{*}: P_{n+1} \longrightarrow P_{n+1}\left(S^{2}\right)$.

Lemma 6.6.2. Let $A_{i, j}$ be the generators for $P_{n+1}$ given in [6]. Then, for $j \geq 1$, as automorphisms of $\hat{F}_{n}$,

$$
\left(\phi \circ \partial\left(A_{0, j}\right)\right)(x)=\hat{z}_{j-1} x \hat{z}_{j-1}^{-1}
$$

and

$$
\phi \circ \partial\left(A_{0,1} A_{0,2} \cdots A_{0, n}\right)=\operatorname{id} \in \operatorname{Aut}\left(\hat{F}_{n}\right) .
$$

Proof. Writing $\phi(\beta)(z)=\beta \cdot z$, we calculate that

$$
\begin{aligned}
\nu_{n-1} \cdot \hat{z}_{k}= & \sigma_{0} \sigma_{1} \cdots \sigma_{n-3} \sigma_{n-2}^{2} \sigma_{n-3} \cdots \sigma_{1} \sigma_{0} \cdot \hat{z}_{k} \\
= & \sigma_{0} \sigma_{1} \cdots \sigma_{n-3} \sigma_{n-2}^{2} \sigma_{n-3} \cdots \sigma_{k} \sigma_{k-1} \cdot \hat{z}_{k} \\
= & \sigma_{0} \sigma_{1} \cdots \sigma_{n-3} \sigma_{n-2}^{2} \sigma_{n-3} \cdots \sigma_{k} \cdot \hat{z}_{k}^{-1} \hat{z}_{k-1} \hat{z}_{k} \\
= & \sigma_{0} \sigma_{1} \cdots \sigma_{n-3} \sigma_{n-2} \cdot \hat{z}_{n-1}^{-1} \hat{z}_{k-1} \hat{z}_{n-1} \\
= & \sigma_{0} \sigma_{1} \cdots \sigma_{k-1} \cdot\left(\hat{z}_{n-1}^{-1} \hat{z}_{n-2}^{-1} \cdots \hat{z}_{k+1}^{-1} \hat{z}_{k}^{-1} \hat{z}_{k+1} \cdots \hat{z}_{n-1}\right) \hat{z}_{k-1} \\
& \left(\hat{z}_{n-1}^{-1} \hat{z}_{n-2}^{-1} \cdots \hat{z}_{k+1}^{-1} \hat{z}_{k} \hat{z}_{k+1} \cdots \hat{z}_{n-1}\right) \\
= & \sigma_{0} \sigma_{1} \cdots \sigma_{k-2} \cdot\left(\hat{z}_{n-1}^{-1} \hat{z}_{n-2}^{-1} \cdots \hat{z}_{k}^{-1} \hat{z}_{k-1}^{-1} \hat{z}_{k} \cdots \hat{z}_{n-1}\right) \hat{z}_{k} \\
& \left(\hat{z}_{n-1}^{-1} \hat{z}_{n-2}^{-1} \cdots \hat{z}_{k}^{-1} \hat{z}_{k-1} \hat{z}_{k} \cdots \hat{z}_{n-1}\right) \\
= & \left(\hat{z}_{n-1}^{-1} \hat{z}_{n-2}^{-1} \cdots \hat{z}_{1}^{-1} \hat{z}_{0}^{-1} \hat{z}_{1} \cdots \hat{z}_{n-2} \hat{z}_{n-1}\right) \hat{z}_{k}\left(\hat{z}_{n-1}^{-1} \hat{z}_{n-2}^{-1} \cdots \hat{z}_{1}^{-1} \hat{z}_{0} \hat{z}_{1} \cdots \hat{z}_{n-1}\right) \\
= & \hat{z}_{0}^{-1} \hat{z}_{k} \hat{z}_{0} .
\end{aligned}
$$

Thus, for all words $x \in \hat{F}_{n}$, the element $\nu_{n-1} \cdot x=\hat{z}_{0}^{-1} x \hat{z}_{0}$, and so

$$
\nu_{n-1}^{-1} \cdot x=\hat{z}_{0} x \hat{z}_{0}^{-1} \text {. }
$$


Therefore for any braid $\alpha$, the action

$$
\alpha \nu_{n-1}^{-1} \alpha^{-1} \cdot x=\alpha\left(\hat{z}_{0}\right) x \alpha\left(\hat{z}_{0}\right)^{-1} .
$$

Observe from the definition that

$$
A_{-1, j-1}=\left(\sigma_{j-2} \sigma_{j-3} \cdots \sigma_{0}\right) \nu_{n-1}^{-1}\left(\sigma_{j-2} \sigma_{j-3} \cdots \sigma_{0}\right)^{-1} .
$$

Since

$$
\sigma_{j-2} \sigma_{j-3} \cdots \sigma_{0}\left(\hat{z}_{0}\right)=\hat{z}_{j-1}
$$

the result follows.

Lemma 6.6.3. There is a group homomorphism $\alpha: P_{n+1}\left(S^{2}\right) \longrightarrow P_{n}$ such that the following diagram commutes:

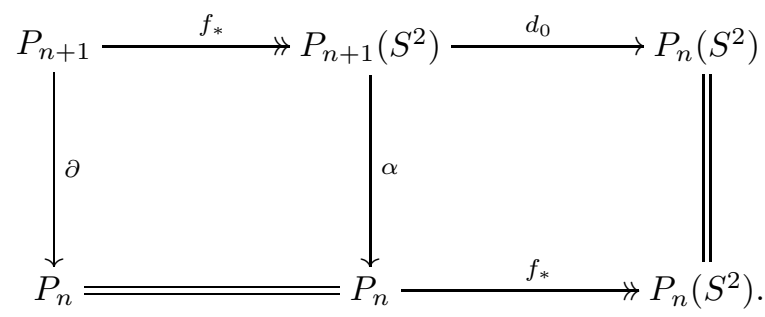

Proof. From Equation 15, each $\partial\left(A_{0, j}\right)$ goes to 1 in $P_{n}\left(S^{2}\right)$; also $\partial\left(A_{i, j}\right)=A_{i-1, j-1}$ for $i>0$. Thus

$$
f_{*} \circ \partial=d_{0} \circ f_{*}=f_{*} \circ d_{0} .
$$

Since $\partial\left(\nu_{n}\right)=\partial\left(A_{-1,0}^{-1}\right)=1$ and $\nu_{n}$ normally generates $\operatorname{Ker}\left(f_{*}\right)$, the homomorphism $\partial$ factors through $f_{*}: P_{n+1} \longrightarrow P_{n+1}\left(S^{2}\right)$. Let $\alpha: P_{n+1}\left(S^{2}\right) \longrightarrow P_{n}$ be the resulting homomorphism. Then $f_{*} \circ \alpha=d_{0}$ because $f_{*}: P_{n+1} \longrightarrow P_{n+1}\left(S^{2}\right)$ is an epimorphism.

From the fibre sequence

$$
S^{2} \backslash Q_{n} \stackrel{j}{\longrightarrow} F\left(S^{2}, n+1\right) \stackrel{d_{0}}{\longrightarrow} F\left(S^{2}, n\right),
$$

we have the exact sequence

$\pi_{2}\left(F\left(S^{2}, n\right)\right) \longrightarrow \hat{F}_{n}=\pi_{1}\left(S^{2} \backslash Q_{n}\right) \stackrel{j_{*}}{\longrightarrow} P_{n+1}\left(S^{2}\right)=\pi_{1}\left(F\left(S^{2}, n+1\right)\right) \stackrel{d_{0}}{\longrightarrow} P_{n}\left(S^{2}\right)$.

By Lemma 6.6.3, there is a commutative diagram whose rows are exact sequences:

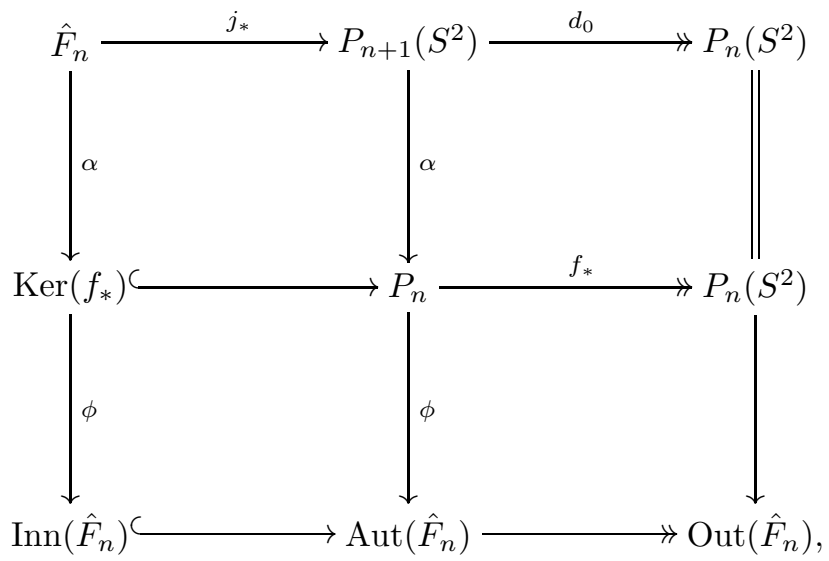


where the lower two rows are from the Artin representation of $P_{n}$ and Lemma 6.3.9.

Lemma 6.6.4. For $n \geq 3$, the composite

$$
\hat{F}_{n} \stackrel{\alpha}{\longrightarrow} \operatorname{Ker}\left(f_{*}\right) \stackrel{\phi}{\longrightarrow} \operatorname{Inn}\left(\hat{F}_{n}\right)
$$

is an isomorphism.

Proof. Recall that $\hat{F}_{n}=\pi_{1}\left(S^{2} \backslash Q_{n}\right)$ is generated by $A_{0,1}, A_{0,2}, \ldots, A_{0, n}$ with the single relation $A_{0,1} A_{0,2} \cdots A_{0, n}=1$. By Lemma 6.6.2 $\phi \circ \alpha\left(A_{0, j}\right)$ is the conjugation induced by the word $\hat{z}_{j-1}$ in $\hat{F}_{n}$. The assertion follows from the fact that

$$
\operatorname{Inn}\left(\hat{F}_{n}\right) \cong \hat{F}_{n}
$$

for $n \geq 3$.

With the aid of these results, we can now recapture Theorem 1.2 from Theorem 1.3.

Theorem 6.6.5. For $n \geq 5$, the sequence

$$
1 \longrightarrow \operatorname{Brun}_{n+1}\left(S^{2}\right) \stackrel{\alpha}{\longrightarrow} \operatorname{Brun}_{n}\left(D^{2}\right) \stackrel{f_{*}}{\longrightarrow} \operatorname{Brun}_{n}\left(S^{2}\right) \longrightarrow \pi_{n-1}\left(S^{2}\right) \longrightarrow 1
$$

is exact, and the algebraic differential $\alpha f_{*}$ coincides with the geometric differential $\partial$.

Proof. The comparison of differentials occurs in Lemma 6.6.3. To obtain the exact sequence (that is, Theorem 1.2), observe first that $f_{*}: \mathcal{P} \longrightarrow \mathcal{F}\left(S^{2}\right)^{\pi_{1}}$ is an epimorphism of $\Delta$-groups. Thus $f_{*}\left(\operatorname{Brun}_{n}\left(D^{2}\right)\right) \unlhd P_{n}\left(S^{2}\right)$, and so for $n \geq 3$ we may define

$$
C_{n-1}=\operatorname{Brun}_{n}\left(S^{2}\right) / f_{*}\left(\operatorname{Brun}_{n}\left(D^{2}\right)\right)
$$

By Lemmas 6.5.2 and 6.6.3, for $\alpha: P_{n+1}\left(S^{2}\right) \longrightarrow P_{n}$, the formula

$$
d_{i} \circ \alpha=\alpha \circ d_{i+1}
$$

holds for $0 \leq i \leq n-1$. Thus $\alpha$ maps $\operatorname{Brun}_{n+1}\left(S^{2}\right)$ into $\operatorname{Brun}_{n}\left(D^{2}\right) \cap \operatorname{Ker}\left(f_{*}\right)$. Note that the homomorphism $j_{*}: \hat{F}_{n}=\pi_{1}\left(S^{2} \backslash Q_{n}\right) \longrightarrow P_{n+1}\left(S^{2}\right)$ satisfies

$$
d_{i} \circ j_{*}=j_{*} \circ d_{i+1}
$$

for $0 \leq i \leq n-1$. Thus the composite $\hat{F}_{n} \stackrel{\alpha}{\longrightarrow} \operatorname{Ker}\left(f_{*}\right) \stackrel{\phi}{\longrightarrow} \operatorname{Inn}\left(\hat{F}_{n}\right)$ induces a morphism of $\Delta$-groups

$$
\phi \circ \alpha: \hat{F}=\left\{\hat{F}_{n+1}\right\}_{n \geq 0} \longrightarrow \operatorname{Inn}(\hat{F})=\left\{\operatorname{Inn}\left(\hat{F}_{n+1}\right)\right\}_{n \geq 0}
$$

with $\phi \circ \alpha: \hat{F}_{n} \longrightarrow \operatorname{Inn}\left(\hat{F}_{n}\right)$ an isomorphism by Lemma 6.6.4. It follows that

$$
\phi \circ \alpha: \mathrm{Z}_{n} \hat{F} \rightarrow \mathrm{Z}_{n} \operatorname{Inn}(\hat{F})
$$

is an isomorphism for $n \geq 3$. By the proof of Proposition 6.3.6, the reduced Artin representation $\phi$ induces an isomorphism

$$
\phi: \operatorname{Ker}\left(f_{*}\right) \cap \operatorname{Brun}_{n+1}\left(D^{2}\right) \longrightarrow \mathrm{Z}_{n} \operatorname{Inn}(\hat{F})
$$

for $n \geq 3$. Thus $\alpha: \operatorname{Brun}_{4}\left(S^{2}\right) \longrightarrow \operatorname{Brun}_{3}\left(D^{2}\right)$ is a monomorphism, and for $n \geq 4$,

$$
\alpha: \operatorname{Brun}_{n+1}\left(S^{2}\right) \longrightarrow \operatorname{Brun}_{n}\left(D^{2}\right) \cap \operatorname{Ker}\left(f_{*}\right)
$$

is an isomorphism and there is an exact sequence

$$
1 \longrightarrow \operatorname{Brun}_{n+1}\left(S^{2}\right) \stackrel{\alpha}{\longrightarrow} \operatorname{Brun}_{n}\left(D^{2}\right) \stackrel{f_{*}}{\longrightarrow} \operatorname{Brun}_{n}\left(S^{2}\right) \longrightarrow C_{n-1} \longrightarrow 1 .
$$

The result now follows by combining Lemma 6.5.1 with Theorem 1.3 . 


\section{LOW-DIMENSIONAL BRUNNIAN BRAIDS}

Explicit computations in low-dimensional cases are given in this subsection.

7.1. The Moore homotopy groups $\pi_{n}\left(\mathcal{F}\left(S^{2}\right)^{\pi_{1}}\right)$ for $n \leq 3$. The terminology in Subsection 6.2 is used here. Let $\mathcal{E}=\left\{F\left(S^{2}, n+1\right)\right\}_{n \geq 0}$. Recall that $\mathcal{E}^{\pi_{1}}=\mathcal{F}\left(S^{2}\right)^{\pi_{1}}$. Clearly

and

$$
\pi_{0}\left(\mathcal{E}^{\pi_{1}}\right)=\pi_{1}\left(F\left(S^{2}, 1\right)\right)=\pi_{1}\left(S^{2}\right)=1
$$

$$
\pi_{1}\left(\mathcal{E}^{\pi_{1}}\right) \subseteq \pi_{1}\left(F\left(S^{2}, 2\right)\right)=\pi_{1}\left(S^{2}\right)=1 .
$$

1. $\pi_{2}\left(\mathcal{F}\left(S^{2}\right)^{\pi_{1}}\right)$. Observe from Lemma 6.2.1 that

$$
\Omega\left(\mathcal{E}^{\pi_{1}}\right)_{2} \cong F\left[S^{1}\right]_{2}=\hat{F}\left(\hat{z}_{0}, \hat{z}_{1}, \hat{z}_{2}\right) .
$$

From Proposition 4.2.4, there is a short exact sequence

$$
0 \longrightarrow \mathbb{Z} \longrightarrow \pi_{1}\left((\tilde{\Omega} \mathcal{E})_{1}\right) \longrightarrow \mathbb{Z} / 2=\pi_{1}\left(F\left(S^{2}, 3\right)\right) \longrightarrow 1
$$

Thus there is a commutative diagram

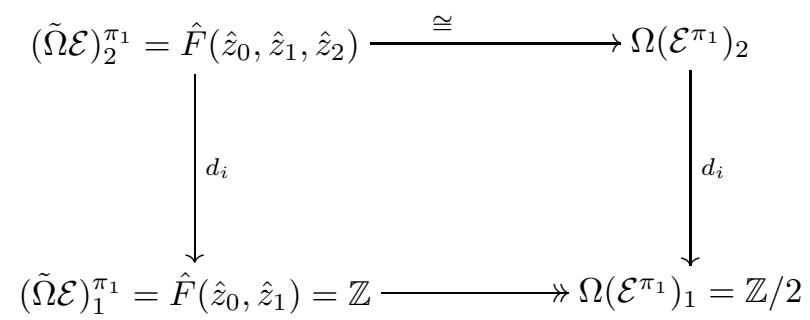

for $0 \leq i \leq 2$. Let $\alpha$ be nontrivial in $\mathbb{Z} / 2$. From Equation 12,

$$
d_{i}: \Omega\left(\mathcal{E}^{\pi_{1}}\right)_{2}=\hat{F}\left(\hat{z}_{0}, \hat{z}_{1}, \hat{z}_{2}\right) \cong F\left(\hat{z}_{0}, \hat{z}_{1}\right) \rightarrow \mathbb{Z} / 2
$$

is given by

$$
d_{0}\left(\hat{z}_{0}\right)=1, d_{0}\left(\hat{z}_{1}\right)=\alpha, d_{1}\left(\hat{z}_{0}\right)=\alpha, d_{1}\left(\hat{z}_{1}\right)=1, d_{2}\left(\hat{z}_{0}\right)=\alpha \text { and } d_{2}\left(\hat{z}_{1}\right)=\alpha .
$$

Let $w=\hat{z}_{0}^{l_{1}} \hat{z}_{1}^{k_{1}} \cdots \hat{z}_{0}^{l_{t}} \hat{z}_{1}^{k_{t}}$ be a word in $F\left(\hat{z}_{0}, \hat{z}_{1}\right)$. Then

(1) $w \in \operatorname{Ker}\left(d_{1}\right)$ if and only if $l_{1}+l_{2}+\cdots+l_{t} \equiv 0 \bmod 2$, and

(2) $w \in \operatorname{Ker}\left(d_{1}\right) \cap \operatorname{Ker}\left(d_{2}\right)=N \Omega\left(\mathcal{E}^{\pi_{1}}\right)_{2}$ if and only if

$$
l_{1}+l_{2}+\cdots+l_{t} \equiv k_{1}+k_{2}+\cdots+k_{t} \equiv 0 \bmod 2 .
$$

It follows that

$$
\mathrm{Z}_{2} \Omega\left(\mathcal{E}^{\pi_{1}}\right)=N_{2} \Omega\left(\mathcal{E}^{\pi_{1}}\right)
$$

Thus $\mathrm{B}_{1} \Omega\left(\mathcal{E}^{\pi_{1}}\right)=d_{0} N_{2} \Omega\left(\mathcal{E}^{\pi_{1}}\right)=1$ and so $\pi_{1}\left(\Omega\left(\mathcal{E}^{\pi_{1}}\right)\right)=\Omega\left(\mathcal{E}^{\pi_{1}}\right)_{1}$. Hence

$$
\pi_{2}\left(\mathcal{E}^{\pi_{1}}\right)=\pi_{1}\left(\Omega\left(\mathcal{E}^{\pi_{1}}\right)\right)=\Omega\left(\mathcal{E}^{\pi_{1}}\right)_{1}=\mathbb{Z} / 2 .
$$

2. $\pi_{3}\left(\mathcal{F}\left(S^{2}\right)^{\pi_{1}}\right)$. By Lemma 6.2.1 and Proposition 4.2.4, for $n \geq 2$ there are isomorphisms

$$
F\left[S^{1}\right]_{n} \cong \Omega\left(\mathcal{E}^{\pi_{1}}\right)_{n} \quad \text { and } \quad N_{3} F\left[S^{1}\right] \cong N_{3} \Omega\left(\mathcal{E}^{\pi_{1}}\right) .
$$

Thus

$$
\mathrm{B}_{2} F\left[S^{1}\right] \cong \mathrm{B}_{2} \Omega\left(\mathcal{E}^{\pi_{1}}\right)
$$

According to [70, Example 2.23 and Corollary 4.7],

$$
\mathrm{B}_{2} F\left[S^{1}\right]=\Gamma_{3} F\left(\hat{z}_{0}, \hat{z}_{1}\right)
$$


where $\Gamma_{3} G=[G,[G, G]]$. Thus $\pi_{3}\left(\mathcal{E}^{\pi_{1}}\right)=\pi_{2}\left(\Omega\left(\mathcal{E}^{\pi_{1}}\right)\right)$ is the kernel of the canonical map $F\left(\hat{z}_{0}, \hat{z}_{1}\right) / \Gamma_{3} \rightarrow \mathbb{Z} / 2 \times \mathbb{Z} / 2$, with the commutative diagram

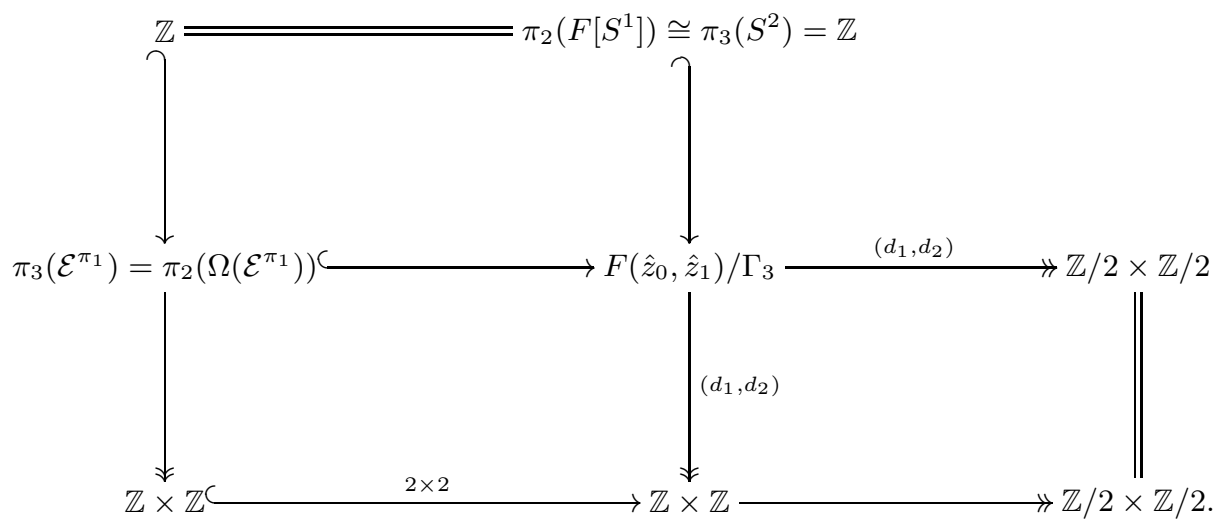

Thus there is a central extension

$$
0 \longrightarrow \mathbb{Z} \longrightarrow \pi_{3}\left(\mathcal{E}^{\pi_{1}}\right) \longrightarrow \mathbb{Z} \times \mathbb{Z} \longrightarrow 0
$$

Let $w_{0}=\hat{z}_{0}^{2}, w_{1}=\hat{z}_{1}^{2}$ and $\delta=\left[\hat{z}_{0}, \hat{z}_{1}\right]$ be elements in the integral Heisenberg group $F\left(\hat{z}_{0}, \hat{z}_{1}\right) / \Gamma_{3}$. Then $\pi_{3}\left(\mathcal{E}^{\pi_{1}}\right)$ is generated by $w_{0}, w_{1}$ and $\delta$ subject to the relations $\left[w_{0}, \delta\right]=\left[w_{1}, \delta\right]=1$ and $\left[w_{0}, w_{1}\right]=\delta^{4}$. In other words, it has a faithful representation as the matrix group generated by

$$
\left[\begin{array}{lll}
1 & 2 & 0 \\
0 & 1 & 0 \\
0 & 0 & 1
\end{array}\right],\left[\begin{array}{lll}
1 & 0 & 0 \\
0 & 1 & 2 \\
0 & 0 & 1
\end{array}\right],\left[\begin{array}{lll}
1 & 0 & 1 \\
0 & 1 & 0 \\
0 & 0 & 1
\end{array}\right] .
$$

In summary, the following is proved:

Proposition 7.1.1. The group $\pi_{3}\left(\mathcal{F}\left(S^{2}\right)^{\pi_{1}}\right)$ is generated by $w_{0}, w_{1}$ and $\delta$ subject to the relations $\left[w_{0}, \delta\right]=\left[w_{1}, \delta\right]=1$ and $\left[w_{0}, w_{1}\right]=\delta^{4}$, with a linear representation given above, and with center isomorphic to $\pi_{3}\left(S^{2}\right)$ as above.

7.2. The Brunnian groups $\operatorname{Brun}_{n}\left(S^{2}\right)$ for $n \leq 4$. Clearly

$$
\operatorname{Brun}_{1}\left(S^{2}\right)=1 \quad \text { and } \operatorname{Brun}_{2}\left(S^{2}\right)=\pi_{1}\left(B\left(S^{2}, 2\right)\right)=\mathbb{Z} / 2 .
$$

By Proposition 4.2.2, $\operatorname{Brun}_{n}\left(S^{2}\right) \subseteq \pi_{1}\left(F\left(S^{2}, n\right)\right)$ for $n \geq 3$. Thus

$$
\operatorname{Brun}_{3}\left(S^{2}\right)=\pi_{1}\left(F\left(S^{2}, 3\right)\right)=\mathbb{Z} / 2 .
$$

To determine $\operatorname{Brun}_{4}\left(S^{2}\right)$, first observe that

$$
\operatorname{Brun}_{4}\left(S^{2}\right)=\mathrm{Z}_{3} \mathcal{E}^{\pi_{1}}=\mathrm{Z}_{2} \Omega\left(\mathcal{E}^{\pi_{1}}\right)=N_{2} \Omega\left(\mathcal{E}^{\pi_{1}}\right),
$$

with the last equality from Equation (16). Now from Lemma 6.2.1 and Equation (16), there is a short exact sequence

$$
1 \longrightarrow \operatorname{Brun}_{4}\left(S^{2}\right)=N_{2} \Omega\left(\mathcal{E}^{\pi_{1}}\right) \longrightarrow F\left(\hat{z}_{0}, \hat{z}_{1}\right) \stackrel{\left(d_{1}, d_{2}\right)}{\longrightarrow} \mathbb{Z} / 2 \times \mathbb{Z} / 2 \longrightarrow 1 .
$$

Let $H$ be the subgroup of $F\left(\hat{z}_{0}, \hat{z}_{1}\right)$ generated by the elements

$$
T=\left\{\hat{z}_{0}^{2}, \hat{z}_{1}^{2},\left[\hat{z}_{0}, \hat{z}_{1}\right], \hat{z}_{1}^{-1} \hat{z}_{0}^{2} \hat{z}_{1}, \hat{z}_{0}^{-1} \hat{z}_{1}^{2} \hat{z}_{0}\right\}
$$


Then $H$ is a subgroup of $\operatorname{Brun}_{4}\left(S^{2}\right)$, and from the short exact sequence it is clear that $H$ normally generates $\operatorname{Brun}_{4}\left(S^{2}\right)$. Write $a^{b}$ for $b^{-1} a b$. From the equations 50 , Theorem 5.1]

$$
\begin{gathered}
{\left[\hat{z}_{0}^{2}, \hat{z}_{1}\right]=\left[\hat{z}_{0}, \hat{z}_{1}\right]\left[\left[\hat{z}_{0}, \hat{z}_{1}\right], \hat{z}_{0}\right]\left[\hat{z}_{0}, \hat{z}_{1}\right] \text { and }} \\
\left(\left(\hat{z}_{0}^{2}\right)^{\hat{z}_{1}}\right)^{\hat{z}_{0}}=\left(\hat{z}_{0}^{2}\right)^{\hat{z}_{1} \hat{z}_{0}}=\left[\hat{z}_{0}, \hat{z}_{1}\right] \hat{z}_{1}^{-1} \hat{z}_{0}^{2} \hat{z}_{1}\left[\hat{z}_{0}, \hat{z}_{1}\right]^{-1},
\end{gathered}
$$

it follows that $H^{\hat{z}_{0}} \subseteq H$. Similarly, $H^{\hat{z}_{1}} \subseteq H$. Thus $H$ is a normal subgroup of $F\left(\hat{z}_{0}, \hat{z}_{1}\right)$ and so $H=\operatorname{Brun}_{4}\left(S^{2}\right)$. In other words, $\operatorname{Brun}_{4}\left(S^{2}\right)$ is the subgroup of $F\left(\hat{z}_{0}, \hat{z}_{1}\right)$ generated by $T$.

This proves the following:

Proposition 7.2.1. The Brunnian group $\operatorname{Brun}_{4}\left(S^{2}\right)$ is isomorphic to the subgroup of the free group $F\left(\hat{z}_{0}, \hat{z}_{1}\right)$ generated by the elements

$$
T=\left\{\hat{z}_{0}^{2}, \hat{z}_{1}^{2},\left[\hat{z}_{0}, \hat{z}_{1}\right], \hat{z}_{1}^{-1} \hat{z}_{0}^{2} \hat{z}_{1}, \hat{z}_{0}^{-1} \hat{z}_{1}^{2} \hat{z}_{0}\right\},
$$

in other words, the second term of the mod 2 descending central series for the group $F\left(\hat{z}_{0}, \hat{z}_{1}\right)$.

In geometry, recall from Theorem 2.2 that there is a homeomorphism

$$
\left(\mathbb{C P}^{1} \backslash\{0,1, \infty\}\right) \times P \mathrm{GL}_{2}(\mathbb{C}) \approx \mathrm{GP}\left(\mathbb{C P}^{1}, 4\right)=F\left(\mathbb{C P}^{1}, 4\right) .
$$

By taking the universal covering $U(X) \rightarrow X$, there is a decomposition

$$
U\left(\mathbb{C P}^{1} \backslash\{0,1, \infty\}\right) \times U P \mathrm{GL}_{2}(\mathbb{C}) \approx U F\left(\mathbb{C P}^{1}, 4\right) .
$$

(Note that $U P \mathrm{FL}_{2}(\mathbb{C}) \simeq S^{3}$ and $\mathbb{C P}^{1} \backslash\{0,1, \infty\} \simeq S^{1} \vee S^{1}$.) Observe that the map $\mathbb{C P}^{1} \backslash\{0,1, \infty\} \longrightarrow F\left(\mathbb{C P}^{1}, 4\right)$ induces the group homomorphism

$$
F\left(\hat{z}_{0}, \hat{z}_{1}\right) \cong \hat{F}\left(\hat{z}_{0}, \hat{z}_{1}, \hat{z}_{2}\right)=\pi_{1}\left(\mathbb{C P}^{1} \backslash\{0,1, \infty\}\right) \longrightarrow \pi_{1}\left(F\left(\mathbb{C P}^{1}, 4\right)\right)
$$

on fundamental groups. Since $\operatorname{Brun}_{4}\left(S^{2}\right) \subseteq F\left(\hat{z}_{0}, \hat{z}_{1}\right)$, the group $\operatorname{Brun}_{4}\left(S^{2}\right)$ acts on $U\left(\mathbb{C P}^{1} \backslash\{0,1, \infty\}\right)$ and the resulting covering

$$
E_{4}=\left(\operatorname{Brun}_{4}\left(S^{2}\right) \backslash U\left(\mathbb{C P}^{1} \backslash\{0,1, \infty\}\right)\right) \times U P \mathrm{GL}_{2}(\mathbb{C}) \longrightarrow F\left(\mathbb{C P}^{1}, 4\right)
$$

is a principal $\mathbb{Z} / 2 \times \mathbb{Z} / 2 \times \mathbb{Z} / 2$-bundle over $F\left(\mathbb{C P}^{1}, 4\right)$. The space $E_{4}$ is a manifold whose fundamental group is $\operatorname{Brun}_{4}\left(S^{2}\right)$.

The generators for $\operatorname{Brun}_{4}\left(S^{2}\right)$ in terms of braids are described below. Consider the short exact sequence of groups

$$
1 \longrightarrow \pi_{1}\left(S^{2}-Q_{3}\right) \longrightarrow \pi_{1}\left(F\left(S^{2}, 4\right)\right) \stackrel{d_{0}}{\longrightarrow} \pi_{1}\left(F\left(S^{2}, 3\right)\right),
$$

where $Q_{3}=\left\{q_{1}, q_{2}, q_{3}\right\}$. Let $q_{0}$ be a base point of $D^{2}-Q_{2}$. We can identify a word

$$
w \in F\left(\hat{z}_{0}, \hat{z}_{1}\right)=\pi_{1}\left(D^{2}-Q_{2}\right) \cong \pi_{1}\left(S^{2}-Q_{3}\right)
$$

with a pure braid in the following way.

Let $\lambda$ be a loop in $D^{2}-Q_{2}$ such that the homotopy class $[\lambda]=w$. Then $\lambda$ defines a string $\lambda_{0}: I \rightarrow\left(D^{2}-\left\{q_{1}, q_{2}\right\}\right) \times I$ given by $\lambda_{0}(t)=(\lambda(t), t)$. Let $\lambda_{1}$ and $\lambda_{2}$ be the strings obtained by the inclusions of $q_{1} \times I$ and $q_{2} \times I$ into $D^{2} \times I$, respectively. Then we obtain a braid of 3 strings over $D^{2}$. By adding the trivial string over $q_{3}$, we obtain a braid $\beta(w)$ of 4 strings over $S^{2}$. Observe that the map $d_{0}$ in the exact sequence above is obtained by deleting the first string. From the exact sequence above, the function $w \mapsto \beta(w)$ identifies the words in the free group $F\left(\hat{z}_{0}, \hat{z}_{1}\right)$ with the braids $\beta$ of 4 strings over $S^{2}$ that satisfy the condition that $d_{0} \beta=1$. Since the generators for $\operatorname{Brun}_{4}\left(S^{2}\right)$ are explicitly given as words in $F\left(\hat{z}_{0}, \hat{z}_{1}\right)$, it suffices 
to describe $\hat{z}_{0}$ and $\hat{z}_{1}$. Let $\sigma_{i}$ be the standard geometric braid over $D^{2}$ that has one crossing at positions $q_{i}$ and $q_{i+1}$ and trivial strings at the other positions. Recall that $\pi_{1}\left(B\left(D^{2}, n+1\right)\right)$ is generated by $\sigma_{i}$ for $0 \leq i \leq n-1$, and

$$
\pi_{1}\left(B\left(D^{2}, n+1\right)\right) \longrightarrow \pi_{1}\left(B\left(S^{2}, n+1\right)\right)
$$

is surjective by a classical result of Fadell and Van Buskirk 6, Theorem 1.11].

Write $\delta_{i}$ for the image of $\sigma_{i}$ in $\pi_{1}\left(B\left(S^{2}, n+1\right)\right)$. Then $\beta\left(\hat{z}_{0}\right)=\delta_{0}^{2}$; that is, it is obtained from the canonical generator for the pure braid on the first two positions by adding trivial strings elsewhere. Now the braid $\beta\left(\hat{z}_{1}\right)$ is obtained from a loop in $D^{2}-Q_{2}$ around $q_{2}$ avoiding $q_{1}$. Thus $\beta\left(\hat{z}_{1}\right)=\delta_{0} \delta_{1}^{2} \delta_{0}^{-1}$. (More generally, $\beta\left(\hat{z}_{k}\right)=\delta_{0} \delta_{1} \cdots \delta_{k-1} \delta_{k}^{2} \delta_{k-1}^{-1} \cdots \delta_{1}^{-1} \delta_{0}^{-1}$.) The next proposition follows.

Proposition 7.2.2. The Brunnian group $\operatorname{Brun}_{4}\left(S^{2}\right)$ is the free group of rank 5 generated by the braids $\delta_{0}^{4}, \delta_{0} \delta_{1}^{4} \delta_{0}^{-1}, \delta_{0}^{-1} \delta_{1}^{-2} \delta_{0}^{2} \delta_{1}^{2} \delta_{0}^{-1}, \delta_{0} \delta_{1}^{-2} \delta_{0}^{4} \delta_{1}^{2} \delta_{0}^{-1}$ and $\delta_{0}^{-1} \delta_{1}^{4} \delta_{0}$.

The pictures of these braids are as follows.

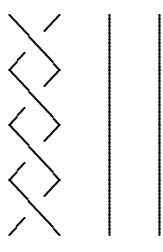

$\delta_{0}^{4}$

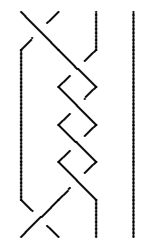

$\delta_{0} \delta_{1}^{4} \delta_{0}^{-1}$

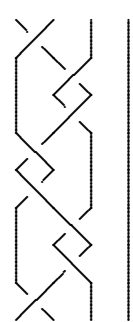

$\delta_{0}^{-1} \delta_{1}^{-2} \delta_{0}^{2} \delta_{1}^{2} \delta_{0}^{-1}$

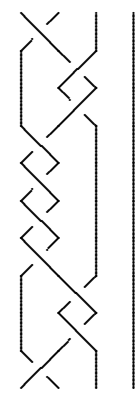

$\delta_{0} \delta_{1}^{-2} \delta_{0}^{4} \delta_{1}^{2} \delta_{0}^{-1}$

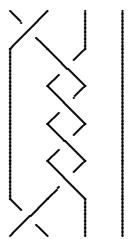

$\delta_{0}^{-1} \delta_{1}^{4} \delta_{0}$

Remark 7.2.3. By deleting the last trivial string of the 4 -string braid $\delta_{0}^{-1} \delta_{1}^{-2} \delta_{0}^{2} \delta_{1}^{2} \delta_{0}^{-1}$ over $S^{2}$ we obtain the 3 -string braid $\sigma_{0}^{-1} \sigma_{1}^{-2} \sigma_{0}^{2} \sigma_{1}^{2} \sigma_{0}^{-1}$ over $D^{2}$. In turn, closing up this 3-string braid gives a link that is readily seen to be the Borromean rings. This link corresponds to the element $\left[\hat{z}_{0}, \hat{z}_{1}\right]$ in $\hat{F}_{3}$ which can be checked, using Proposition 6.1.2, to be a Moore cycle in $F\left[S^{1}\right]_{2}$ that represents the generator $\eta_{2}$ for $\pi_{2}\left(\Omega S^{2}\right)=\pi_{3}\left(S^{2}\right)$ [40. In other words, the Hopf map $\eta_{2}: S^{3} \rightarrow S^{2}$ corresponds to the Borromean rings in this way.

7.3. The Brunnian groups $\operatorname{Brun}_{n}\left(D^{2}\right)$ for $n \leq 4$ and relations between $\operatorname{Brun}_{n}\left(D^{2}\right)$ and $\operatorname{Brun}_{n}\left(S^{2}\right)$ in low-dimensional cases. For $n=2,3,4$ the sequence of Theorem 1.2 fails to be exact. In fact, not all maps need be defined, as the following computations show.

\begin{tabular}{|c|c|c|c|c|c|c|c|c|}
\hline $\operatorname{Brun}_{3}\left(S^{2}\right)$ & $\rightarrow$ & $\operatorname{Brun}_{2}\left(D^{2}\right)$ & $\rightarrow$ & $\operatorname{Brun}_{2}\left(S^{2}\right)$ & $\rightarrow$ & Coker & $\leftarrow$ & $\pi_{1}\left(S^{2}\right)$ \\
\hline 11 & & 11 & & 11 & & 11 & & II \\
\hline $\mathbb{Z} / 2$ & & $\mathbb{Z}$ & & $\mathbb{Z} / 2$ & & 1 & & 1 \\
\hline $\operatorname{Brun}_{4}\left(S^{2}\right)$ & $\rightarrow$ & $\operatorname{Brun}_{3}\left(D^{2}\right)$ & $\rightarrow$ & $\operatorname{Brun}_{3}\left(S^{2}\right)$ & $\rightarrow$ & Coker & $\leftarrow$ & $\pi_{2}\left(S^{2}\right)$ \\
\hline II & & 11 & & 11 & & 11 & & 11 \\
\hline$F_{5}$ & & {$\left[F_{2}, F_{2}\right]=F_{\omega}$} & & $\mathbb{Z} / 2$ & & $\mathbb{Z} / 2$ & & $\mathbb{Z}$ \\
\hline $\operatorname{Brun}_{5}\left(S^{2}\right)$ & $\hookrightarrow$ & $\operatorname{Brun}_{4}\left(D^{2}\right)$ & $\rightarrow$ & $\operatorname{Brun}_{4}\left(S^{2}\right)$ & $\rightarrow$ & Coker & $\leftarrow$ & $\pi_{3}\left(S^{2}\right)$ \\
\hline 11 & & 11 & & 11 & & 11 & & 11 \\
\hline$F_{\omega}$ & & $F_{\omega}$ & & $F_{5}$ & & $\pi_{3}\left(\mathcal{E}^{\pi_{1}}\right)$ & & $\mathbb{Z}$ \\
\hline
\end{tabular}


where $\pi_{3}\left(\mathcal{E}^{\pi_{1}}\right)$ is shown in Proposition 7.1 .1 to be nilpotent of class 2. It follows from the proof of Theorem 1.2 that the sequence

$$
\operatorname{Brun}_{5}\left(S^{2}\right) \longmapsto \operatorname{Brun}_{4}\left(D^{2}\right) \longrightarrow \operatorname{Brun}_{4}\left(S^{2}\right)
$$

is exact. A determination of a free basis for $\operatorname{Brun}_{4}\left(D^{2}\right)$ is given below, where the terminology is as in Subsection 6.3.

Recall that

$$
\operatorname{Brun}_{4}\left(D^{2}\right)=\bigcap_{j=0}^{3} \operatorname{Ker}\left(d_{i}: P_{4} \rightarrow P_{3}\right) .
$$

Write $\operatorname{Ker}\left(d_{i}\right)$ for $\operatorname{Ker}\left(d_{i}: P_{4} \rightarrow P_{3}\right)$ and recall that $F\left(\mathbb{R}^{2}, n\right) \simeq F\left(D^{2}, n\right)$. From the fibration

$$
\mathbb{R}^{2} \backslash Q_{3} \longrightarrow F\left(\mathbb{R}^{2}, 4\right) \stackrel{d_{0}}{\longrightarrow} F\left(\mathbb{R}^{2}, 3\right)
$$

$\operatorname{Ker}\left(d_{0}\right)$ is the free subgroup of $P_{4}$ generated by $A_{0,1}, A_{0,2}, A_{0,3}$. Let $x_{i}$ denote $A_{0, i}$ for $i=1,2,3$. Observe that there is a commutative diagram

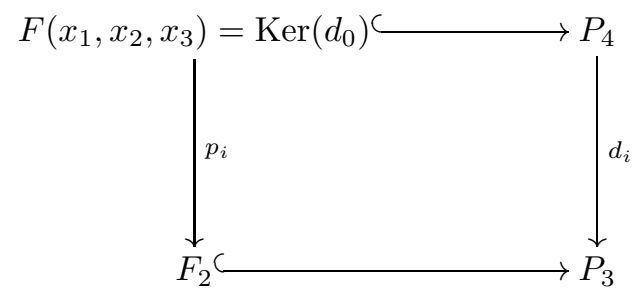

for $i=1,2,3$, where $p_{i}: F\left(x_{1}, x_{2}, x_{3}\right) \rightarrow F_{2}$ is the projection map sending $x_{i}$ to 1 and preserving the other generators.

1. The subgroup $\operatorname{Ker}\left(d_{0}\right) \cap \operatorname{Ker}\left(d_{1}\right)=\operatorname{Ker}\left(p_{1}: F_{3} \rightarrow F_{2}\right)$.

Since $p_{1}: F\left(x_{1}, x_{2}, x_{3}\right) \rightarrow F\left(x_{2}, x_{3}\right)$ is the projection map, $\operatorname{Ker}\left(p_{1}\right)$ is freely generated by the set of iterated commutators

$$
A=\left\{x_{1},\left[\left[\left[x_{1}, x_{i_{1}}^{\epsilon_{1}}\right], x_{i_{2}}^{\epsilon_{2}}\right], \ldots, x_{i_{t}}^{\epsilon_{t}}\right] \mid w=x_{i_{1}}^{\epsilon_{1}} x_{i_{2}}^{\epsilon_{2}} \cdots x_{i_{t}}^{\epsilon_{t}} \text { a reduced word in } F\left(x_{2}, x_{3}\right)\right\}
$$

by [70, Proposition 3.3]. (Our notation is always that $\epsilon_{i}, \epsilon_{i}^{\prime} \in\{ \pm 1\}$.)

2. The subgroup $\operatorname{Ker}\left(d_{0}\right) \cap \operatorname{Ker}\left(d_{1}\right) \cap \operatorname{Ker}\left(d_{2}\right)=\operatorname{Ker}\left(p_{1}\right) \cap \operatorname{Ker}\left(p_{2}\right)$.

Let

$$
\begin{gathered}
A_{1}=\left\{\alpha \in A \mid x_{2} \text { occurs in } \alpha\right\}, \\
A_{2}=\left\{\alpha \in A \mid x_{2} \text { does not occur in } \alpha\right\} .
\end{gathered}
$$

Then $A=A_{1} \coprod A_{2}$. Since $p_{2}: F\left(x_{1}, x_{2}, x_{3}\right) \rightarrow F\left(x_{1}, x_{3}\right)$ is the projection map, $p_{2}(\alpha)=\alpha \in F\left(x_{1}, x_{3}\right)$ if $\alpha \in A_{2}$ and $p_{2}(\alpha)=1$ if $\alpha \in A_{1}$. This gives the commutative diagram

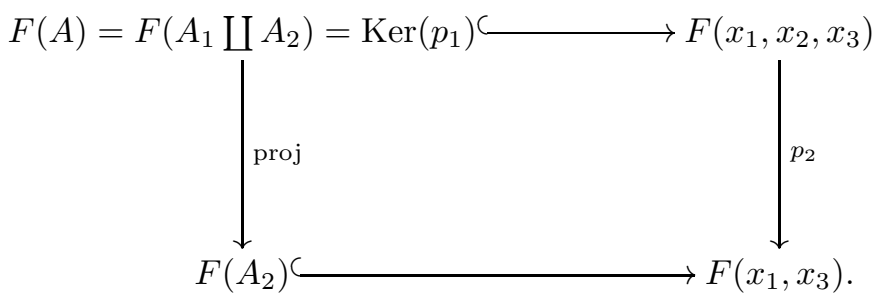


By [70, Proposition 3.3], the subgroup $\operatorname{Ker}\left(p_{1}\right) \cap \operatorname{Ker}\left(p_{2}\right)$ is freely generated by the set

$B=\left\{\alpha_{1},\left[\left[\alpha_{1}, \alpha_{i_{1}}^{\epsilon_{1}}\right], \ldots, \alpha_{i_{t}}^{\epsilon_{t}}\right] \mid \alpha_{1} \in A_{1}, w=\alpha_{i_{1}}^{\epsilon_{1}} \alpha_{i_{2}}^{\epsilon_{2}} \cdots \alpha_{i_{t}}^{\epsilon_{t}}\right.$ a reduced word in $\left.F\left(A_{2}\right)\right\}$.

3. The subgroup $\bigcap_{j=0}^{3} \operatorname{Ker}\left(d_{j}\right)=\bigcap_{j=1}^{3} \operatorname{Ker}\left(p_{j}\right)$.

Observe that $p_{3}: F\left(x_{1}, x_{2}, x_{3}\right) \rightarrow F\left(x_{1}, x_{3}\right)$. Similarly to the above, let

$$
\begin{gathered}
B_{1}=\left\{\beta \in B \mid x_{3} \text { occurs in } \beta\right\}, \\
B_{2}=\left\{\beta \in B \mid x_{3} \text { does not occur in } \beta\right\} .
\end{gathered}
$$

Then a free basis for $\operatorname{Brun}_{4}\left(D^{2}\right)=\bigcap_{j=0}^{3} \operatorname{Ker}\left(d_{j}\right)=\bigcap_{j=1}^{3} \operatorname{Ker}\left(p_{j}\right)$ is given by $C=\left\{\beta_{1},\left[\left[\beta_{1}, \beta_{i_{1}}^{\epsilon_{1}}\right], \ldots, \beta_{i_{t}}^{\epsilon_{t}}\right] \mid \beta_{1} \in B_{1}, w=\beta_{i_{1}}^{\epsilon_{1}} \beta_{i_{2}}^{\epsilon_{2}} \ldots \beta_{i_{t}}^{\epsilon_{t}}\right.$ a reduced word in $\left.F\left(B_{2}\right)\right\}$.

(Note. A free basis for general $\operatorname{Brun}_{n}\left(D^{2}\right)$ can be given recursively as described above. Detailed discussions for intersection subgroups of projection maps were given in [70, Theorem 3.5].) Let

$$
\operatorname{ad}^{n}(b)(a)= \begin{cases}{[[a, \overbrace{b], \ldots, b]}^{n}} & n>0, \\ a & n=0, \\ {\left[[a \overbrace{\left.b^{-1}\right], \ldots, b^{-1}}^{n-n}\right.} & n<0 .\end{cases}
$$

Then $A_{2}=\left\{\operatorname{ad}^{n}\left(x_{3}\right)\left(x_{1}\right) \mid n \in \mathbb{Z}\right\}$ and so the set $B$ consists of the elements:

(1) $\alpha=\left[\left[x_{1}, x_{l_{1}}^{\epsilon_{1}}\right], \ldots, x_{l_{t}}^{\epsilon_{t}}\right]$ such that $w=x_{l_{1}}^{\epsilon_{1}} \cdots x_{l_{t}}^{\epsilon_{t}}$ is a reduced word in $F\left(x_{2}, x_{3}\right)$ with $l_{r}=2$ where $1 \leq r \leq t$, and

(2) $\left[\left[\alpha,\left(\operatorname{ad}^{n_{1}}\left(x_{3}\right)\left(x_{1}\right)\right)^{\epsilon_{1}}\right], \ldots,\left(\operatorname{ad}^{n_{k}}\left(x_{3}\right)\left(x_{1}\right)\right)^{\epsilon_{k}}\right]$ for $\alpha$ as above and reduced words $\left(\operatorname{ad}^{n_{1}}\left(x_{3}\right)\left(x_{1}\right)\right)^{\epsilon_{1}} \cdots\left(\operatorname{ad}^{n_{k}}\left(x_{3}\right)\left(x_{1}\right)\right)^{\epsilon_{k}} \in F\left(\operatorname{ad}^{n}\left(x_{3}\right)\left(x_{1}\right) \mid n \in \mathbb{Z}\right)$ excluding the identity 1 .

The set $B_{2}$ is given by

$$
B_{2}=\left\{\operatorname{ad}^{t}\left(x_{1}\right)\left(\operatorname{ad}^{s}\left(x_{2}\right)\left(x_{1}\right)\right) \mid s, t \in \mathbb{Z}, s \neq 0 \text { if } t \neq 0\right\},
$$

while $B_{1}$ takes the remaining elements of $B$. Thus the set $C$ consists of the following types of elements:

(C1) the commutators

$$
\alpha=\left[\left[x_{1}, x_{l_{1}}^{\epsilon_{1}}\right], \ldots, x_{l_{t}}^{\epsilon_{t}}\right]
$$

for reduced words $w=x_{l_{1}}^{\epsilon_{1}} \cdots x_{l_{t}}^{\epsilon_{t}} \in F\left(x_{2}, x_{3}\right)$ such that both $x_{2}$ and $x_{3}$ occur in $w$;

(C2) the commutators

$$
\beta=\left[\left[\alpha,\left(\operatorname{ad}^{t_{1}}\left(x_{1}\right)\left(\operatorname{ad}^{s_{1}}\left(x_{2}\right)\left(x_{1}\right)\right)^{\epsilon_{1}}\right], \ldots,\left(\operatorname{ad}^{t_{k}}\left(x_{1}\right)\left(\operatorname{ad}^{s_{k}}\left(x_{2}\right)\left(x_{1}\right)\right)\right)^{\epsilon_{k}}\right]\right.
$$

for $\alpha$ as in (C1) above and reduced words

$$
\left(\operatorname{ad}^{t_{1}}\left(x_{1}\right)\left(\operatorname{ad}^{s_{1}}\left(x_{2}\right)\left(x_{1}\right)\right)\right)^{\epsilon_{1}} \cdots\left(\operatorname{ad}^{t_{k}}\left(x_{1}\right)\left(\operatorname{ad}^{s_{k}}\left(x_{2}\right)\left(x_{1}\right)\right)\right)^{\epsilon_{k}}
$$

in $F\left(\operatorname{ad}^{t}\left(x_{1}\right)\left(\operatorname{ad}^{s}\left(x_{2}\right)\left(x_{1}\right)\right) \mid s, t \in \mathbb{Z}, s \neq 0\right.$ if $\left.t \neq 0\right)$ excluding the identity 1; 
(C3) the commutators

$$
\gamma=\left[\left[\left[\left[x_{1}, x_{l_{1}}^{\epsilon_{1}}\right], \ldots, x_{l_{t}}^{\epsilon_{t}}\right],\left(\operatorname{ad}^{n_{1}}\left(x_{3}\right)\left(x_{1}\right)\right)^{\epsilon_{1}^{\prime}}\right], \ldots,\left(\operatorname{ad}^{n_{k}}\left(x_{3}\right)\left(x_{1}\right)\right)^{\epsilon_{k}^{\prime}}\right]
$$

for reduced words $x_{l_{1}}^{\epsilon_{1}} \cdots x_{l_{t}}^{\epsilon_{t}} \in F\left(x_{2}, x_{3}\right)$ with $x_{2}$ occurring, and reduced words $\left(\operatorname{ad}^{n_{1}}\left(x_{3}\right)\left(x_{1}\right)\right)^{\epsilon_{1}^{\prime}} \cdots\left(\operatorname{ad}^{n_{k}}\left(x_{3}\right)\left(x_{1}\right)\right)^{\epsilon_{k}^{\prime}} \in F\left(\operatorname{ad}^{n}\left(x_{3}\right)\left(x_{1}\right) \mid n \in \mathbb{Z}\right)$ with $x_{3}$ occurring;

(C4) the commutators

$$
\delta=\left[\left[\gamma,\left(\operatorname{ad}^{t_{1}}\left(x_{1}\right)\left(\operatorname{ad}^{s_{1}}\left(x_{2}\right)\left(x_{1}\right)\right)^{\epsilon_{1}}\right], \ldots,\left(\operatorname{ad}^{t_{k}}\left(x_{1}\right)\left(\operatorname{ad}^{s_{k}}\left(x_{2}\right)\left(x_{1}\right)\right)\right)^{\epsilon_{k}}\right]\right.
$$

for $\gamma$ as in (C3) above and reduced words

$$
\left(\operatorname{ad}^{t_{1}}\left(x_{1}\right)\left(\operatorname{ad}^{s_{1}}\left(x_{2}\right)\left(x_{1}\right)\right)\right)^{\epsilon_{1}} \cdots\left(\operatorname{ad}^{t_{k}}\left(x_{1}\right)\left(\operatorname{ad}^{s_{k}}\left(x_{2}\right)\left(x_{1}\right)\right)\right)^{\epsilon_{k}}
$$

in $F\left(\operatorname{ad}^{t}\left(x_{1}\right)\left(\operatorname{ad}^{s}\left(x_{2}\right)\left(x_{1}\right)\right) \mid s, t \in \mathbb{Z}, s \neq 0\right.$ if $\left.t \neq 0\right)$ excluding the identity 1.

7.4. The 5- and 6-strand Brunnian braids. Examples for constructing 5 and 6 -strand Brunnian braids are given in this subsection. The method here is to use the simplicial operations described below.

Let $\Delta[n]$ be the standard simplicial $n$-simplex with the (only) $n$-dimensional nondegenerate element $\sigma_{n} \in(\Delta[n])_{n}$. Let $\mathcal{X}$ be a simplicial set and let $x \in X_{n}$ be any $n$-dimensional element. As in [21], there is a (unique) simplicial map $f_{x}: \Delta[n] \rightarrow X$ such that $f_{x}\left(\sigma_{n}\right)=x$. Recall that the simplicial $n$-sphere $S^{n}$ is the quotient simplicial set of $\Delta[n]$ obtained by identifying all faces of $\sigma_{n}$ to the basepoint. Let $q: \Delta[n] \rightarrow S^{n}$ be the quotient map and let $\bar{\sigma}_{n}=q\left(\sigma_{n}\right)$.

Now, given a simplicial group $\mathcal{G}$ with a Moore cycle $w \in \mathrm{Z}_{n} \mathcal{G}$, consider the representing map $f_{w}: \Delta[n] \rightarrow \mathcal{G}$ with $f_{w}\left(\sigma_{n}\right)=w$. Since $d_{i} w=1$ for all $i$, the map $f_{w}$ factors through the quotient simplicial set $S^{n}$. Let $\bar{f}_{w}: S^{n} \rightarrow \mathcal{G}$ be the simplicial map such that $f_{w}=\bar{f}_{w} \circ q$. By applying the universal property of Milnor's construction, the map $\bar{f}_{w}: S^{n} \rightarrow \mathcal{G}$ extends to a (unique) simplicial homomorphism

$$
F\left(\bar{f}_{w}\right): F\left[S^{n}\right] \rightarrow \mathcal{G} .
$$

For $\alpha \in \mathrm{Z}_{n+k} F\left[S^{n}\right]$, write $w \odot \alpha$ for the Moore cycle $F\left(\bar{f}_{w}\right)(\alpha) \in \mathrm{Z}_{n+k} \mathcal{G}$. The homotopy class in $\pi_{n+k}(\mathcal{G})$ represented by $w \odot \alpha$ is the usual composition operation in homotopy theory.

Consider the case where $k=1$. Recall that $\pi_{2}\left(F\left[S^{1}\right]\right)=\pi_{3}\left(S^{2}\right)=\mathbb{Z}$ and $\pi_{n+1}\left(F\left[S^{n}\right]\right)=\pi_{n+2}\left(S^{n+1}\right)=\mathbb{Z} / 2$ for $n>1$. After [67], the generator for $\pi_{n+2}\left(S^{n+1}\right), n \geq 1$, is denoted by $\eta_{n+1}$. According to [70, Example 2.23], the homotopy class $\eta_{n+1}$ is represented by the cycle

$$
\left[s_{0} \bar{\sigma}_{n}, s_{1} \bar{\sigma}_{n}\right] \in \mathrm{Z}_{n+1} F\left[S^{n}\right]
$$

with the relations

$$
\begin{cases}{\left[s_{i} \bar{\sigma}_{n}, s_{i+1} \bar{\sigma}_{n}\right] \equiv\left[s_{0} \bar{\sigma}_{n}, s_{1} \bar{\sigma}_{n}\right]} & \text { if } 0 \leq i \leq n-1, \\ {\left[s_{i} \bar{\sigma}_{n}, s_{j} \bar{\sigma}_{n}\right] \equiv 1} & \text { if } i+1<j\end{cases}
$$

where $a \equiv b$ means that $a$ and $b$ represent the same element in the homotopy group. By Proposition 6.1.2, the cycle $\left[\hat{z}_{0}, \hat{z}_{1}\right] \equiv\left[s_{0} \bar{\sigma}_{1}, s_{1} \bar{\sigma}_{1}\right]$ also represents the generator $\eta_{2} \in \pi_{2}(\hat{F})=\pi_{2}\left(F\left[S^{1}\right]\right)=\mathbb{Z}$.

Recall that $\pi_{3}\left(F\left[S^{1}\right]\right)=\pi_{4}\left(S^{2}\right)=\mathbb{Z} / 2$ and $\pi_{4}\left(F\left[S^{1}\right]\right)=\pi_{5}\left(S^{2}\right)=\mathbb{Z} / 2$ are generated by $\eta_{2}^{2}$ and $\eta_{2}^{3}$ respectively; see [67]. By using the composition method 
described above, $\eta_{2}^{2}$ corresponds to the element

$$
\begin{aligned}
\alpha_{5} & =\left[\hat{z}_{0}, \hat{z}_{1}\right] \odot\left[s_{0} \bar{\sigma}_{2}, s_{1} \bar{\sigma}_{2}\right]=\left[s_{0}\left[\hat{z}_{0}, \hat{z}_{1}\right], s_{1}\left[\hat{z}_{0}, \hat{z}_{1}\right]\right] \\
& =\left[\left[s_{0} \hat{z}_{0}, s_{0} \hat{z}_{1}\right],\left[s_{1} \hat{z}_{0}, s_{1} \hat{z}_{1}\right]\right]=\left[\left[\hat{z}_{0} \hat{z}_{1}, \hat{z}_{2}\right],\left[\hat{z}_{0}, \hat{z}_{1} \hat{z}_{2}\right]\right]
\end{aligned}
$$

in $\hat{F}_{4}$, mapping under the map $\beta$ defined before Proposition 7.2 .2 to the 5 -string Brunnian braid over $S^{2}$ :

$$
\left[\left[\delta_{0}^{3} \delta_{1}, \delta_{0} \delta_{1} \delta_{2}^{2} \delta_{1}^{-1} \delta_{0}^{-1}\right],\left[\delta_{0}^{2}, \delta_{0} \delta_{1}^{3} \delta_{2}^{2} \delta_{1}^{-1} \delta_{0}^{-1}\right]\right] .
$$

Likewise, $\eta_{2}^{3}$ corresponds to the element

$$
\begin{aligned}
\alpha_{6} & =\alpha_{5} \odot\left[s_{0} \bar{\sigma}_{3}, s_{1} \bar{\sigma}_{3}\right]=\left[s_{0} \alpha_{5}, s_{1} \alpha_{5}\right] \\
& =\left[\left[\left[\hat{z}_{0} \hat{z}_{1} \hat{z}_{2}, \hat{z}_{3}\right],\left[\hat{z}_{0} \hat{z}_{1}, \hat{z}_{2} \hat{z}_{3}\right]\right],\left[\left[\hat{z}_{0} \hat{z}_{1} \hat{z}_{2}, \hat{z}_{3}\right],\left[\hat{z}_{0}, \hat{z}_{1} \hat{z}_{2} \hat{z}_{3}\right]\right]\right] .
\end{aligned}
$$

By Theorem 1.2. the 5- and 6-strand braids above represent the only nontrivial Brunnian braids over $S^{2}$ modulo Brunnian braids over $D^{2}$ in those dimensions.

By Equation (18), the element

$$
\begin{aligned}
\alpha_{5}^{\prime} & =\left[\hat{z}_{0}, \hat{z}_{1}\right] \odot\left[s_{1} \bar{\sigma}_{2}, s_{2} \bar{\sigma}_{2}\right]=\left[s_{1}\left[\hat{z}_{0}, \hat{z}_{1}\right], s_{2}\left[\hat{z}_{0}, \hat{z}_{1}\right]\right] \\
& =\left[\left[s_{1} \hat{z}_{0}, s_{1} \hat{z}_{1}\right],\left[s_{2} \hat{z}_{0}, s_{2} \hat{z}_{1}\right]\right]=\left[\left[\hat{z}_{0}, \hat{z}_{1} \hat{z}_{2}\right],\left[\hat{z}_{0}, \hat{z}_{1}\right]\right]
\end{aligned}
$$

also represents the nontrivial 5 -strand Brunnian braid over $S^{2}$ modulo Brunnian braids over $D^{2}$ as

$$
\left[\left[\delta_{0}^{2}, \delta_{0} \delta_{1}^{3} \delta_{2}^{2} \delta_{1}^{-1} \delta_{0}^{-1}\right],\left[\delta_{0}^{2}, \delta_{0} \delta_{1}^{2} \delta_{0}^{-1}\right]\right] .
$$

Likewise, $\eta_{2}^{3}$ also corresponds to the element

$$
\begin{aligned}
\alpha_{6}^{\prime} & =\alpha_{5}^{\prime} \odot\left[s_{2} \bar{\sigma}_{3}, s_{3} \bar{\sigma}_{3}\right]=\left[s_{2} \alpha_{5}^{\prime}, s_{3} \alpha_{5}^{\prime}\right] \\
& =\left[\left[\left[\hat{z}_{0}, \hat{z}_{1} \hat{z}_{2} \hat{z}_{3}\right],\left[\hat{z}_{0}, \hat{z}_{1}\right]\right],\left[\left[\hat{z}_{0}, \hat{z}_{1} \hat{z}_{2}\right],\left[\hat{z}_{0}, \hat{z}_{1}\right]\right]\right] .
\end{aligned}
$$

By [67, the element $\eta_{2}^{4}$ is a nontrivial element divisible by 6 in the homotopy group $\pi_{6}\left(S^{2}\right)=\mathbb{Z} / 12$, and $\eta_{2}^{5}=0 \in \pi_{7}\left(S^{2}\right)=\mathbb{Z} / 2$. Thus

$$
\begin{gathered}
\alpha_{7}^{\prime}=\alpha_{6}^{\prime} \odot\left[s_{3} \bar{\sigma}_{4}, s_{4} \bar{\sigma}_{4}\right]=\left[s_{3} \alpha_{6}^{\prime}, s_{4} \alpha_{6}\right]= \\
{\left[\left[\left[\left[\hat{z}_{0}, \hat{z}_{1} \hat{z}_{2} \hat{z}_{3} \hat{z}_{4}\right],\left[\hat{z}_{0}, \hat{z}_{1}\right]\right],\left[\left[\hat{z}_{0}, \hat{z}_{1} \hat{z}_{2}\right],\left[\hat{z}_{0}, \hat{z}_{1}\right]\right]\right],\left[\left[\left[\hat{z}_{0}, \hat{z}_{1} \hat{z}_{2} \hat{z}_{3}\right],\left[\hat{z}_{0}, \hat{z}_{1}\right]\right],\left[\left[\hat{z}_{0}, \hat{z}_{1} \hat{z}_{2}\right],\left[\hat{z}_{0}, \hat{z}_{1}\right]\right]\right]\right]}
\end{gathered}
$$

represents a nontrivial Brunnian 7-strand over $S^{2}$ modulo Brunnian braids over $D^{2}$, and $\bar{\alpha}$ is divisible by 6 modulo Brunnian braids over $D^{2}$. In each case above, twice the element obtained represents a Brunnian braid over $D^{2}$. This algorithm stops at the next dimension, where

$$
\alpha_{8}^{\prime}=\alpha_{7}^{\prime} \odot\left[s_{4} \bar{\sigma}_{5}, s_{5} \bar{\sigma}_{5}\right]=\left[s_{4} \alpha_{7}^{\prime}, s_{5} \alpha_{7}^{\prime}\right]
$$

represents the trivial 8-strand braid over $S^{2}$ modulo Brunnian braids over $D^{2}$. According to 67, $\pi_{7}\left(S^{2}\right)=\mathbb{Z} / 2$ is generated by $\nu^{\prime} \circ \eta_{7}$, and so the nontrivial Brunnian 8-strand braid over $S^{2}$ modulo Brunnian braids over $D^{2}$ is represented by

$$
\beta_{8}=\beta_{7} \odot\left[s_{4} \bar{\sigma}_{5}, s_{5} \bar{\sigma}_{5}\right]=\left[s_{4} \beta_{7}, s_{5} \beta_{7}\right],
$$

where $\beta_{7}$ is a cycle in $\hat{F}_{6}$ representing the generator $\nu^{\prime}$ for the 2-primary part of $\pi_{6}\left(S^{2}\right)=\mathbb{Z} / 12$. So far, explicit simplicial constructions for $\beta_{7}$ and the generator of $\pi_{6}\left(S^{2}\right)$ are elusive.

As noted in the Introduction, closing up a Brunnian braid gives a Brunnian link. For example, $\eta_{2}$ gives the Borromean rings; see Subsection 7.2 It would 
be interesting to see Brunnian links corresponding to the other elements exhibited above.

\section{REMARKS}

8.1. Notation. The diagram

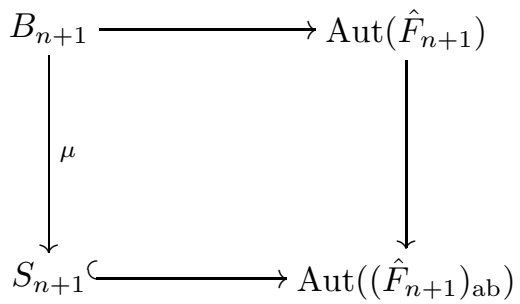

of Lemma 6.3.5 highlights some notational choices that must be made. The original convention of Artin [2] may be portrayed as

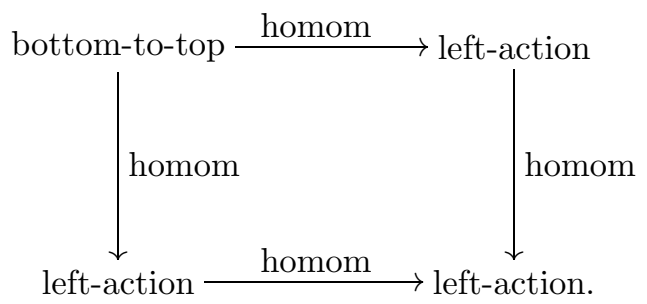

On the other hand, Birman's convention $[6]$ is precisely the opposite:

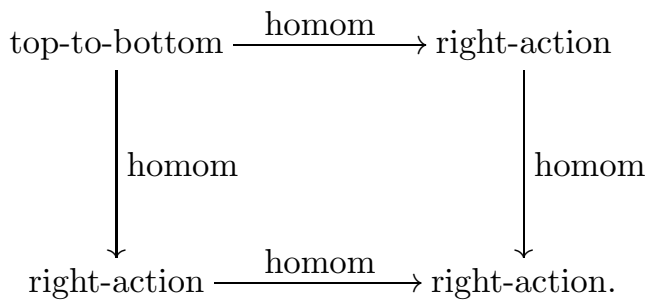

The following hybrid notation has been adopted in this article, representing the natural composition of braids from top to bottom (so that $\alpha$ above $\beta$ is written $\alpha \beta$ ). This choice of notation also uses the conventional left-action of matrices, giving rise to anti-homomorphisms as follows:

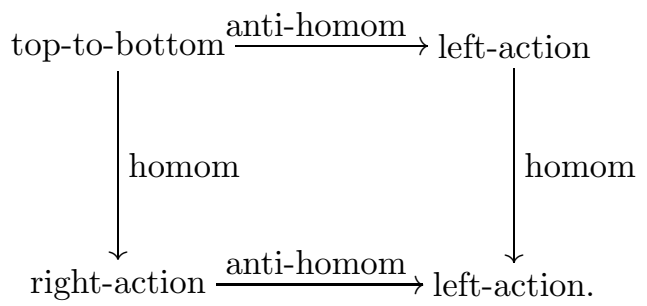


8.2. Birman's problem. The group of pure braids of $n$ strings whose reduced Artin representations are inner automorphisms of $\hat{F}_{n}$, called $R_{n}$ in this article, is written as $R$ in Birman's book [6, pp. 133-136]. Then Birman defines an explicit subgroup $R_{n-1}^{\mathrm{Bir}}$ of $P_{n}$ such that $R_{n}=\mathcal{Z}\left(P_{n}\right) \times R_{n-1}^{\mathrm{Bir}}$. The notation here is slightly different from Birman's. In [6, Problem 23, p.219], Birman then posted a research problem:

(1). Find a free basis for the group $\bigcap_{k=1}^{n}\left(\operatorname{Ker} \pi_{*}^{k} \cap R_{n-1}^{\mathrm{Bir}}\right)$.

According to [6, p.138], $\pi_{*}^{k}$ is induced by the map $\pi^{k}: F\left(D^{2}, n\right) \rightarrow F\left(D^{2}, n-1\right)$ defined by

$$
\pi^{k}\left(z_{1}, \ldots, z_{n}\right)=\left(z_{1}, \ldots, z_{k-1}, z_{k+1}, \ldots, z_{n}\right) .
$$

According to the terminology here, $\pi_{*}^{k}=d_{k-1}$ are exactly the faces on $\mathcal{P}$. By Corollary 6.3.7 there are isomorphisms

$$
\bigcap_{k=1}^{n}\left(\operatorname{Ker} \pi_{*}^{k} \cap R_{n-1}^{\mathrm{Bir}}\right) \cong \mathrm{Z}_{n-1}\left(F\left[S^{1}\right]\right) \cong \operatorname{Brun}_{n+1}\left(S^{2}\right)
$$

for $n \geq 4$. Thus Birman's problem is equivalent to finding a free basis for $\mathrm{Z}\left(F\left[S^{1}\right]\right)$. The solution of this problem will yield a combinatorial presentation of the higher homotopy groups of $S^{2}$ because a set of generators for $\mathrm{B}\left(F\left[S^{1}\right]\right)$ has been determined in 70 .

8.3. Linear representation of the braid groups. As reported in 6, pp. 138143], Birman's problem above arose from the Gassner representation of $P_{n}$, which is a linear representation of pure braids. The interpretation of [6, Theorem 3.18] in the terminology here is that the sequence of the Gassner matrix groups is a $\Delta$-group and the Gassner representation is a morphism of $\Delta$-groups. By using the Gassner representation, the Moore cycles $\mathrm{Z}\left(F\left[S^{1}\right]\right)$ admit linear representations. Under current technology, it is unclear whether the homotopy group $\pi_{n}\left(F\left[S^{1}\right]\right)$ can be described as a subquotient of the Gassner matrix group; however, this deserves study. Recently Krammer and Bigelow [5, 47] proved that the braid group admits a faithful representation into a general linear group over the reals, which can provide helpful information for studying homotopy groups using linear representations.

8.4. Artin's representation. Let $\mathcal{P}=\left\{P_{n+1}\right\}_{n \geq 0}$ be the sequence of the Artin pure braid group with the simplicial group structure given in Theorem 3.2.12. Let $\hat{F}=\left\{\hat{F}_{n+1}\right\}_{n \geq 0}$ be the simplicial group described in Subsection 6.3. By Lemma 6.3.1, the classical Artin representation admits a simplicial interpretation; namely, the Artin representation induces a simplicial action

$$
\mu: \mathcal{P} \times \hat{F} \longrightarrow \hat{F} .
$$

The action $\mu$ does not directly give homotopy information in geometry because the space $\mathcal{P}$ is contractible. However, modulo Moore boundaries in $\hat{F}$, the Artin representation induces an action

$$
\bar{\mu}: \mathcal{P} \times \hat{F} / \mathrm{B} \hat{F} \longrightarrow \hat{F} / \mathrm{B} \hat{F} .
$$

The action $\bar{\mu}$ does give homotopy information in the sense that:

Theorem 8.4.1. [71, Theorem 1.2] For $n \geq 3$, the fixed set of the $P_{n+1}$-action on $\hat{F}_{n+1} / \mathrm{B}_{n} \hat{F}$ is isomorphic to the homotopy group $\pi_{n+1}\left(S^{3}\right)$. 
Another remark concerning the simplicial action $\mu: \mathcal{P} \times \hat{F} \rightarrow \hat{F}$ is that, for each $\alpha \in \mathcal{P}$, the function $\rho: \hat{F} \rightarrow \hat{F}, \quad x \mapsto \mu(\alpha, x) x^{-1}$ maps $\Gamma_{t} \hat{F}$ into $\Gamma_{t+1} \hat{F}$, where $\left\{\Gamma_{t} \hat{F}\right\}_{t \geq 1}$ is the (mod $p$ or integral) descending central series of $\hat{F}$, and therefore $\rho$ induces operations on the Adams spectral sequence for $\hat{F}$ that converges to $\pi_{*}\left(\Omega S^{2}\right)$. It seems that there are connections between the operations induced by $\rho$ and higher differentials in the Adams spectral sequence.

8.5. Homotopy groups of spheres. We present here some historical context for this work. The fundamental group owes its existence to Poincaré [57. Čech [15] suggested how to define higher homotopy groups in 1932 without pursuing the notion, and it was Hurewicz [37] who first studied them in 1935-36. It was originally conjectured that the homotopy groups of spheres are isomorphic to their homology groups. Then Hopf invented the Hopf map [36]. The determination of higher homotopy groups of spheres became the fundamental problem in homotopy theory from then on. Although the determination of the general homotopy groups is beyond current technology, much progress has been made over time. By using connections with braids established in this article, some classical results on $\pi_{*}\left(S^{2}\right)$ provide certain information on braids.

Serre 64] proved that

(1) $\pi_{m}\left(S^{2 n+1}\right)$ is a finite abelian group for $m>2 n+1$;

(2) $\pi_{m}\left(S^{2 n}\right)$ is a finite group for $m>2 n>0$ and $m \neq 4 n-1$;

(3) $\pi_{4 n-1}\left(S^{2 n}\right)=\mathbb{Z} \oplus$ finite group for $n>0$.

In particular, $\pi_{r}\left(S^{2}\right)$ is a finite abelian group for $r>3$. Together with Theorem 1.2 this proves the following:

Theorem 8.5.1. For each $n \geq 5$, the cokernel of the group homomorphism

$$
\operatorname{Brun}_{n}\left(D^{2}\right) \longrightarrow \operatorname{Brun}_{n}\left(S^{2}\right)
$$

is a finite abelian group.

Let $G$ be an abelian group and let $\operatorname{Tor}_{p}(G)=\left\{x \in G \mid p^{t} x=0\right.$ for some $\left.t\right\}$. The $p$-exponent $\exp _{p}(G)$ of $G$ is defined to be

$$
\exp _{p}(G)=\min \left\{p^{r} \mid p^{r} \cdot \operatorname{Tor}_{p}(G)=0\right\}
$$

where $\exp _{p}(G)=+\infty$ if $p^{r} \cdot \operatorname{Tor}_{p}(G) \neq 0$ for every integer $r$. A result of J. C. Moore (unpublished) and James [38] is that

$$
\exp _{2}\left(\pi_{*}\left(S^{2}\right)\right)=4 \text {. }
$$

For odd primes $p$, Selick 62 proved that

$$
\exp _{p}\left(\pi_{*}\left(S^{2}\right)\right)=p
$$

By using Theorem 1.2, these results apply to braids as follows.

Theorem 8.5.2. Let $n \geq 5$ and let $\beta$ be an n-strand Brunnian braid over $S^{2}$. Then there exists an integer $r$ such that

$$
\beta^{r} \in \operatorname{Im}\left(\operatorname{Brun}_{n}\left(D^{2}\right) \rightarrow \operatorname{Brun}_{n}\left(S^{2}\right)\right) .
$$

Moreover, suppose that $r$ is the smallest positive integer such that

$$
\beta^{r} \in \operatorname{Im}\left(\operatorname{Brun}_{n}\left(D^{2}\right) \rightarrow \operatorname{Brun}_{n}\left(S^{2}\right)\right) .
$$


Then $r$ admits a decomposition

$$
r=2^{\epsilon} p_{1} p_{2} \cdots p_{t}
$$

for some $0 \leq \epsilon \leq 2$, and for some $t \geq 0$ with positive prime integers

$$
2<p_{1}<p_{2}<\cdots<p_{t} .
$$

(It follows from Subsection 7.3 that this result also holds for $n=2,3$, but fails for $n=4$.) One particular consequence is that the odd-primary torsion component of $\pi_{n}\left(S^{2}\right)$ is a vector space over $\mathbb{Z} / p$. Thus the odd-primary torsion component of $\pi_{n}\left(S^{2}\right)$ could be determined if one could determine the order of the group $\pi_{n}\left(S^{2}\right)$. This suggests a problem whether there is a group-theoretical or geometric method for determining the order of the finite group $\operatorname{Coker}\left(\operatorname{Brun}_{n}\left(D^{2}\right) \rightarrow \operatorname{Brun}_{n}\left(S^{2}\right)\right.$ ) for $n \geq 5$.

There are known families of periodic elements in $\pi_{*}\left(S^{2}\right)$. Mark Mahowald asked how to represent these elements in terms of braids. In the other direction, another question, posed by Cameron Gordon, is to provide an explicit geometric description of the passage from a Brunnian braid to a map from $S^{n}$ to $S^{2}$. The geometric differential used in proving Theorem 1.3 may shed some light on this.

8.6. Brunnian braids over $D^{2}$. Recall from the proof of Theorem 1.3 that $G_{n}:=$ $\operatorname{Ker}\left(d_{n}: P_{n+1} \longrightarrow P_{n}\right)$ is the free group of rank $n$ generated by

$$
A_{0, n}, A_{1, n}, \ldots, A_{n-1, n} \text {. }
$$

Let $F\left[S^{1}\right]$ be the Milnor construction of $S^{1}$ with $F\left[S^{1}\right]_{n}=F\left(y_{0}, y_{1}, \ldots, y_{n-1}\right)$ and the faces described in Equation (111). The group homomorphism

$$
\varphi: F\left[S^{1}\right]_{n} \longrightarrow G_{n}
$$

given by

$$
\varphi\left(y_{i}\right)=A_{i, n}
$$

for $0 \leq i \leq n-1$, induces an isomorphism

$$
\phi: N_{n} F\left[S^{1}\right] \stackrel{\cong}{\longrightarrow} G_{n} \cap \bigcap_{i=0}^{n-1} \operatorname{Ker}\left(d_{i}: P_{n+1} \rightarrow P_{n}\right)=\operatorname{Brun}_{n+1}\left(D^{2}\right) .
$$

The Moore chains have been determined in 70. By replacing $y_{i}$ by $A_{i, n}$ under the isomorphism $\phi$, the Brunnian braids $\operatorname{Brun}_{n}\left(D^{2}\right)$ will be described below.

Let $G$ be a group and let $[x, y]=x^{-1} y^{-1} x y$ in $G$. A bracket arrangement of weight $n$ in a group $G$ is a map $\beta^{n}: G^{n} \rightarrow G$ which is defined inductively as follows:

$$
\beta^{1}=\operatorname{id}_{G}, \beta^{2}\left(a_{1}, a_{2}\right)=\left[a_{1}, a_{2}\right]
$$

for any $a_{1}, a_{2} \in G$. Suppose that the bracket arrangements of weight $k$ are defined for $1 \leq k<n$ with $n \geq 3$. A map $\beta^{n}: G^{n} \rightarrow G$ is called a bracket arrangement of weight $n$ if $\beta^{n}$ is the composite

$$
G^{n}=G^{k} \times G^{n-k} \stackrel{\beta^{k} \times \beta^{n-k}}{\longrightarrow} G \times G \stackrel{\beta^{2}}{\longrightarrow} G
$$

for some bracket arrangements $\beta^{k}$ and $\beta^{n-k}$ of weight $k$ and $n-k$, respectively, with $1 \leq k<n$. For instance, if $n=3$, there are two bracket arrangements given by $\left[\left[a_{1}, a_{2}\right], a_{3}\right]$ and $\left[a_{1},\left[a_{2}, a_{3}\right]\right]$. 
Theorem 8.6.1. [70, Theorem 4.4] For each $n$, the Brunnian group $\operatorname{Brun}_{n+1}\left(D^{2}\right)$ is the subgroup of

$$
G_{n}=F\left(A_{0, n}, A_{1, n}, \ldots, A_{n-1, n}\right)
$$

generated by all of the commutators of the form

$$
\left[A_{i_{1}, n}^{\epsilon_{1}}, \ldots, A_{i_{t}, n}^{\epsilon_{t}}\right]
$$

where

(1) $\epsilon= \pm 1$;

(2) $0 \leq i_{s} \leq n-1$;

(3) each integer in $\{0,1, \ldots, n-1\}$ appears as at least one of the integers $i_{s}$;

(4) for each $t \geq n+1,[\cdots]$ runs over all of the commutator bracket arrangements of weight $t$.

A recursive algorithm for finding a free basis for $N_{n} F\left[S^{1}\right] \cong \operatorname{Brun}_{n+1}\left(D^{2}\right)$ was described in [70, Theorem 3.5]. This algorithm has been used in Subsection 7.3 for determining a free basis for $\operatorname{Brun}_{4}\left(D^{2}\right)$. Although this algorithm can eventually give a free basis by finitely many steps, as seen in Subsection 7.3 , the explicit computation for determining a free basis still appears complicated. According to [18, the abelianization $N F\left[S^{1}\right]^{\text {ab }}$ of the Moore chains contains certain homotopy-theoretic information. Also observe that the Artin representation on $F\left[S^{1}\right]$ induces a representation on the Moore chains $N F\left[S^{1}\right]$ and so a linear representation on $N F\left[S^{1}\right]^{\text {ab }}$.

A canonical quotient group $\mathrm{A}_{n+1}\left(D^{2}\right)$ of $\operatorname{Brun}_{n+1}\left(D^{2}\right)$ can be obtained by dividing out the generators

$$
\left[A_{i_{1}, n}^{\epsilon_{1}}, \ldots, A_{i_{t}, n}^{\epsilon_{t}}\right]
$$

in Theorem 8.6.1 that satisfy the following additional condition:

(5) one of the integers $i_{s}$ occurs at least twice.

Recall that the group $\operatorname{Lie}(n)$ consists of the elements of weight $n$ in the Lie algebra $\operatorname{Lie}\left(x_{1}, x_{2}, \cdots, x_{n}\right)$, which is the quotient Lie algebra of the free Lie algebra $L\left(x_{1}, x_{2}, \ldots, x_{n}\right)$ over $\mathbb{Z}$ by the two-sided Lie ideal generated by the Lie elements

$$
\left[\left[\left[x_{i_{1}}, x_{i_{2}}\right], \ldots,\right], x_{i_{t}}\right]
$$

with $i_{l}=i_{k}$ for some $1 \leq l<k \leq t$.

The $S_{n}$-module given by $\operatorname{Lie}(n)$ tensored with the sign representation is the top nonvanishing homology group $H_{n-1}\left(F\left(D^{2}, n\right)\right)$ of the configuration space $F\left(D^{2}, n\right)$; see [16]. Other applications of $\operatorname{Lie}(n)$ can be found in 63] and elsewhere. Then the group $\mathrm{A}_{n+1}\left(D^{2}\right)$ is determined by the following statement, which suggests that there might be further connections between $\operatorname{Lie}(n)$ and links.

Theorem 8.6.2. [70, Theorems 6.7 and 6.14] For each $n$, there is an isomorphism of groups

$$
\mathrm{A}_{n+1}\left(D^{2}\right) \cong \operatorname{Lie}(n)
$$

\section{ACKNOWLEDGMENTS}

The authors would like to thank Professors John Moore, Joan Birman, Mark Mahowald, Cameron Gordon, Joe Neisendorfer and John Harper for helpful discussions. These conversations defined what was most important in this paper. The authors also would like to thank many of their colleagues for their suggestions and 
encouragement on this project, particularly their colleagues from the National University of Singapore and the University of Rochester. In particular, suggestions of Jelena Grbic during her stay in Singapore have been of value to the presentation.

\section{REFERENCES}

[1] E. Artin, Theorie der Zopfe, Hamburg Abh. 4 (1925), 47-72.

[2] E. Artin, Theory of Braids, Ann. of Math. 48 (1947), 101-126. MR0019087 (8:367a)

[3] M.G. Barratt and P. J. Eccles, $\Gamma^{+}$-structures-II: A recognition principle for infinite loop spaces, Topology 13 (1974), 113-126. MR0348738(50:1234b)

[4] D. J. Benson and F. R. Cohen, The mod 2 cohomology of the mapping class group for a surface of genus two, Memoirs Amer. Math. Soc. 443 (1991), 93-104. MR1052554 (91g:57002)

[5] S. Bigelow, Braid groups are linear, J. Amer. Math. Soc. 14 (2001), 471-486. MR1815219 (2002a:20043)

[6] J. S. Birman, Braids, links, and mapping class groups, Annals of Math. Studies 82, Princeton Univ. Press, (1975). MR.0375281 (51:11477)

[7] C.-F. Bödigheimer, F. R. Cohen and M. D. Peim, Mapping class groups and function spaces, Contemp. Math. 271 (2001), 17-39. MR.1831345 (2002f:55036)

[8] R. Bott, Configuration spaces and embedding invariants, Turkish J. Math. 20 (1996), 1-17. MR.1392659 (97k:57008)

[9] R. Bott and A. S. Cattaneo, Integral invariants of 3-manifolds, J. Diff. Geometry 48 (1998), 1-13. MR.1622602 (2000i:58040)

[10] A. K. Bousfield and E. B. Curtis, A spectral sequence for the homotopy of nice spaces, Trans. Amer. Math. Soc. 151 (1970), 457-479. MR0267586 (42:2488)

[11] A. K. Bousfield, E. B. Curtis, D. M. Kan, D. G. Quillen, D. L. Rector and J. W. Schlesinger, The mod-p lower central series and the Adams spectral sequence, Topology 5 (1966), 331-342. MR0199862 (33:8002)

[12] G. E. Bredon, Topology and Geometry, Graduate Texts Math. 139, Springer (Berlin, 1993). MR:1224675 (94d:55001)

[13] G. Carlsson, A simplicial group construction for balanced products, Topology 23 (1985), 85-89. MR0721454 (85e:55019)

[14] A. S. Cattaneo, P. Cotta-Ramusino and R. Longoni, Configuration spaces and Vassiliev classes in any dimension, Algebraic and Geometric Topology 2 (2002), 949-1000. MR1936977 (2004a:57014)

[15] E. Cech, Höherdimensionale homotopiegruppen, In: Verhandlungen des Internationalen Mathematikerkongress, Zürich, 1932, p.203. Orell Füssli (Zürich and Leipzig, 1932).

[16] F. R. Cohen, Homology of $\Omega^{n+1} \Sigma^{n+1} X$ and $C_{n+1}(X), n>0$, Bull. Amer. Math. Soc. 79 (1973), 1236-1241. MR0339176 (49:3939)

[17] F. R. Cohen, On combinatorial group theory in homotopy, Contemp. Math. 188 (1995), 57-63. MR:1349129 (97d:55024)

[18] F. R. Cohen and J. Wu, Braid groups, free groups, and the loop space of the 2-sphere, math.AT/0409307, preprint.

[19] F. R. Cohen, and J. Wu, On braid groups, free groups, and the loop space of the 2-sphere, Proc. Skye Topology Conf., Progress in Math. 215 (2003), 93-105. MR2039761|(2005b:55011)

[20] W. L. Chow, On algebraic braid group, Annals of Math. 49 (1948), 654-658. MR0019088 $(8: 367 \mathrm{~b})$

[21] E. B. Curtis, Simplicial homotopy theory, Advances in Math. 6 (1971), 107-209.

[22] E. B. Curtis and M. Mahowald, The unstable Adams spectral sequence for $S^{3}$, Contemp. Math. 96, Amer. Math. Soc. (Providence RI, 1989), 125-162. MR.1022678(91b:55012)

[23] M. Davis, T. Januszkiewicz and R. Scott, Nonpositive curvature of blow-ups, Selecta Math. 4 (1998), 491-547. MR.1668119 (2001f:53078)

[24] H. Debrunner, Links of Brunnian type, Duke Math. J. 28 (1961), 17-23. MR0137106 (25:562)

[25] S. Devadoss, Tessellations of moduli spaces and the mosaic operad, in Homotopy invariant algebraic structures, Contemp. Math. 239 (1998), 91-114. MR.1718078 (2000i:18014)

[26] E. Fadell and L. Neuwirth, Configuration spaces, Math. Scand. 10 (1962), 111-118. MR0141126 (25:4537)

[27] E. Fadell and J. Van Buskirk, The braid groups of $E^{2}$ and $S^{2}$, Duke Math. J. 29 (1962), 243-258. MR0141128 (25:4539) 
[28] M. Falk and R. Randell, The lower central series of a fibre-type arrangement, Invent. Math. 82 (1985), 77-88. MR0808110 (87c:32015b)

[29] E. M. Feichtner, The integral cohomology algebra of ordered configuration spaces of spheres, Documenta Math. 5 (2000), 115-139. MR1752611 (2002b:55031)

[30] Z. Feidorowicz and J-L. Loday, Crossed simplicial groups and their associated homology, Trans. Amer. Math. Soc. 326 (1991), 57-87. MR0998125 (91j:18018)

[31] W. Fulton and R. MacPherson, Compactification of configuration spaces, Annals of Math. 139 (1994), 183-225. MR 1259368 (95j:14002)

[32] R. Gillette and Van Buskirk, The word problem and its consequences for the braid groups and mapping class groups of the 2-sphere, Trans. Amer. Math. Soc. 131 (1968), 277-296. MR0231894 (38:221)

[33] P. Hall, A contribution to the theory of groups of prime power order, Proc. London. Math. Soc. 2 (1936), 29-95.

[34] R. Hartshorne, Algebraic Geometry, Graduate Texts Math. 52, Springer (New York, 1977). MR.0463157 (57:3116)

[35] M.W. Hirsch, Differential Topology, Graduate Texts Math. 33, Springer (New York, 1976). MR0448362 (56:6669)

[36] H. Hopf, Über die Topologie der Grunppenmannigfaltigkeiten und ihre Verallgemeinerungen, Annals of Math. (2) 42 (1941), 22-52. MR0004784 (3:61b)

[37] W. Hurewicz, Beiträge zur Topologie der deformationen $I-I V$, Nederl. Akad. Wetensch. Proc. Ser. A 38 (1936), 117-126, 215-224.

[38] I. M. James, On the suspension sequence, Annals of Math. 65 (1957), 74-107. MR0083124 $(18: 662 \mathrm{e})$

[39] D. L. Johnson, Towards a characterization of smooth braids, Math. Proc. Cambridge Philos. Soc. 92 (1982), 425-427. MR0677467 (84c:20048)

[40] D. Kan, A combinatorial definition of homotopy groups, Annals of Math. 67 (1958), 288-312. MR0111032 (22:1897)

[41] S. Keel, Intersection theory of moduli space of stable $N$-pointed curves of genus zero, Trans. Amer. Math. Soc. 330 (1992), 545-574. MR1034665 (92f:14003)

[42] F. Kirwan, Complex Algebraic Curves, LMS Student Texts 23, Cambridge Univ. Press (Cambridge, 1992). MR1159092 (93j:14025)

[43] T. Kohno, Série de Poincaré-Koszul associée aux groupes de tresses pures, Invent. Math. 82 (1985), 57-75. MR0808109 (87c:32015a)

[44] T. Kohno, Vassiliev invariants and de Rham complex on the space of knots, in: Symplectic Geometry and Quantization, Contemp. Math. 179, Amer. Math. Soc. (Providence RI, 1994), 123-138. MR 1319605 (96g:57010)

[45] T. Kohno, Elliptic $K Z$ system, braid groups of the torus and Vassiliev invariants, Topology and its Applications, 78 (1997), 79-94. MR1465026 (2000c:57023)

[46] T. Kohno, Loop spaces of configuration spaces and finite type invariants, Geom. Topol. Monogr. 4 (2002), 143-160. MR2002608 (2004g:55014)

[47] D. Krammer, Braid groups are linear, Annals of Math. 155 (2002), 131-156. MR1888796 (2003c:20040)

[48] H. Levinson, Decomposable braids and linkages, Trans. Amer. Math. Soc. 178 (1973), 111126. MR0324684 (48:3034)

[49] C. Liang and K. Mislow, On Borromean links, J. Math. Chem. 16 (1994), 27-35. MR.1304179 $(95 \mathrm{j}: 57008)$

[50] W. Magnus, A. Karrass and D. Solitar, Combinatorial Group Theory, 2nd ed., Dover (New York, 1976). MR0422434 (54:10423)

[51] B. Mangum and T. Stanford, Brunnian links are determined by their complements, Algebraic and Geometric Topology 1 (2001), 143-152. MR1823496 (2002e:57009)

[52] J. P. May, Simplicial Objects in Algebraic Topology, Math. Studies 11, van Nostrand (Princeton NJ, 1967). MR0222892 (36:5942)

[53] J. P. May, The Geometry of Iterated Loop Spaces, Lect. Notes in Math. 271, Springer (1972). MR0420610 (54:8623b)

[54] J. Milnor, The geometric realization of a semi-simplicial complex, Annals of Math. 65 (1957), 357-362. MR0084138(18:815d)

[55] J. Milnor, On the construction $F[K]$, Algebraic Topology - A Student Guide, by J. F. Adams, Cambridge Univ. Press, 119-136. 
[56] J. C. Moore, Homotopie des complexes monöidéaux, Séminaire Henri Cartan (1954-55).

[57] H. Poincaré, Analysis situs, J. École Polytech. (2) 1 (1895), 1-121.

[58] D. E. Penney, Generalized Brunnian links, Duke Math. J. 36 (1969), 31-32. MR0238302 (38:6578)

[59] D. G. Quillen, Homotopical algebra, Lect. Notes in Math. 43, Springer-Verlag (Berlin, 1967). MR0223432 (36:6480)

[60] D. Rolfsen, Knots and Links, Math. Lect. Series 7, Publish or Perish (Berkeley, 1976). MR0515288 (58:24236)

[61] T. Sato, On the group of morphisms of coalgebras, Ph.D. Thesis, Univ. Rochester, 2000.

[62] P. S. Selick, A decomposition of $\pi_{*}\left(S^{2 p+1} ; p\right)$, Topology 20 (1981), 175-177. MR0605656 (82c:55017)

[63] P. Selick and J. Wu, On natural decompositions of loop suspensions and natural coalgebra decompositions of tensor algebras, Memoirs Amer. Math. Soc. 148 no. 701, (2000). MR1706247 (2001c:55006)

[64] J-P. Serre, Homologie singulière des espaces fibrés. Applications, Annals of Math. (2) 54 (1951), 425-505. MR0045386 (13:574g)

[65] J. H. Smith, Simplicial group models for $\Omega^{n} \Sigma^{n} X$, Israel J. Math. 66 (1989), 330-350. MR1017171 (91e:55014)

[66] E. H. Spanier, Algebraic Topology, McGraw-Hill (New York, 1966). MR0210112 (35:1007)

[67] H. Toda, Composition methods in homotopy groups of spheres, Annals of Math. Studies 49, Princeton Univ. Press (Princeton, 1962). MR0143217 (26:777)

[68] K. Whittlesey, Normal all pseudo-Anosov subgroups of mapping class groups, Geometry and Topology 4 (2000), 293-307. MR1786168(2001j:57022)

[69] J. Wu, On fibrewise simplicial monoids and Milnor-Carlsson's constructions, Topology 37 (1998), 1113-1134. MR1650351 (99h:55030)

[70] J. Wu, Combinatorial descriptions of the homotopy groups of certain spaces, Math. Proc. Camb. Philos. Soc. 130 (2001), 489-513. MR1816806 (2003e:55014)

[71] J. Wu, A braided simplicial group, Proc. London Math. Soc. 84 (2002), 645-662. MR1888426 (2003e:20041)

[72] M.A. Xicoténcatl, Orbit Configuration spaces, infinitesimal braid relations in homology and equivariant loop spaces, Ph.D. Thesis, Univ. Rochester (1997).

[73] M.A. Xicoténcatl, The Lie algebra of the pure braid group, Bol. Soc. Mat. Mexicana 6 (2000), 55-62. MR.1768508 (2001d:20036)

Department of Mathematics, National University of Singapore, Kent Ridge 117543, SINGAPORE

E-mail address: berrick@math.nus.edu.sg

Department of Mathematics, University of Rochester, Rochester, New York 14627

E-mail address: cohf@math.rochester.edu

Department of Mathematics, National University of Singapore, Kent Ridge 117543, SINGAPORE

E-mail address: matwyl@nus.edu.sg

Department of Mathematics, National University of Singapore, Kent Ridge 117543 , SINGAPORE

E-mail address: matwuj@nus.edu.sg 